\title{
Kinase-Inhibitory Nucleoside Derivatives from the Pacific Bryozoan Nelliella nelliiformis.
}

Joe Bracegirdle, Dennis P. Gordon, Joanne E. Harvey, Robert A. Keyzers

Figure S1: GCMS analysis of 1

Table S1: ${ }^{13} \mathrm{C}(150 \mathrm{MHz})$ and ${ }^{1} \mathrm{H}(600 \mathrm{MHz}) \mathrm{NMR}$ data of 1

Table S2: Comparison of NMR shifts for natural and synthetic 1

Figure S2: ${ }^{1} \mathrm{H}$ NMR Spectrum of $\mathbf{1} \ldots \ldots \ldots$

Figure S3: ${ }^{13} \mathrm{C}$ NMR Spectrum of 1

Figure S4: COSY NMR Spectrum of 1

Figure S5: HSQC NMR Spectrum of 1

Figure S6: HMBC NMR Spectrum of 1

Figure S7: ROESY NMR Spectrum of 1

Figure S8: MS Spectrum of 1

Figure S9: MS/MS Spectrum of 1

Figure S10: IR Spectrum of 1

Figure S11: UV/Vis Spectrum of 1

Table S3: ${ }^{13} \mathrm{C}(150 \mathrm{MHz})$ and ${ }^{1} \mathrm{H}(600 \mathrm{MHz}) \mathrm{NMR}$ data of $2 \ldots 13$

Table S4: Comparison of NMR shifts for natural and synthetic $2 \ldots 14$

Figure S12: ${ }^{1} \mathrm{H}$ NMR Spectrum of $2 \ldots 15$

Figure S13: ${ }^{13} \mathrm{C}$ NMR Spectrum of $2 \ldots 16$

Figure S14: COSY NMR Spectrum of 2

Figure S15: HSQC NMR Spectrum of 2

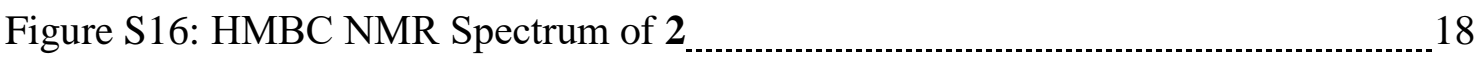

Figure S17: MS Spectrum of 2

Figure S18: MS/MS Spectrum of 2

Figure S19: IR Spectrum of 2

Figure S20: UV/Vis Spectrum of 2

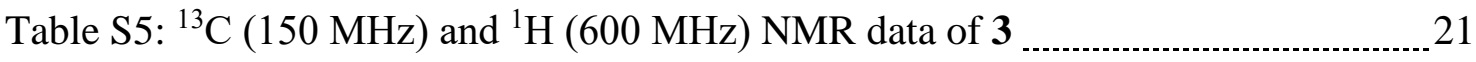

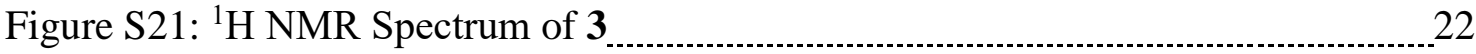


Figure S22: ${ }^{13} \mathrm{C}$ NMR Spectrum of 3

Figure S23: COSY NMR Spectrum of 3

Figure S24: HSQC NMR Spectrum of 3

Figure S25: HMBC NMR Spectrum of $\mathbf{3} \ldots \ldots \ldots$

Figure S26: MS Spectrum of 3

Figure S27: MS/MS Spectrum of 3

Figure S28: IR Spectrum of $\mathbf{3} \ldots$

Figure S29: UV/Vis Spectrum of 3

Table S6: ${ }^{13} \mathrm{C}(150 \mathrm{MHz})$ and ${ }^{1} \mathrm{H}(600 \mathrm{MHz})$ NMR data of 4

Figure S30: ${ }^{1} \mathrm{H}$ NMR Spectrum of $4 \ldots \ldots$

Figure S31: ${ }^{13} \mathrm{C}$ NMR Spectrum of 4

Figure S32: COSY NMR Spectrum of 4

Figure S33: HSQC NMR Spectrum of 4

Figure S34: HMBC NMR Spectrum of 4

Figure S35: MS Spectrum of 4 32

Figure S36: MS/MS Spectrum of 4 33

Figure S37: IR Spectrum of 4

Figure S38: UV/Vis Spectrum of 4

Table S7: ${ }^{13} \mathrm{C}(150 \mathrm{MHz})$ and ${ }^{1} \mathrm{H}(600 \mathrm{MHz})$ NMR data of 5

Figure S39: ${ }^{1} \mathrm{H}$ NMR Spectrum of 5

Figure S40: ${ }^{13} \mathrm{C}$ NMR Spectrum of 5

Figure S41: COSY NMR Spectrum of 5

Figure S42: HSQC NMR Spectrum of 5

Figure S43: HMBC NMR Spectrum of 5

Figure S44: MS Spectrum of $\mathbf{5}$

Figure S45: MS/MS Spectrum of 5

Figure S46: IR Spectrum of 5

Figure S47: UV/Vis Spectrum of 5 
Table S8: ${ }^{13} \mathrm{C}(150 \mathrm{MHz})$ and ${ }^{1} \mathrm{H}(600 \mathrm{MHz})$ NMR data of 6 42

Figure S48: ${ }^{1} \mathrm{H}$ NMR Spectrum of 6

Figure S49: ${ }^{13} \mathrm{C}$ NMR Spectrum of 6

Figure S50: COSY NMR Spectrum of 6

Figure S51: HSQC NMR Spectrum of 6

Figure S52: HMBC NMR Spectrum of 6 46

Figure S53: MS Spectrum of 6

Figure S54: MS/MS Spectrum of 6

Figure S55: IR Spectrum of 6

Figure S56: UV/Vis Spectrum of 6

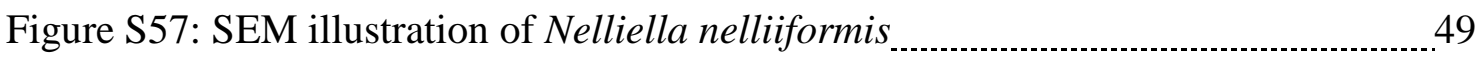

Table S9: Kinome inhibitory screen data for 1 
Figure S1. GCMS Retention Time Comparisons of Furanose Sugars. Left: achiral (Restek RXI-5Sil MS), right: chiral (Agilent Chira-Sil-L-Val).
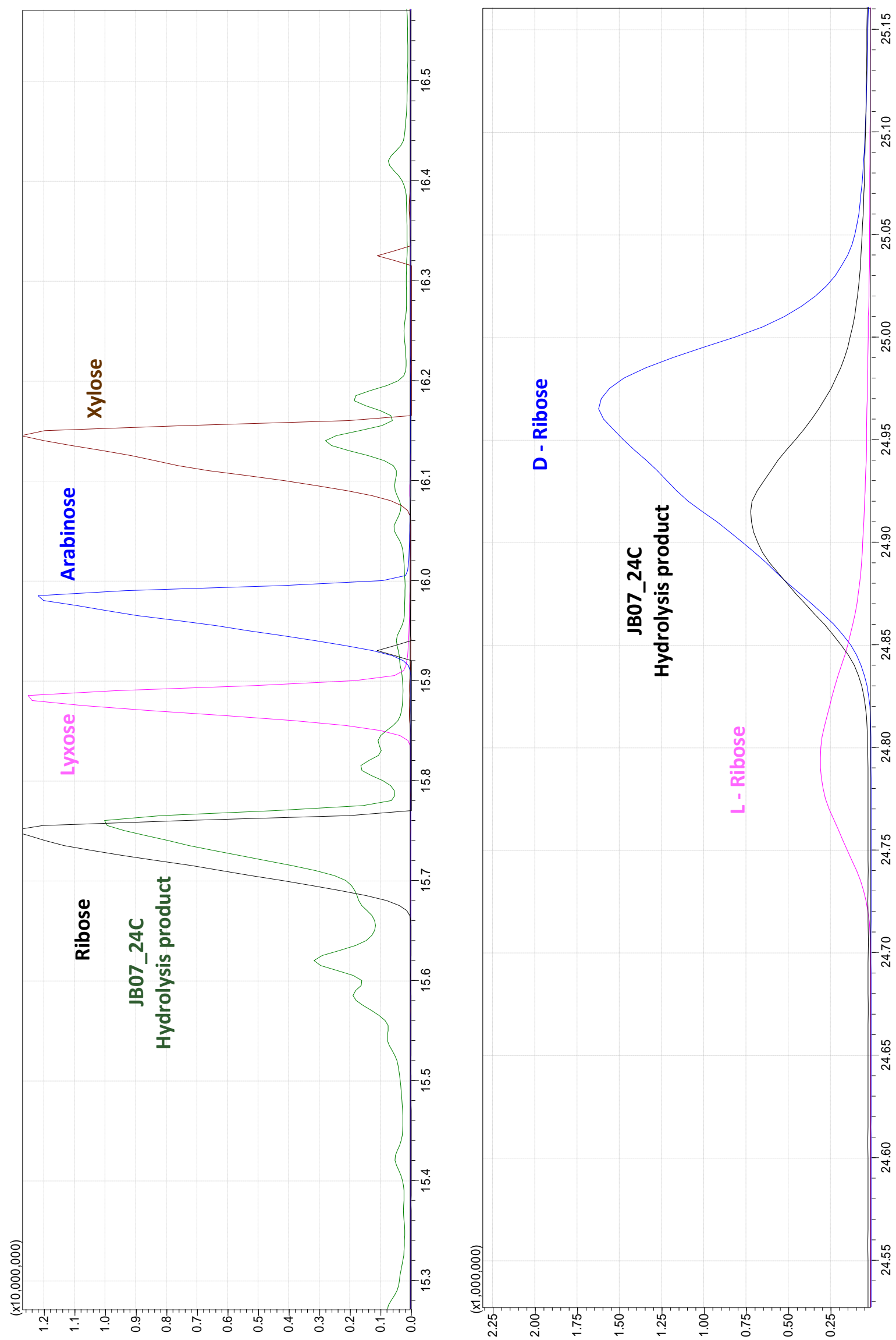
Table S1: ${ }^{13} \mathrm{C}(150 \mathrm{MHz})$ and ${ }^{1} \mathrm{H}(600 \mathrm{MHz}) \mathrm{NMR}$ data for Nellielloside A (1) in DMSO-d 6

\begin{tabular}{|c|c|c|c|c|}
\hline & ${ }^{13} \mathrm{C}(\delta)$ & 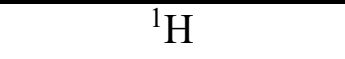 & COSY & HMBC \\
\hline position & & $(\delta$, mult., $J$ in $H z)$ & & \\
\hline 2 & 152.7 & $8.13(\mathrm{~s})$ & & 4,6 \\
\hline 4 & 149.4 & & & \\
\hline 5 & 119.1 & & & \\
\hline 6 & 156.1 & & & \\
\hline $\mathrm{NH}_{2}-6$ & & $7.28(\mathrm{~s})$ & & 5 \\
\hline 8 & 139.4 & $8.25(\mathrm{~s})$ & & 4,5 \\
\hline $1^{\prime}$ & 87.6 & $5.92(\mathrm{~d}, 5.2)$ & $2^{\prime}$ & $4,8,2^{\prime}, 3^{\prime}$ \\
\hline $2^{\prime}$ & 73.1 & $4.66(t, 5.2)$ & $1^{\prime}, 3^{\prime}$ & $3^{\prime}$ \\
\hline $3^{\prime}$ & 70.3 & $4.32(\mathrm{t}, 4.9)$ & $2^{\prime}, 4^{\prime}$ & $1^{\prime}, 4^{\prime}$ \\
\hline $4^{\prime}$ & 81.8 & $4.18(\mathrm{~m})$ & $3^{\prime}, 5^{\prime}$ & $1^{\prime}, 5^{\prime}$ \\
\hline \multirow[t]{2}{*}{$5^{\prime}$} & 63.4 & $4.50(\mathrm{dd}, 12.0,3.7)$ & $4^{\prime}$ & $3^{\prime}, 6^{\prime \prime}$ \\
\hline & & $4.36(\mathrm{dd}, 12.0,5.7)$ & $4^{\prime}$ & $3^{\prime}, 4^{\prime}, 6^{\prime \prime}$ \\
\hline NH-1" & & $11.94(\mathrm{~s})$ & 3", 4", 5" & \\
\hline $2^{\prime \prime}$ & 121.5 & & & \\
\hline $3^{\prime \prime}$ & 115.4 & $6.81(\mathrm{~m})$ & NH-1", 4", 5" & \\
\hline $4 "$ & 109.7 & $6.17(\mathrm{~m})$ & NH-1", 3", 5" & \\
\hline $5^{\prime \prime}$ & 124.5 & $7.03(\mathrm{~m})$ & NH-1", 3", 4" & 2", 3", 4" \\
\hline $6^{\prime \prime}$ & 160.1 & & & \\
\hline
\end{tabular}

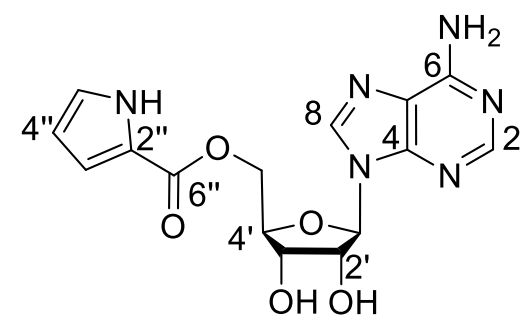

1 
Table S2: Comparison of ${ }^{13} \mathrm{C}(150 \mathrm{MHz})$ and ${ }^{1} \mathrm{H}(600 \mathrm{MHz}) \mathrm{NMR}$ shifts for natural and synthetic 1 in DMSO-d 6

\begin{tabular}{|c|c|c|c|c|}
\hline \multirow[b]{2}{*}{ Position } & \multicolumn{2}{|c|}{ Natural Sample } & \multicolumn{2}{|c|}{ Synthetic Sample } \\
\hline & ${ }^{13} \mathrm{C}$ & ${ }^{1} \mathrm{H}$ & ${ }^{13} \mathrm{C}$ & ${ }^{1} \mathrm{H}$ \\
\hline 2 & 152.7 & 8.13 & 152.8 & 8.14 \\
\hline 4 & 149.4 & & 149.4 & \\
\hline 5 & 119.1 & & 119.2 & \\
\hline 6 & 156.1 & & 156.1 & \\
\hline $\mathrm{NH}_{2}-6$ & & 7.28 & & 7.28 \\
\hline 8 & 139.4 & 8.25 & 139.5 & 8.25 \\
\hline $1^{\prime}$ & 87.6 & 5.92 & 87.6 & 5.93 \\
\hline $2^{\prime}$ & 73.1 & 4.66 & 73.1 & 4.67 \\
\hline $3^{\prime}$ & 70.3 & 4.32 & 70.4 & 4.33 \\
\hline $4^{\prime}$ & 81.8 & 4.18 & 81.9 & 4.18 \\
\hline \multirow[t]{2}{*}{$5^{\prime}$} & 63.4 & 4.50 & 63.4 & 4.51 \\
\hline & & 4.36 & & 4.37 \\
\hline NH-1" & & 11.94 & & 11.89 \\
\hline $2 "$ & 121.5 & & 121.5 & \\
\hline $3 "$ & 115.4 & 6.81 & 115.5 & 6.81 \\
\hline $4 "$ & 109.7 & 6.17 & 109.8 & 6.18 \\
\hline $5^{\prime \prime}$ & 124.5 & 7.03 & 124.5 & 7.04 \\
\hline $6 "$ & 160.1 & & 160.2 & \\
\hline
\end{tabular}


Figure S2: ${ }^{1} \mathrm{H}$ NMR Spectrum (600 MHz, DMSO-d $\left.\mathrm{d}_{6}\right)$ of $\mathbf{1}$

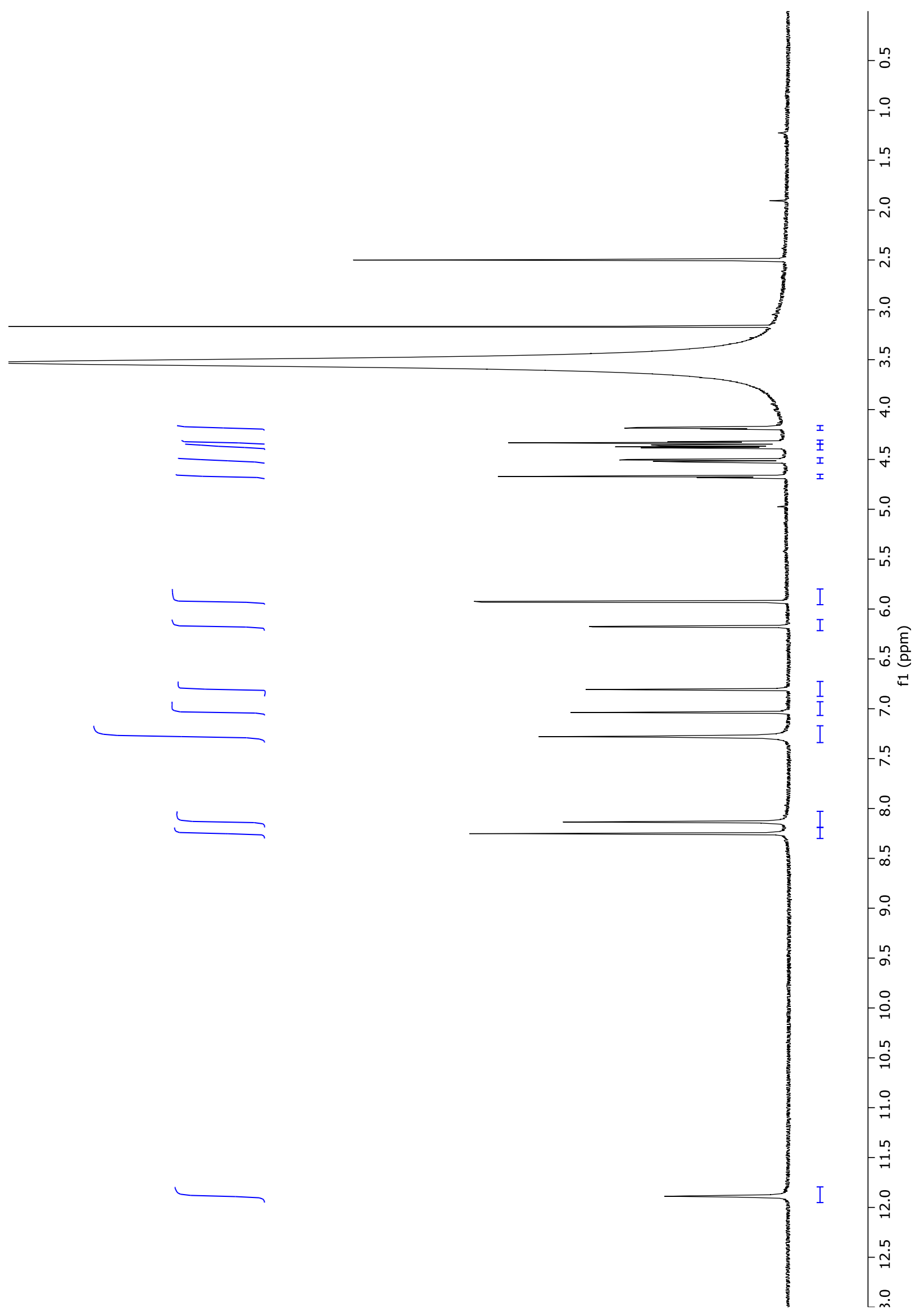




$$
1
$$


Figure S4: COSY Spectrum $\left(600 \mathrm{MHz}, \mathrm{DMSO}-\mathrm{d}_{6}\right)$ of $\mathbf{1}$

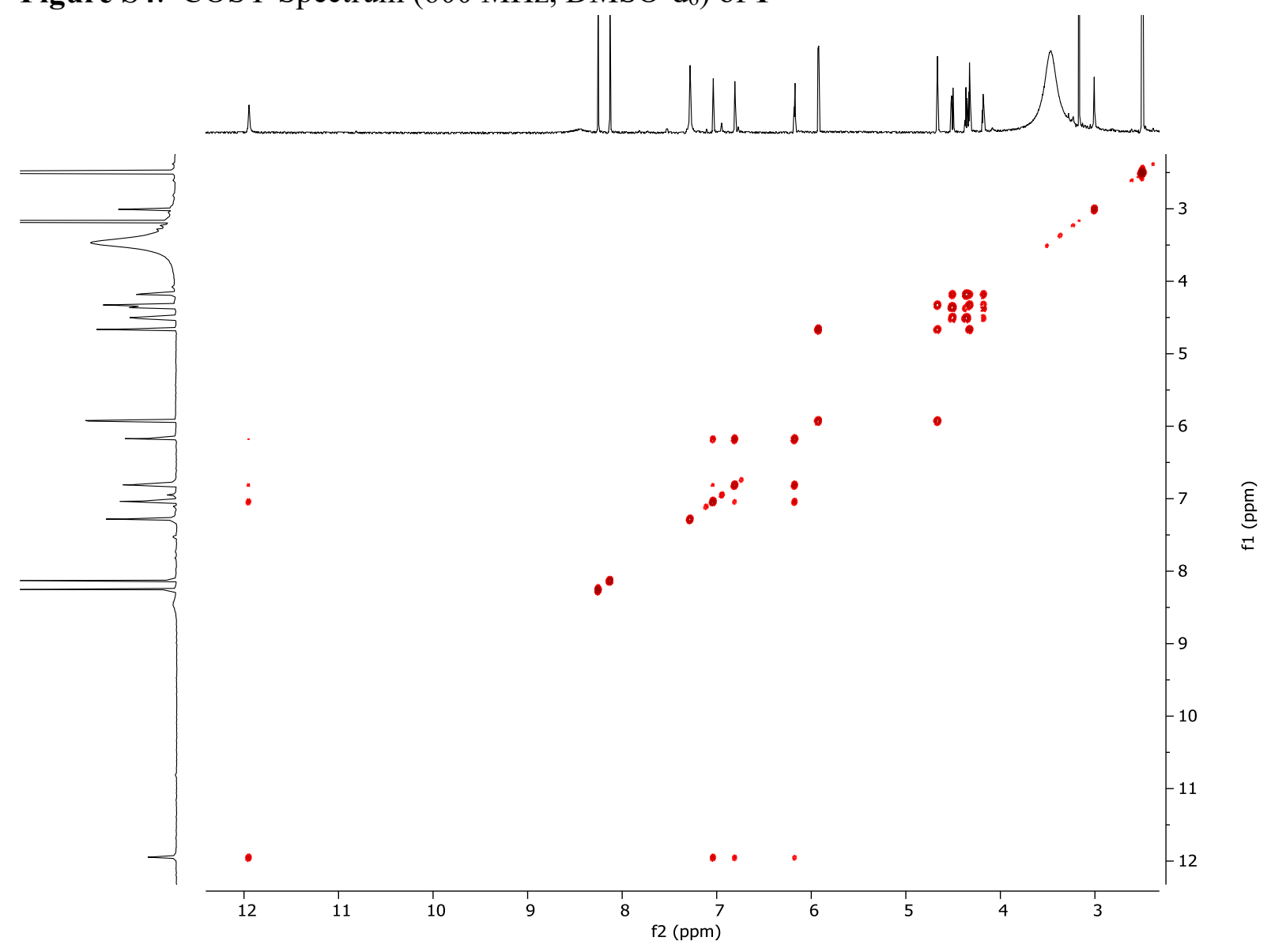

Figure S5: HSQC Spectrum (600 MHz, DMSO-d 6 ) of 1

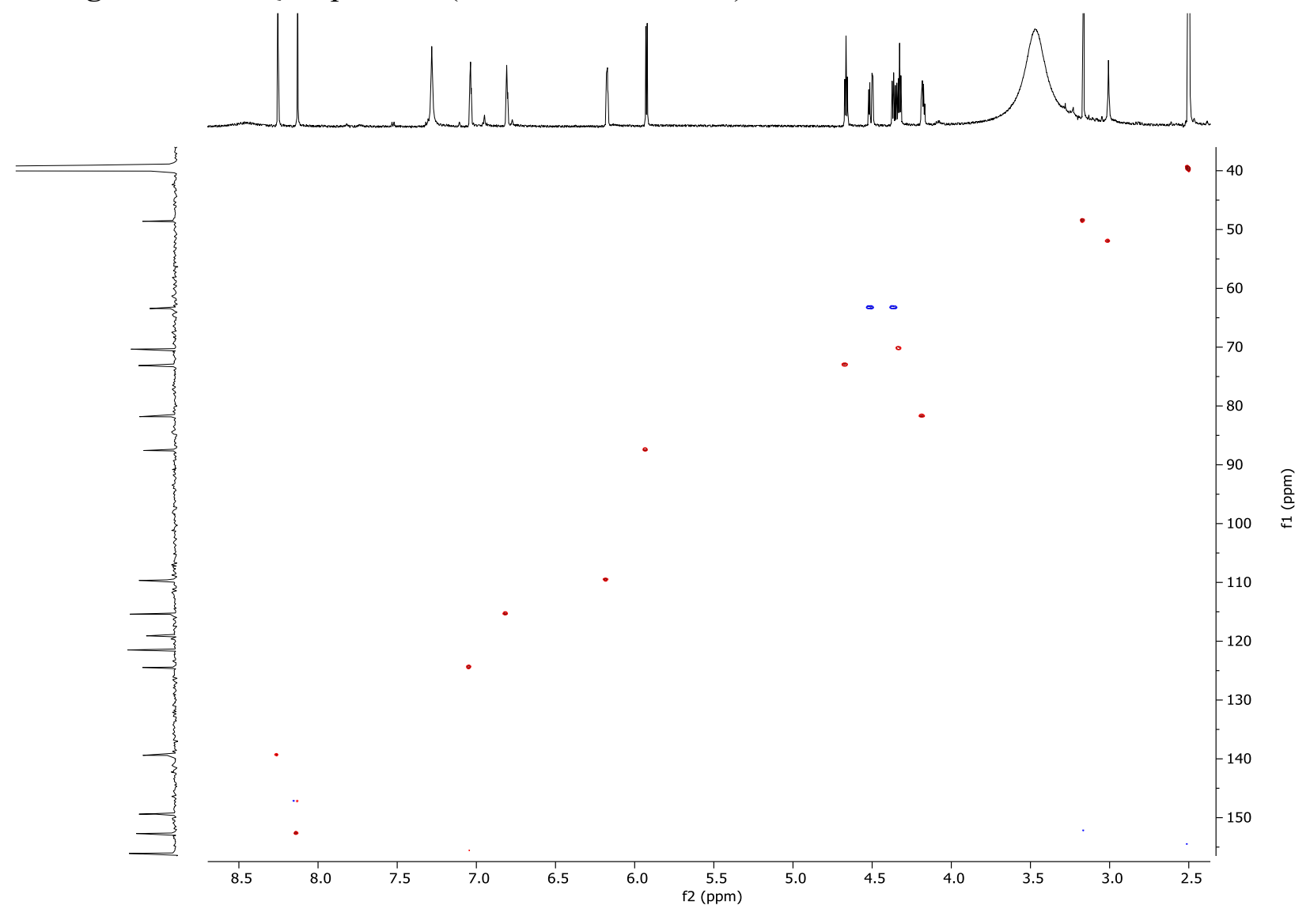


Figure S6: HMBC Spectrum $\left(600 \mathrm{MHz}, \mathrm{DMSO}-\mathrm{d}_{6}\right)$ of 1

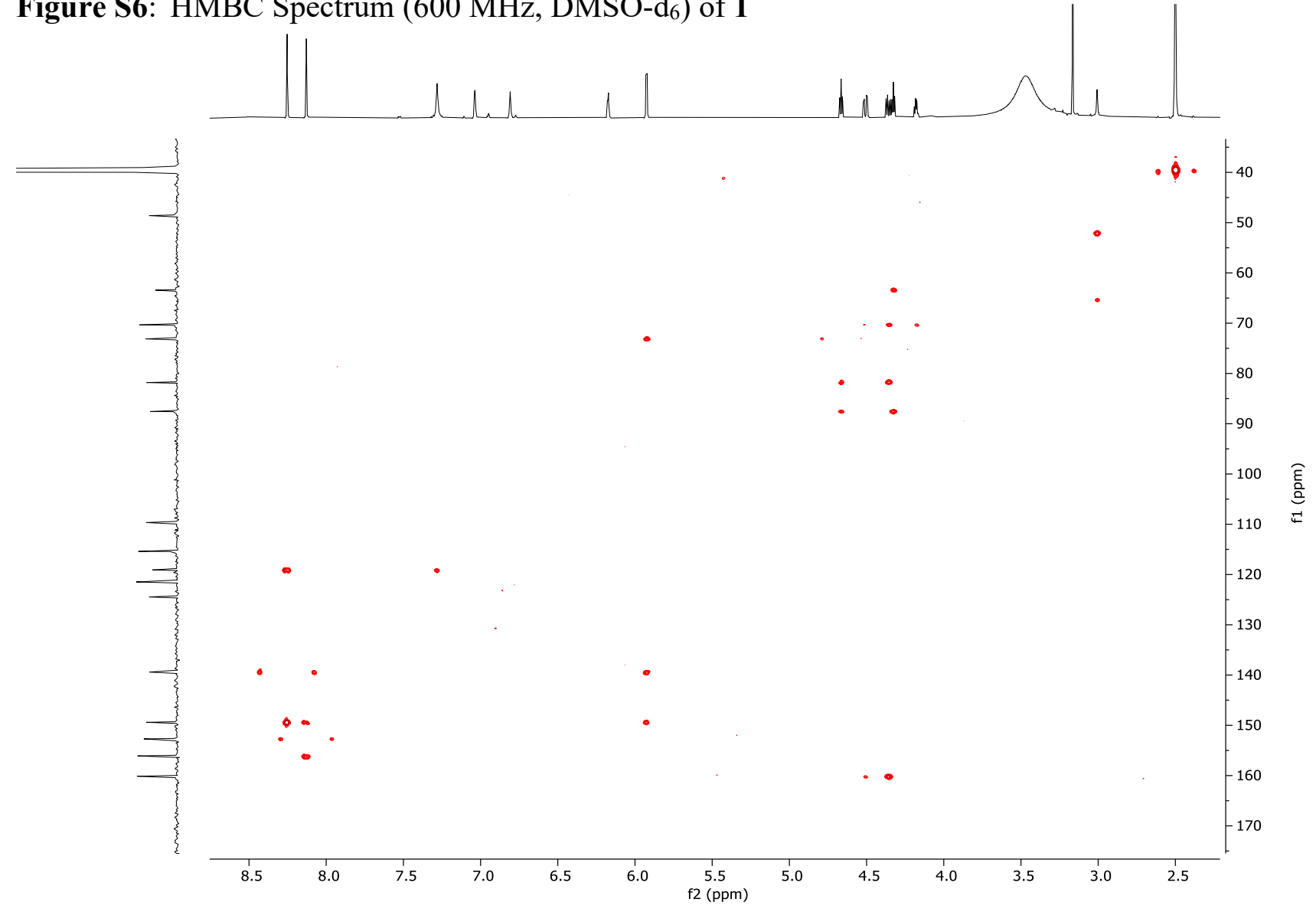

Figure S7: ROESY Spectrum (600 MHz, DMSO-d $)$ of 1

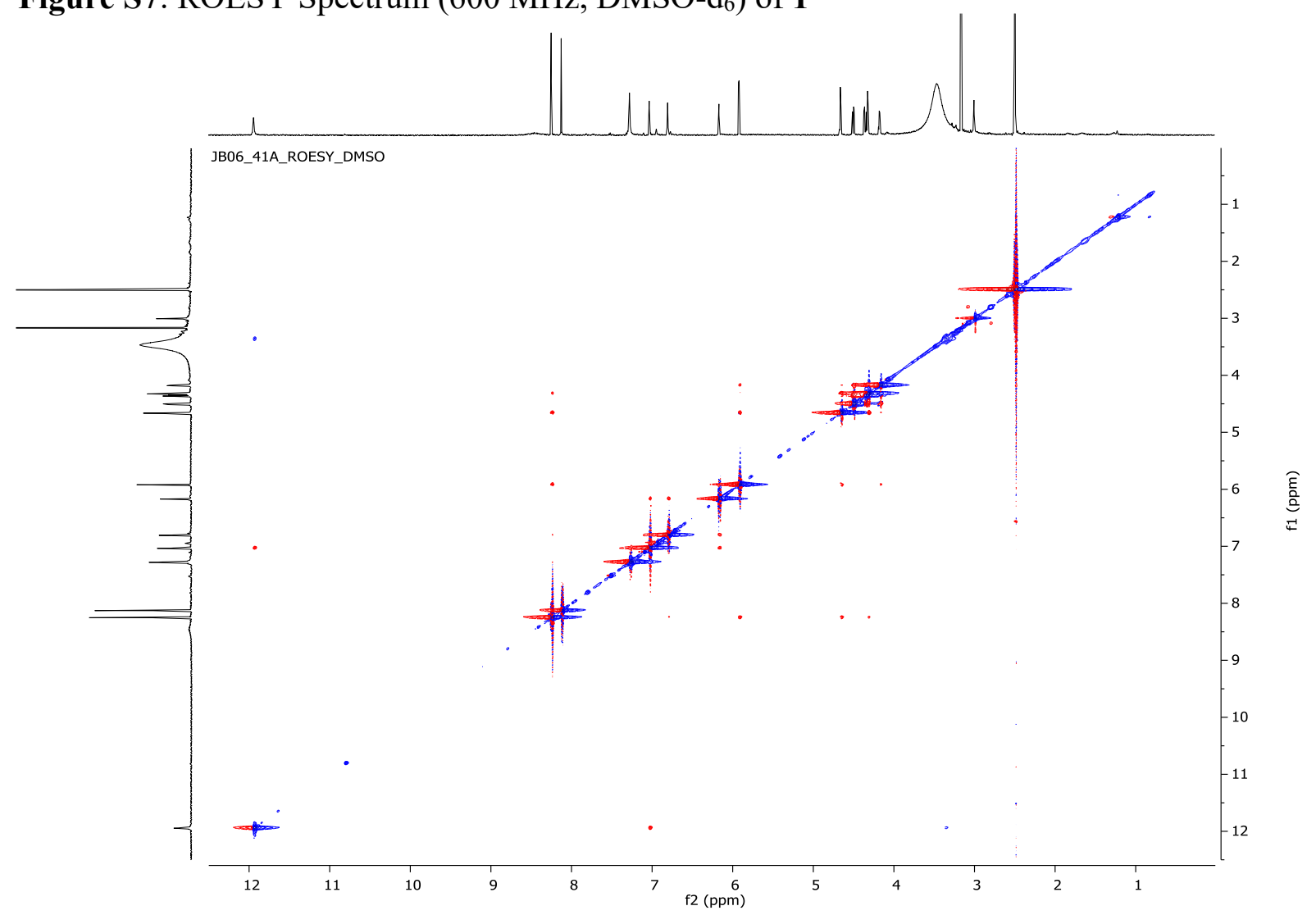


Figure S8: (+)-HRESIMS spectrum of 1

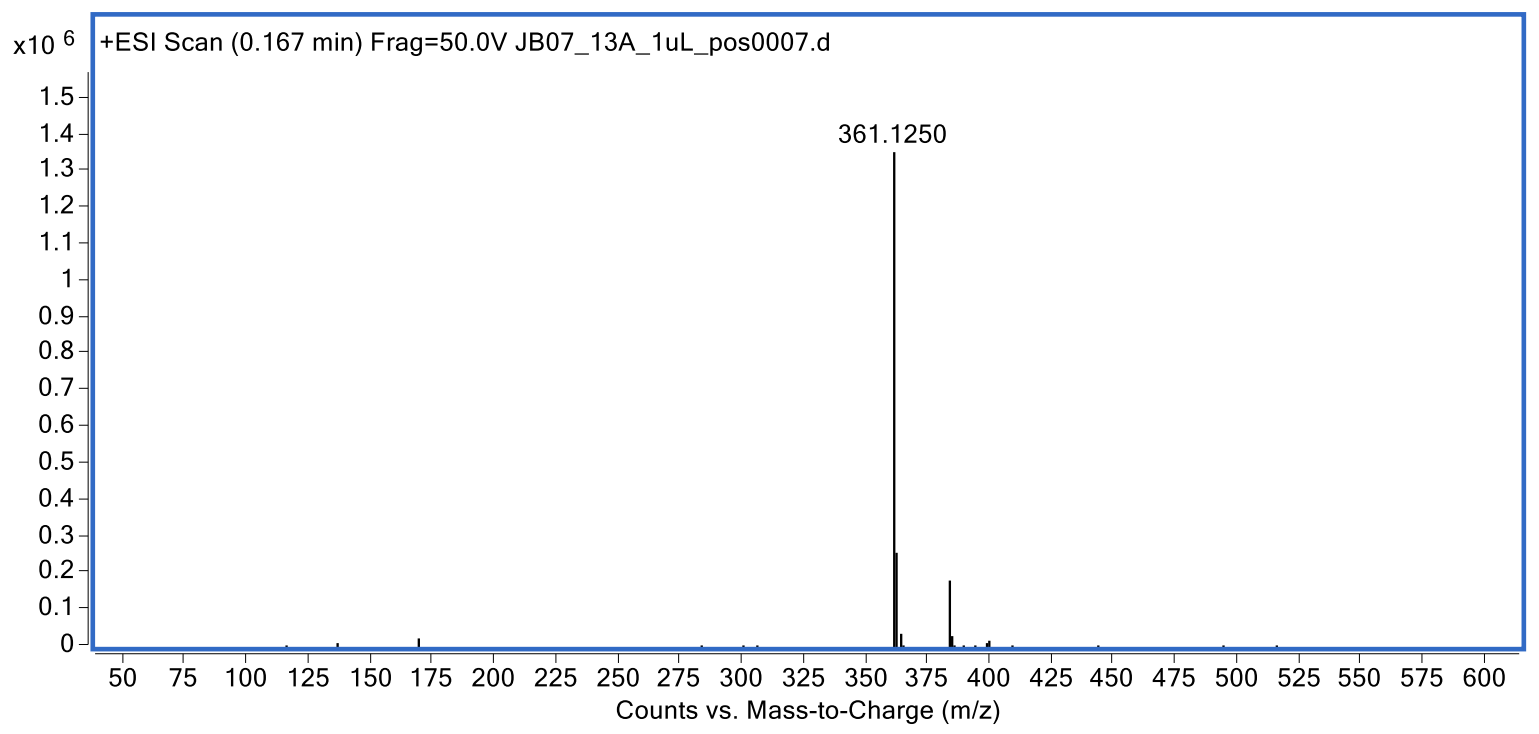

Figure S9: (+)-HRESI-MS/MS spectrum of 1

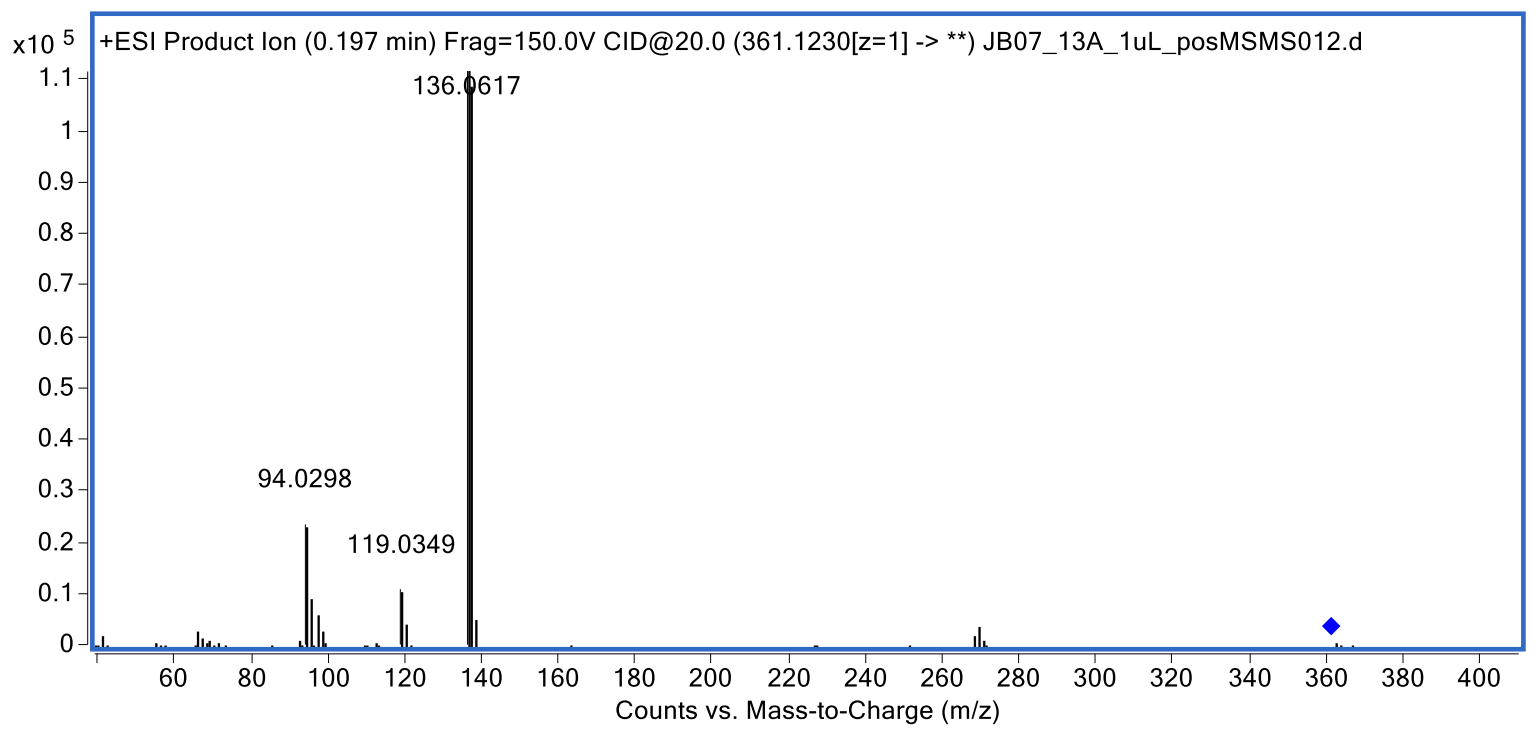


Figure S10: IR spectrum of 1

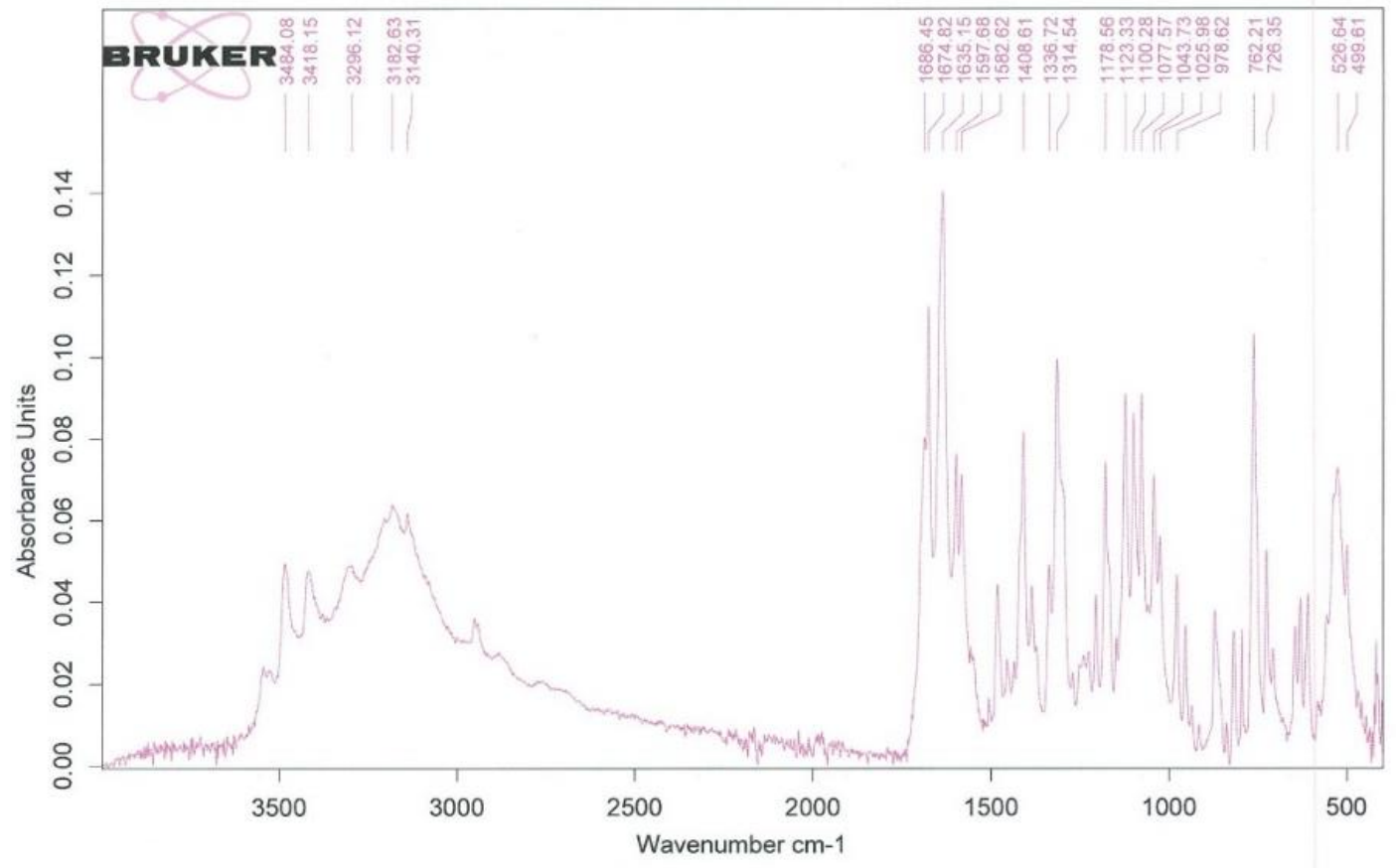

Figure S11: UV/Vis spectrum of 1

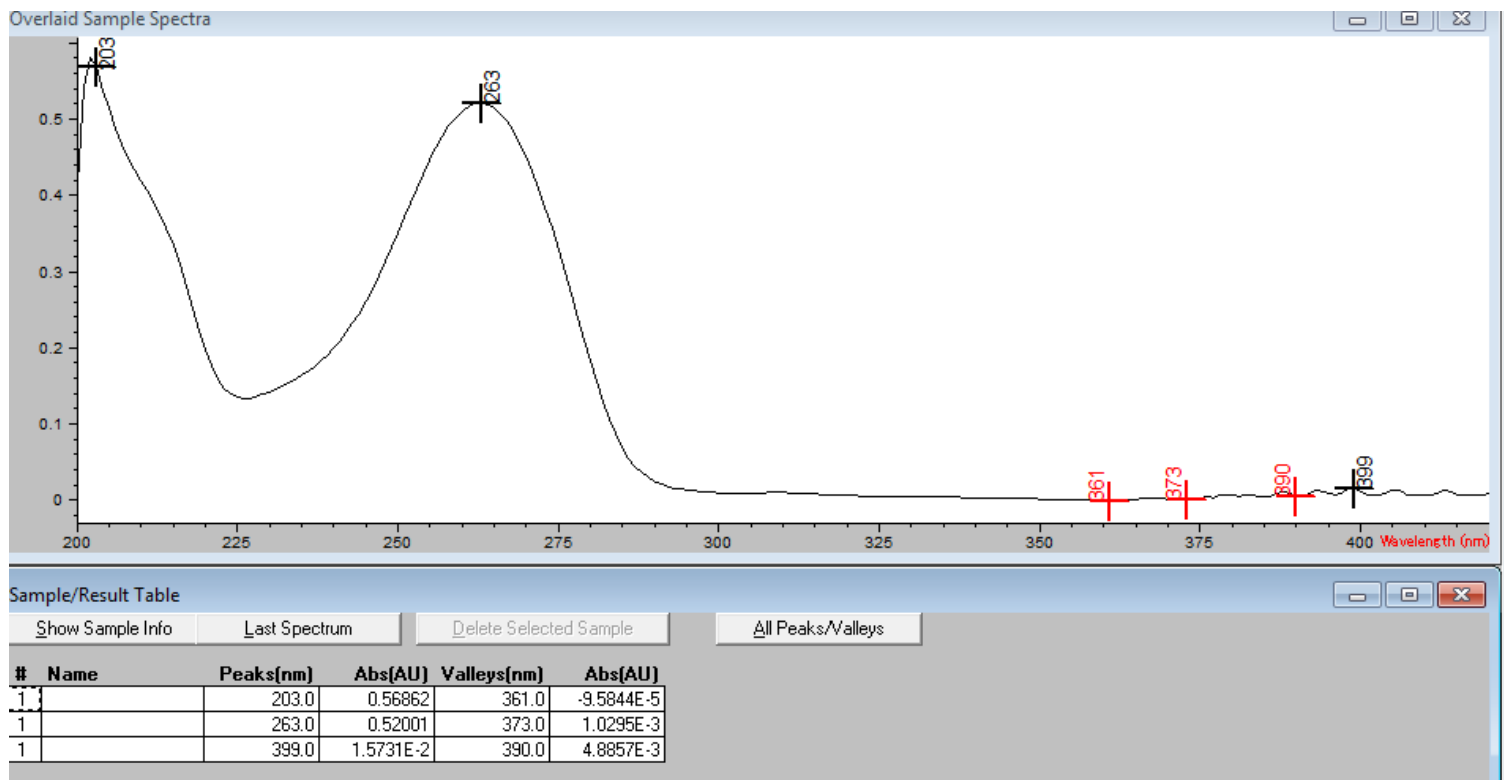


Table S3: ${ }^{13} \mathrm{C}(150 \mathrm{MHz})$ and ${ }^{1} \mathrm{H}(600 \mathrm{MHz})$ NMR data for Nellielloside B (2) in DMSO- $\mathrm{d}_{6}$

\begin{tabular}{|c|c|c|c|c|}
\hline position & ${ }^{13} \mathrm{C}(\delta)$ & $\begin{array}{c}{ }^{1} \mathrm{H} \\
(\delta, \text { mult., } J \text { in } H z)\end{array}$ & COSY & HMBC \\
\hline 2 & 150.5 & $7.92(\mathrm{~s})$ & & 4,6 \\
\hline 4 & 148.8 & & & \\
\hline 5 & 124.5 & & & \\
\hline 6 & 162.5 & & & \\
\hline 8 & 136.8 & $7.99(\mathrm{~s})$ & & 4,5 \\
\hline $1^{\prime}$ & 87.6 & $5.85(\mathrm{~d}, 5.1)$ & $2^{\prime}$ & $4,8,2^{\prime}$ \\
\hline $2^{\prime}$ & 73.2 & $4.62(\mathrm{t}, 5.1)$ & $1^{\prime}, 3^{\prime}$ & $1^{\prime}, 4^{\prime}$ \\
\hline $3^{\prime}$ & 70.3 & $4.29(\mathrm{t}, 5.0)$ & $2^{\prime}, 4^{\prime}$ & $1^{\prime}, 5^{\prime}$ \\
\hline $4^{\prime}$ & 81.6 & $4.16(\mathrm{~m})$ & $3^{\prime}, 5^{\prime}$ & $3^{\prime}$ \\
\hline \multirow[t]{2}{*}{$5^{\prime}$} & 63.5 & $4.48(\mathrm{dd}, 12.0,3.7)$ & $4^{\prime}$ & $3^{\prime}, 6^{\prime \prime}$ \\
\hline & & $4.35(\mathrm{dd}, 12.0,5.7)$ & $4^{\prime}$ & $3^{\prime}, 4^{\prime}, 6^{\prime \prime}$ \\
\hline NH-1" & & $11.96(\mathrm{~s})$ & 3", 4", 5" & \\
\hline $2 "$ & 121.4 & & & \\
\hline $3^{\prime \prime}$ & 115.4 & $6.82(\mathrm{~m})$ & NH-1", 4", 5" & $2{ }^{\prime \prime}, 4 ’$ \\
\hline $4 "$ & 109.7 & $6.18(\mathrm{~m})$ & NH-1", 3", 5" & \\
\hline $5^{\prime \prime}$ & 124.4 & $7.04(\mathrm{~m})$ & NH-1", 3", 4" & $2^{\prime \prime}, 3 ", 4 "$ \\
\hline $6^{\prime \prime}$ & 160.1 & & & \\
\hline
\end{tabular}

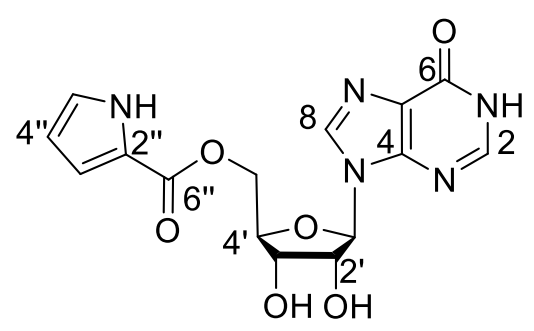

2 
Table S4: Comparison of ${ }^{1} \mathrm{H}(600 \mathrm{MHz})$ NMR shifts for natural and synthetic 2 in DMSO- $\mathrm{d}_{6}$

\begin{tabular}{ccc}
\hline Position & $\begin{array}{c}\text { Natural Sample } \\
{ }^{1} \mathrm{H}\end{array}$ & $\begin{array}{c}\text { Synthetic Sample } \\
{ }^{1} \mathrm{H}\end{array}$ \\
\hline 2 & 8.03 & 7.92 \\
4 & & \\
5 & & \\
6 & & \\
8 & 8.22 & 7.99 \\
$1^{\prime}$ & 5.90 & 5.85 \\
$2^{\prime}$ & 4.57 & 4.62 \\
$3^{\prime}$ & 4.28 & 4.29 \\
$4^{\prime}$ & 4.18 & 4.16 \\
$5^{\prime}$ & 4.49 & 4.48 \\
& 4.36 & 4.35 \\
NH-1" & 11.95 & 11.96 \\
$2^{\prime \prime}$ & & \\
$3^{\prime \prime}$ & 6.80 & 6.82 \\
$4 "$ & 6.18 & 6.18 \\
5" & 7.04 & 7.04 \\
6" & &
\end{tabular}


Figure S12: ${ }^{1} \mathrm{H}$ NMR Spectrum $\left(600 \mathrm{MHz}, \mathrm{DMSO}-\mathrm{d}_{6}\right)$ of 2

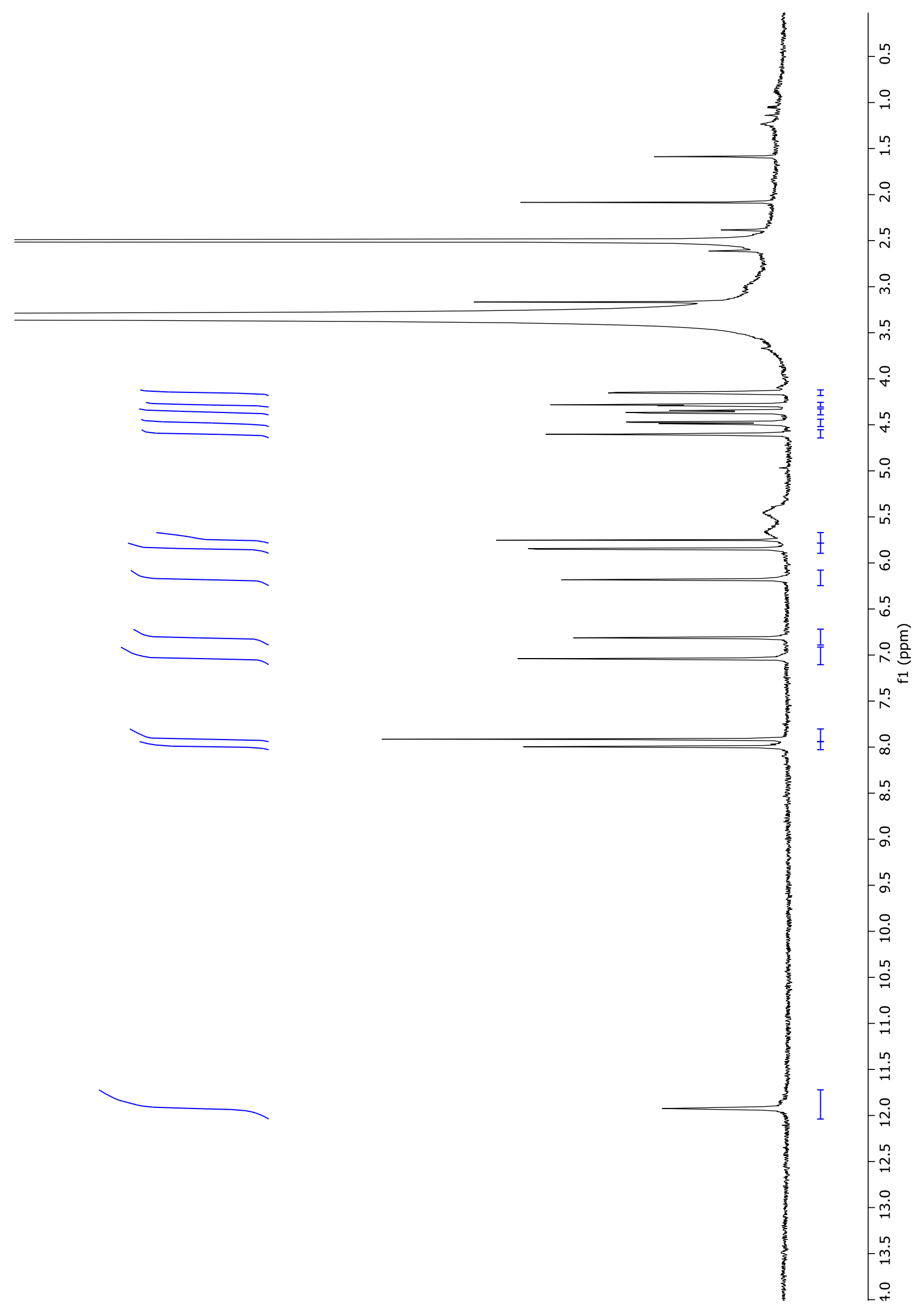


Figure S13: ${ }^{13} \mathrm{C}$ NMR Spectrum (150 MHz, DMSO-d $\left.{ }_{6}\right)$ of 2

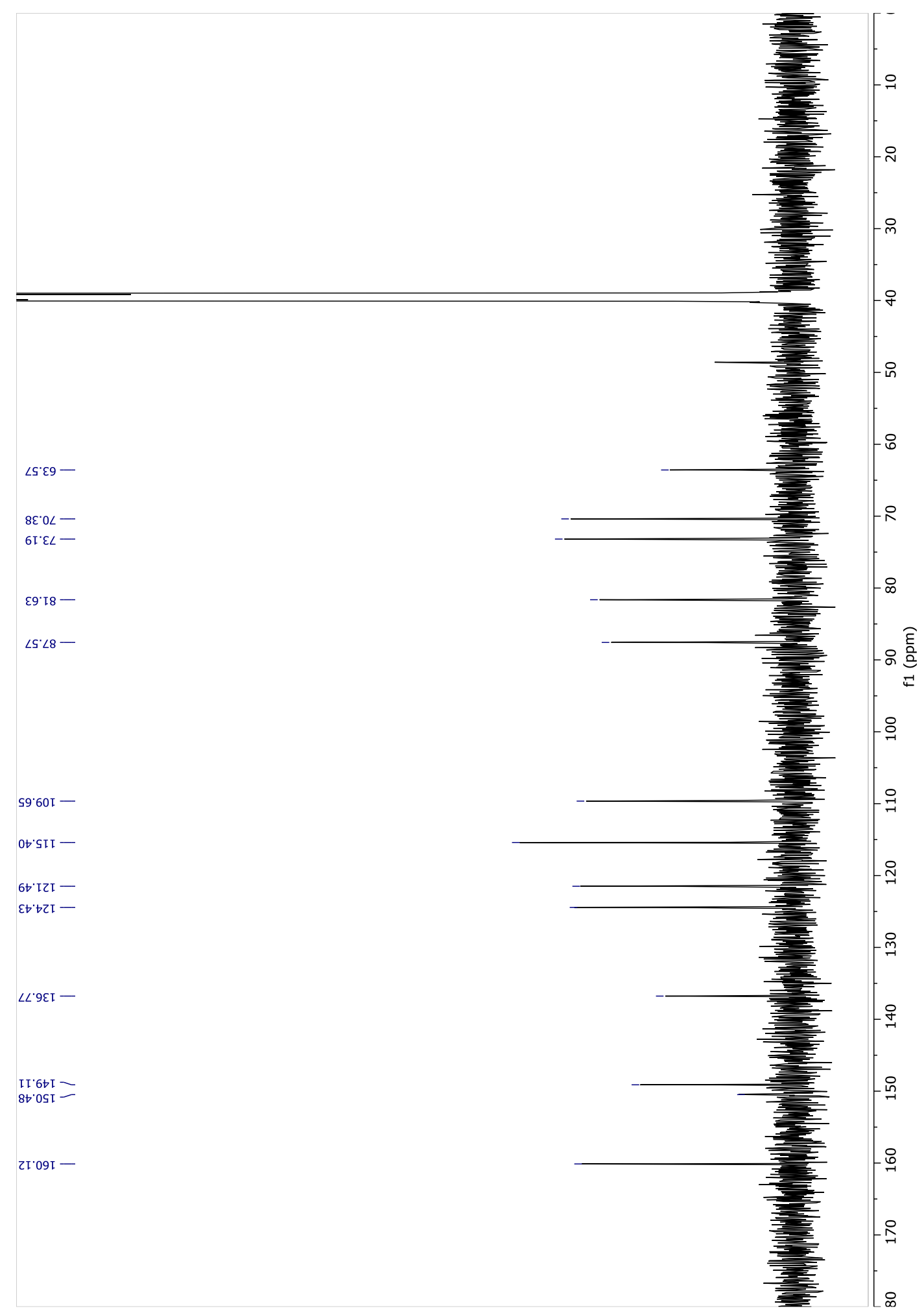


Figure S14: COSY Spectrum (600 MHz, DMSO-d $\left.\mathrm{d}_{6}\right)$ of 2

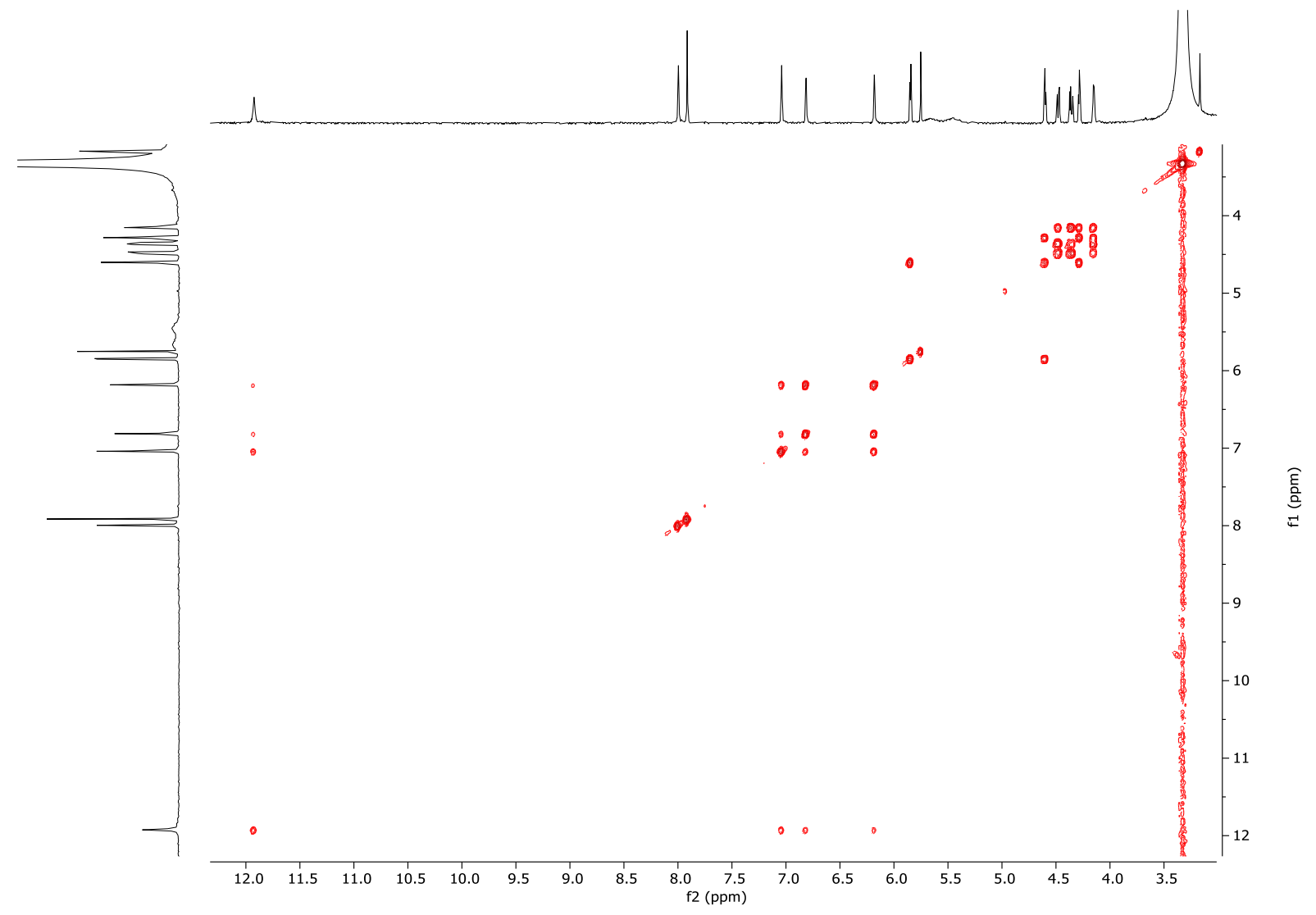

Figure S15: HSQC Spectrum (600 MHz, DMSO-d 6 ) of 2

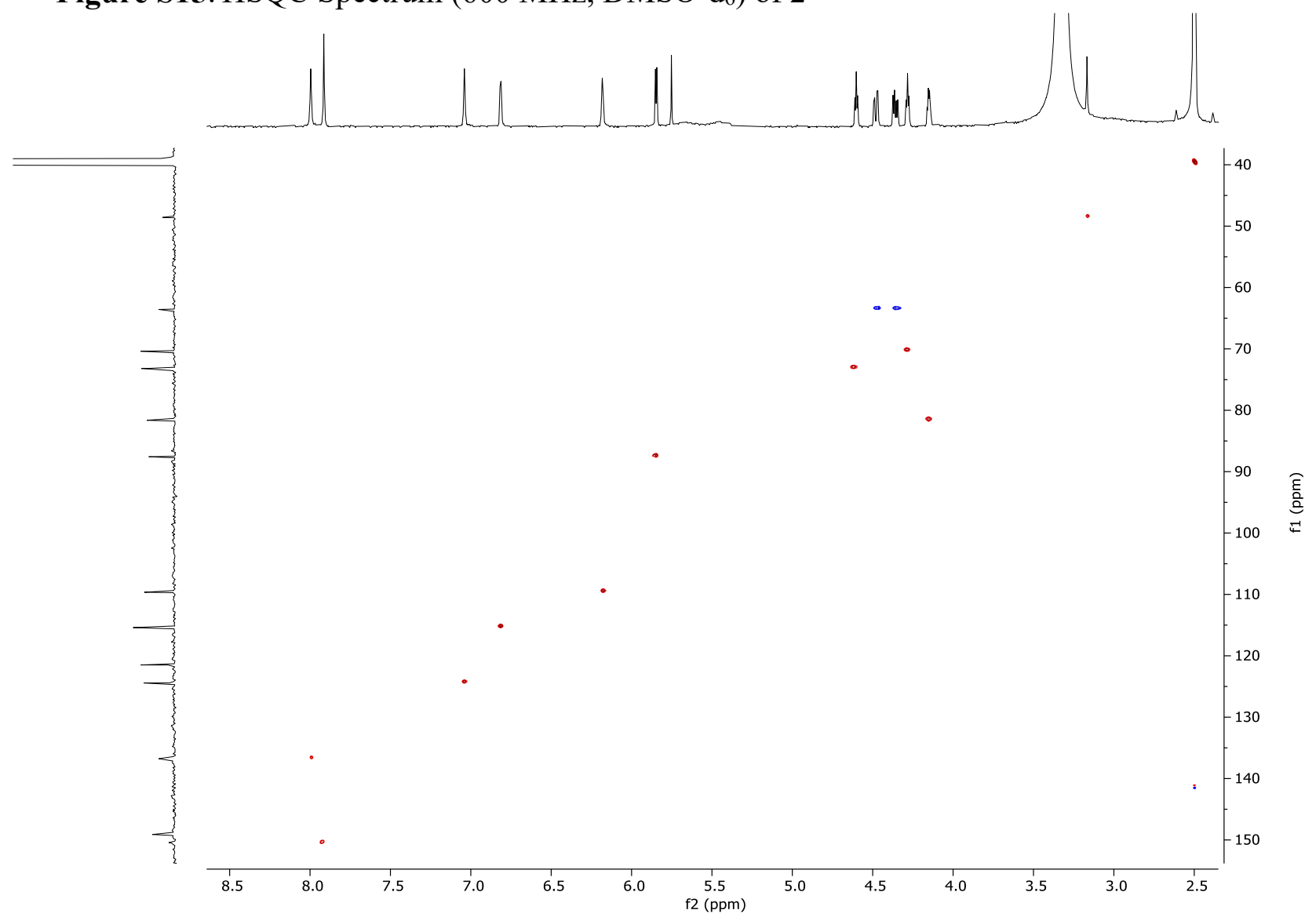


Figure S16: HMBC Spectrum (600 MHz, DMSO-d $\left.\mathrm{d}_{6}\right)$ of 2

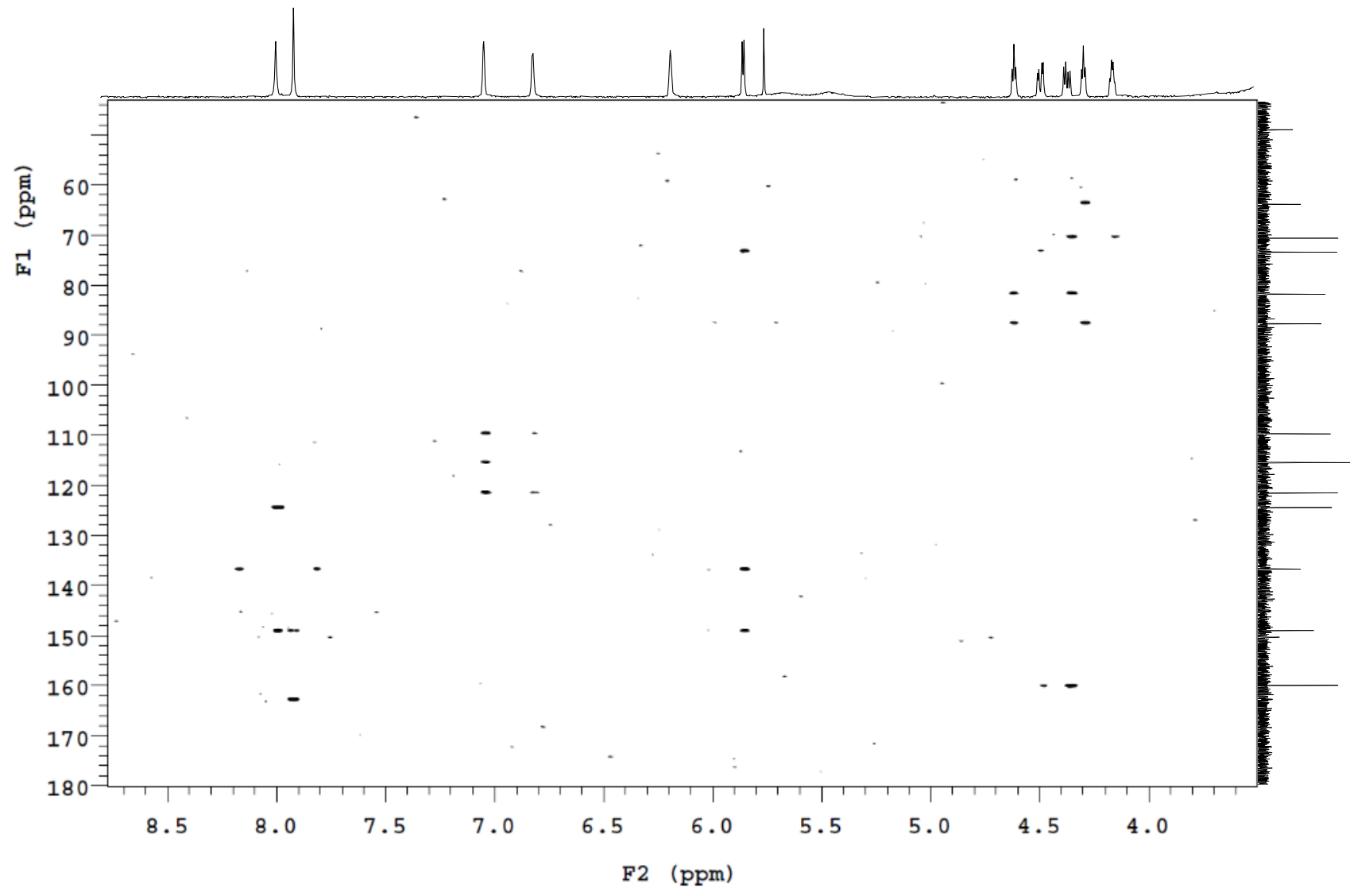

Figure S17: (+)-HRESI-MS spectrum of 2

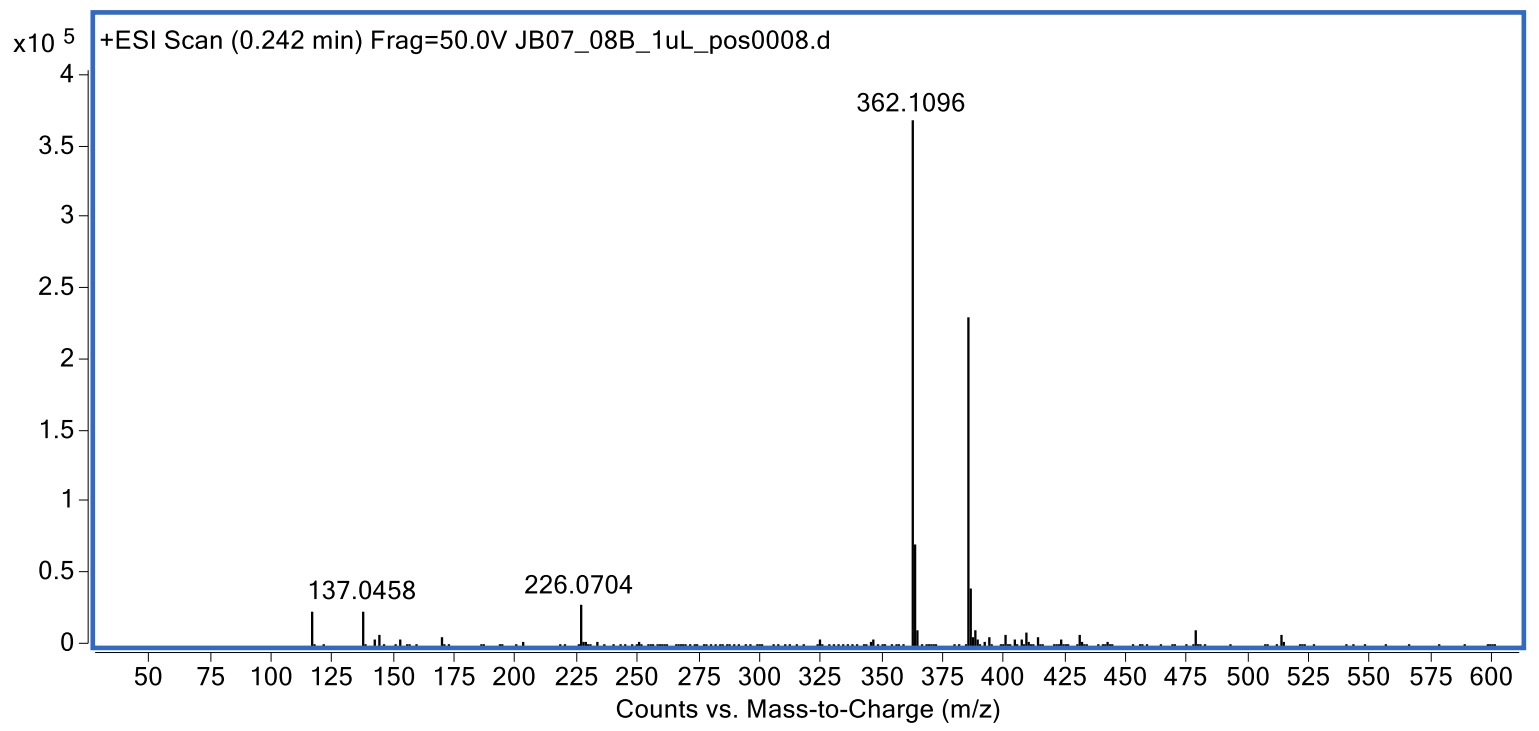


Figure S18: (+)-HRESI-MS/MS spectrum of 2

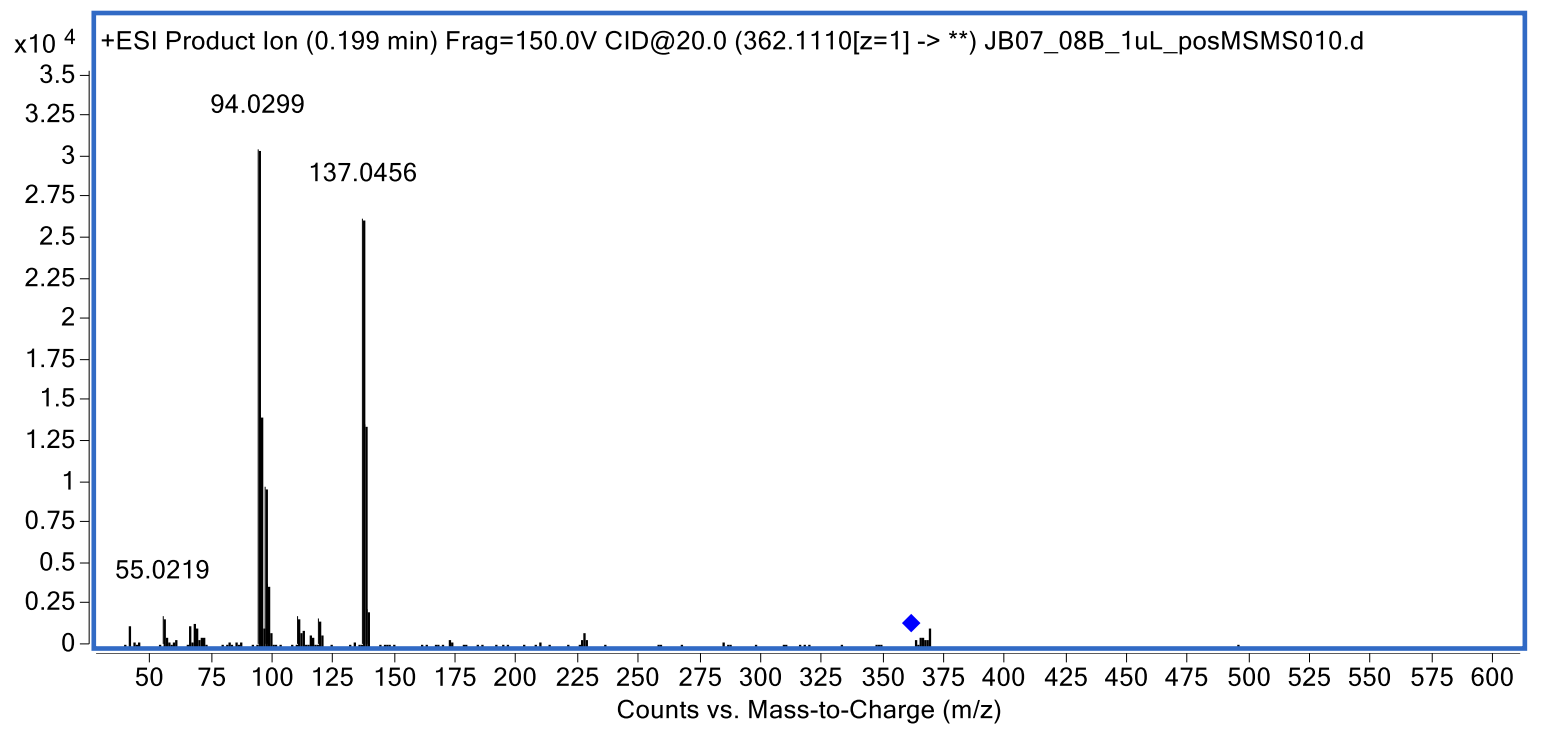

Figure S19: IR spectrum of 2

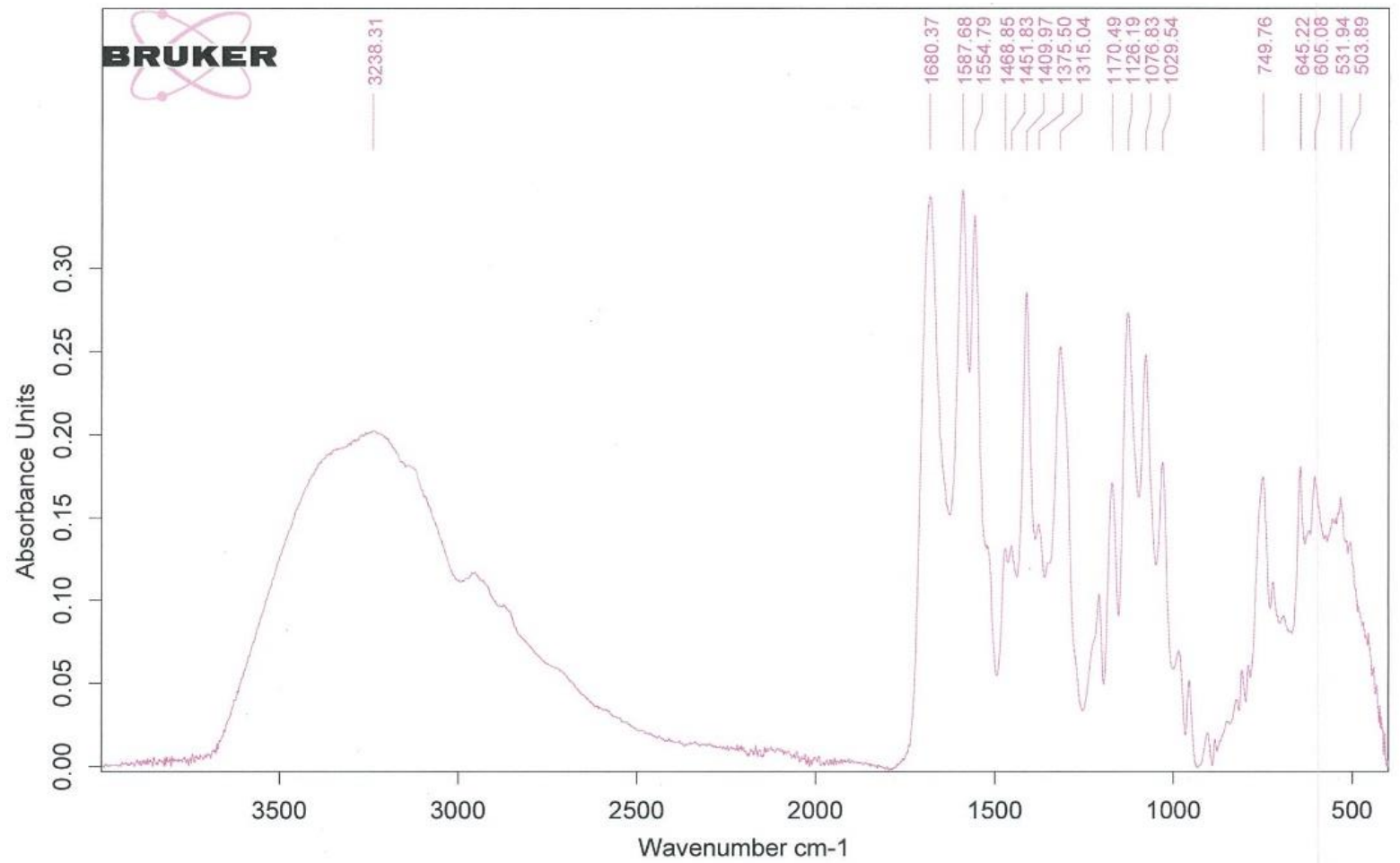


Figure S20: UV/Vis spectrum of 2

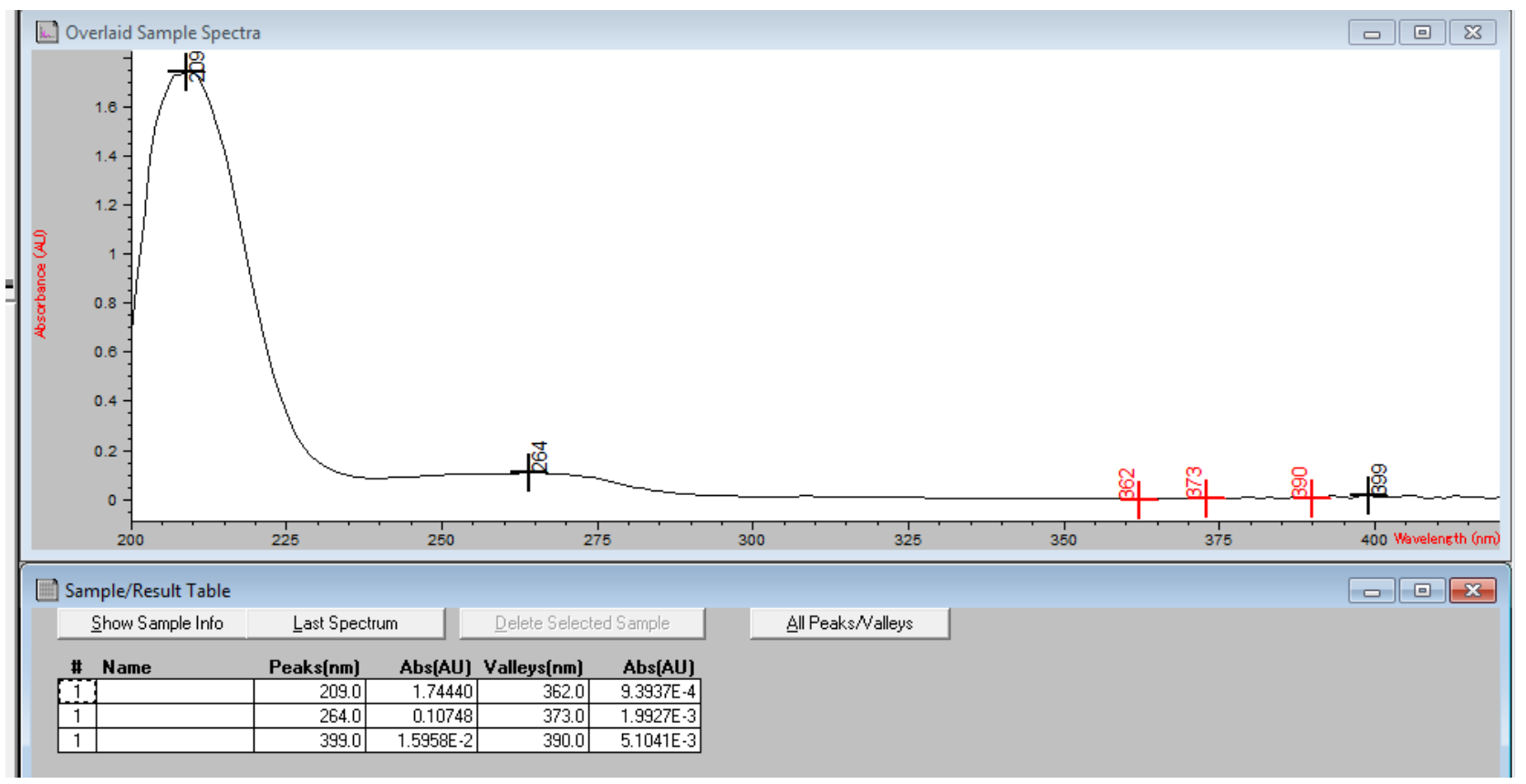


Table S5: ${ }^{13} \mathrm{C}(150 \mathrm{MHz})$ and ${ }^{1} \mathrm{H}(600 \mathrm{MHz})$ NMR data for guanosyl-6'-pyrrole-2-carboxylate (3) in DMSO-d 6

\begin{tabular}{|c|c|c|c|c|}
\hline position & ${ }^{13} \mathrm{C}(\delta)$ & $\begin{array}{c}{ }^{1} \mathrm{H} \\
(\delta, \text { mult., } J \text { in } H z)\end{array}$ & COSY & HMBC \\
\hline 2 & 153.8 & & & \\
\hline $\mathrm{NH}_{2}-2$ & & $6.52(\mathrm{~s})$ & & \\
\hline 4 & 151.3 & & & \\
\hline 5 & 116.7 & & & \\
\hline 6 & 156.8 & & & \\
\hline 8 & 135.6 & $7.82(\mathrm{~s})$ & & 4,5 \\
\hline $1^{\prime}$ & 86.5 & $5.73(\mathrm{~d}, 5.4)$ & $2^{\prime}$ & $4,8,2^{\prime}$ \\
\hline $2^{\prime}$ & 73.3 & $4.46(t, 5.2)$ & $1^{\prime}, 3^{\prime}$ & $1^{\prime}, 3^{\prime}, 4^{\prime}$ \\
\hline $3^{\prime}$ & 70.4 & $4.23(\mathrm{t}, 4.8)$ & $2^{\prime}, 4^{\prime}$ & $1^{\prime}, 5^{\prime}$ \\
\hline $4^{\prime}$ & 81.7 & $4.12(\mathrm{dt}, 4.1,5.6)$ & $3^{\prime}, 5^{\prime}$ & $1^{\prime}, 5^{\prime}$ \\
\hline \multirow[t]{2}{*}{$5^{\prime}$} & 63.5 & $4.48(\mathrm{dd}, 12.0,3.8)$ & $4^{\prime}$ & 3', 6" \\
\hline & & $4.34(\mathrm{dd}, 12.0,5.7)$ & $4^{\prime}$ & $3^{\prime}, 4^{\prime}, 6^{\prime \prime}$ \\
\hline NH-1" & & $11.9(\mathrm{~s})$ & 3", 4", 5" & \\
\hline $2^{\prime \prime}$ & 121.4 & & & \\
\hline $3 "$ & 115.4 & $6.81(\mathrm{~m})$ & NH-1", 4", 5" & \\
\hline $4 "$ & 109.7 & $6.19(\mathrm{~m})$ & NH-1", 3", 5" & \\
\hline $5^{\prime \prime}$ & 124.5 & $7.04(\mathrm{~m})$ & NH-1", 3", 4" & \\
\hline $6^{\prime \prime}$ & 160.1 & & & \\
\hline
\end{tabular}

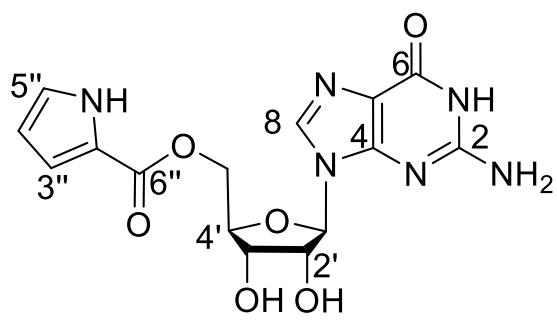


Figure S21: ${ }^{1} \mathrm{H}$ NMR Spectrum (600 MHz, DMSO-d 6 ) of 3

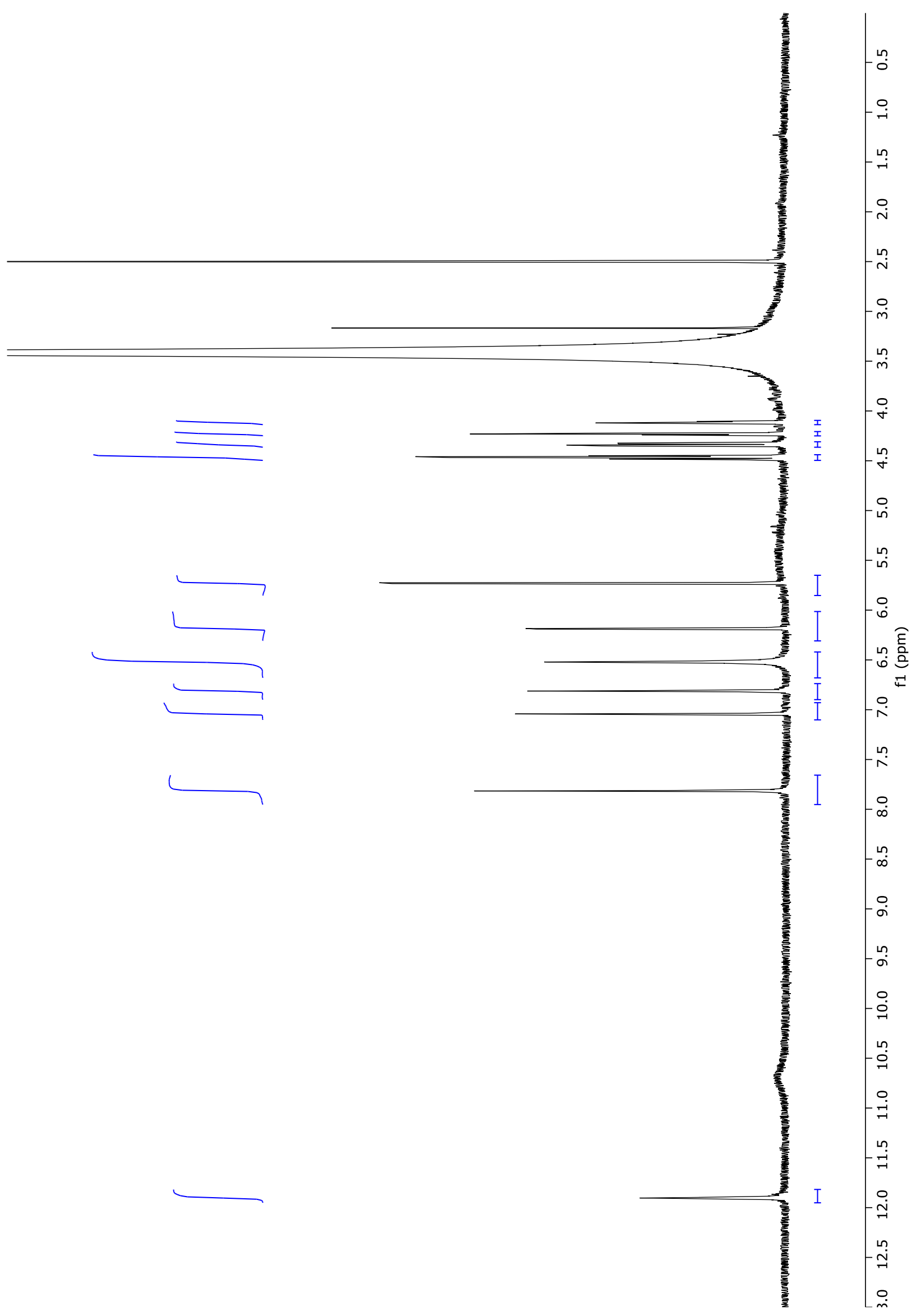


Figure S22: ${ }^{13} \mathrm{C}$ NMR Spectrum (150 MHz, DMSO-d $\left.{ }_{6}\right)$ of 3

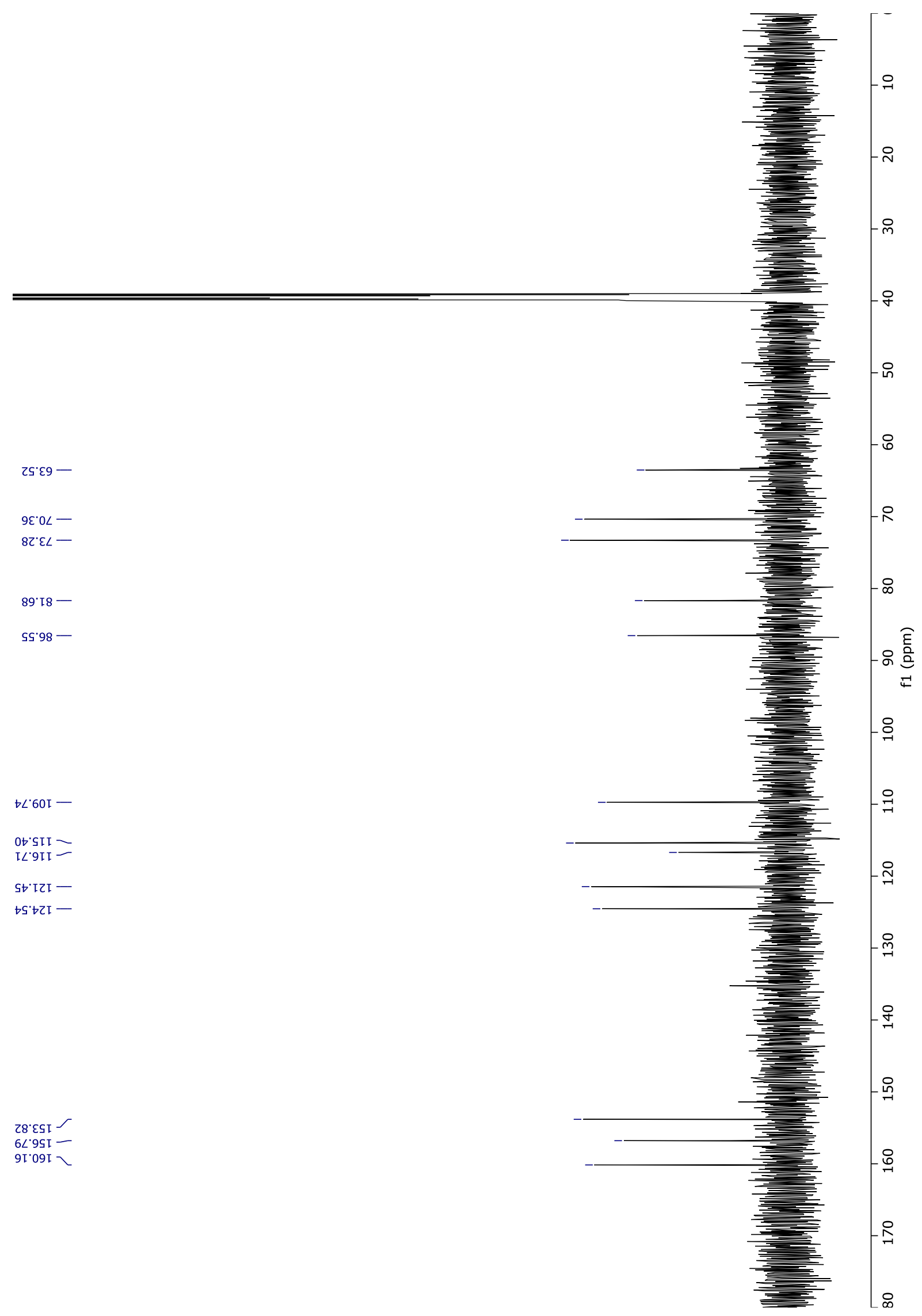


Figure S23: COSY Spectrum (600 MHz, DMSO-d $\left.\mathrm{d}_{6}\right)$ of 3

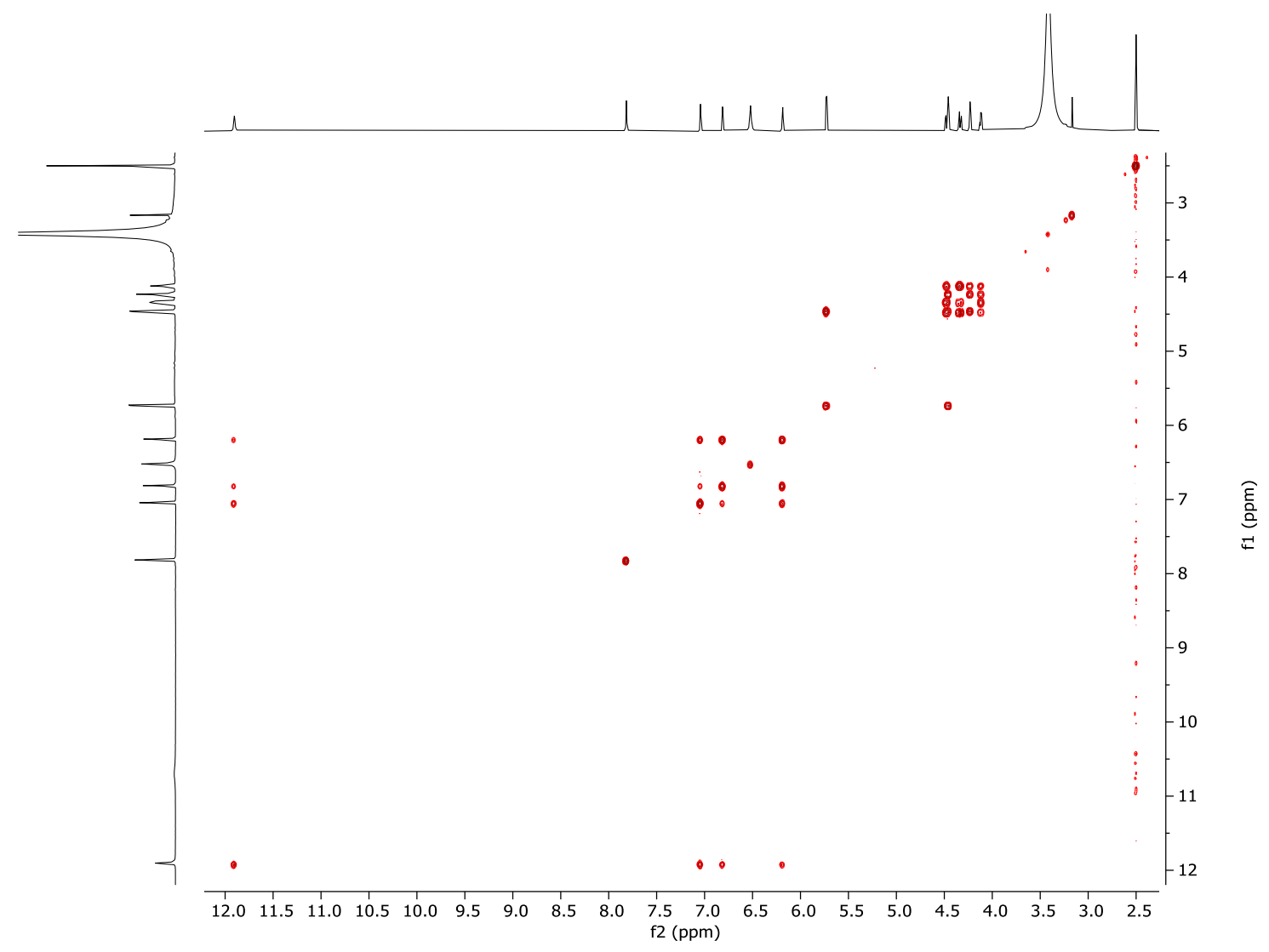

Figure S24: HSQC Spectrum (600 MHz, DMSO-d 6 ) of 3

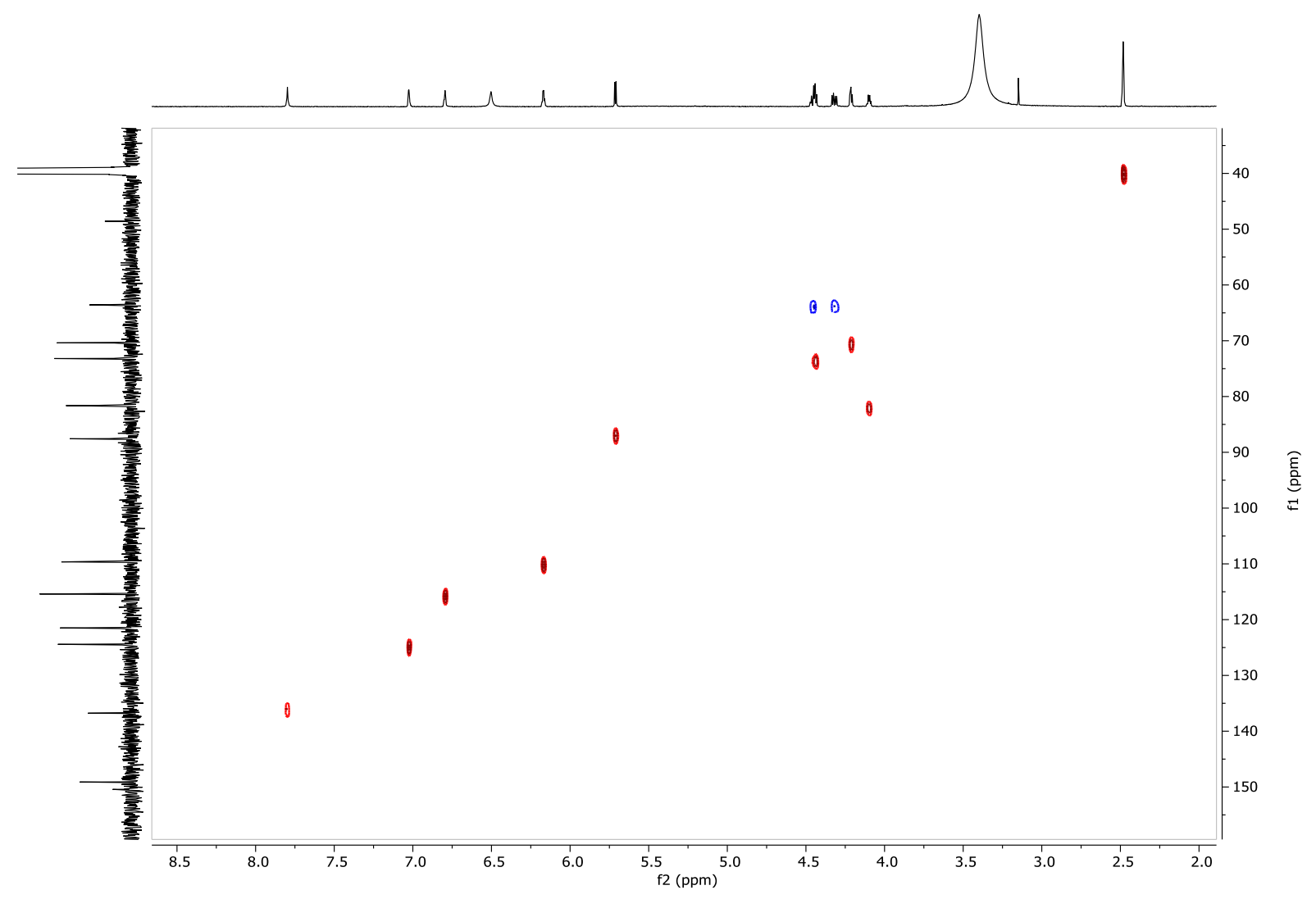


Figure S25: HMBC Spectrum (600 MHz, DMSO-d $\left.{ }_{6}\right)$ of 3

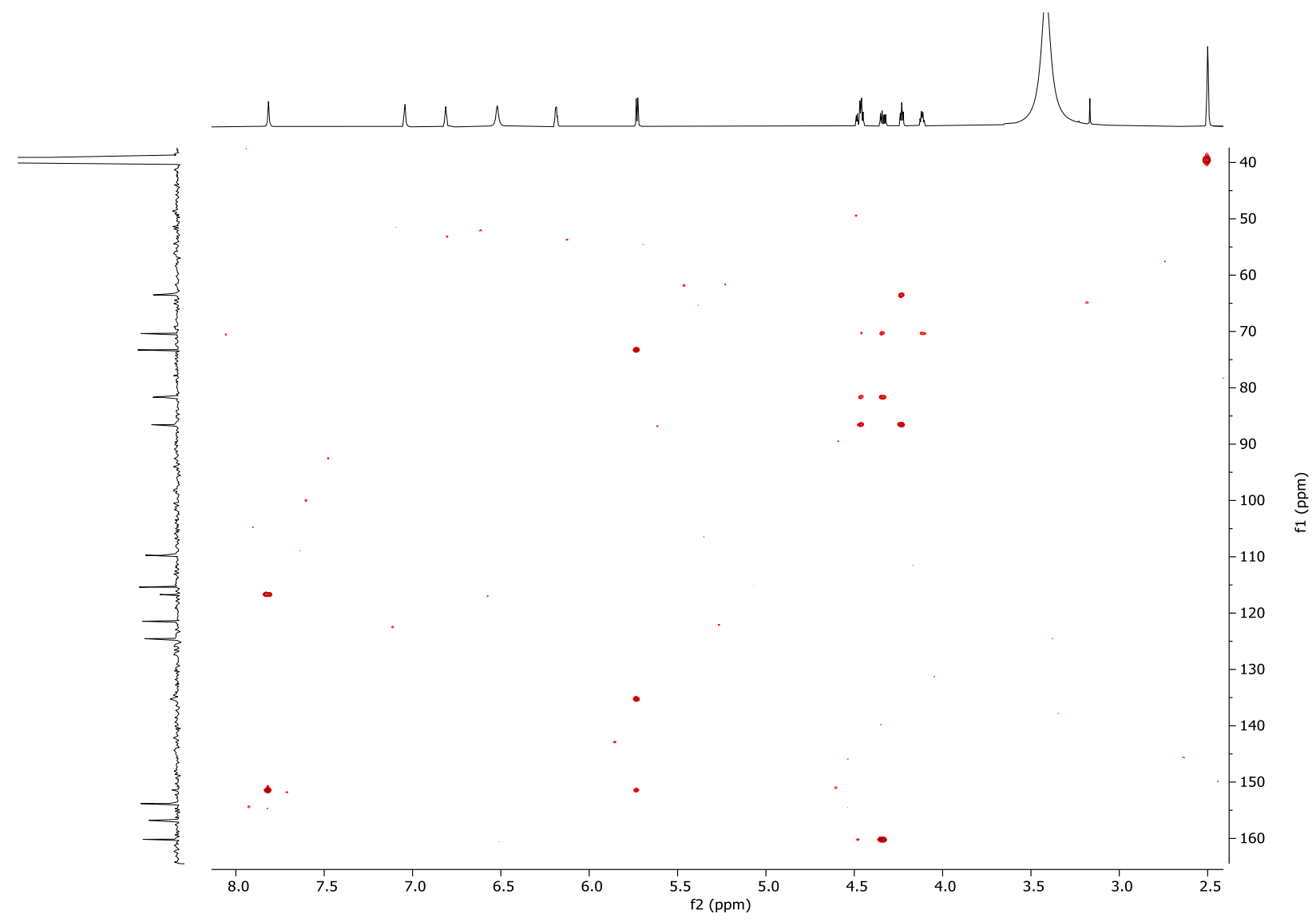

Figure S26: (+)-HRESIMS spectrum of 3

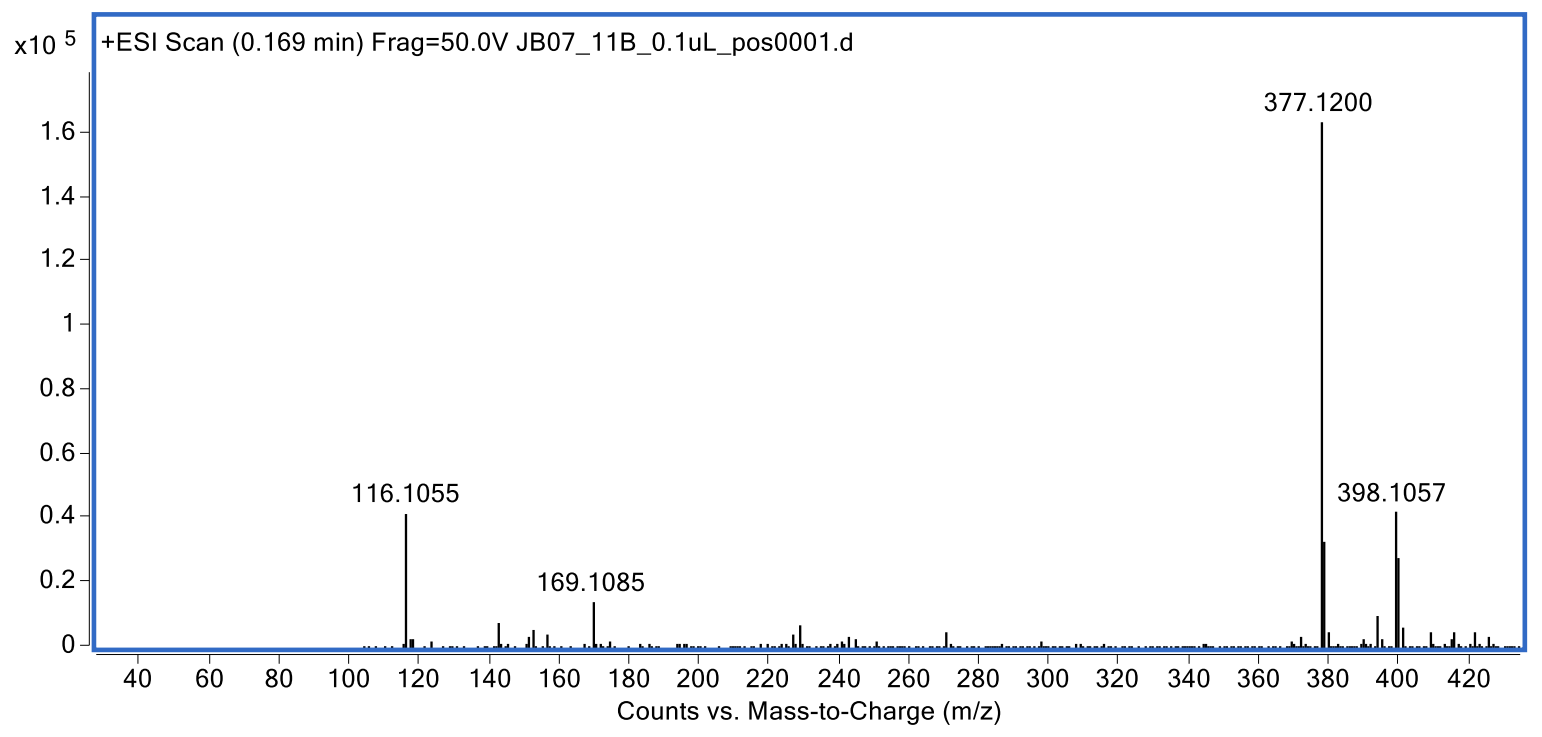


Figure S27: (+)-HRESI-MS/MS spectrum of 3

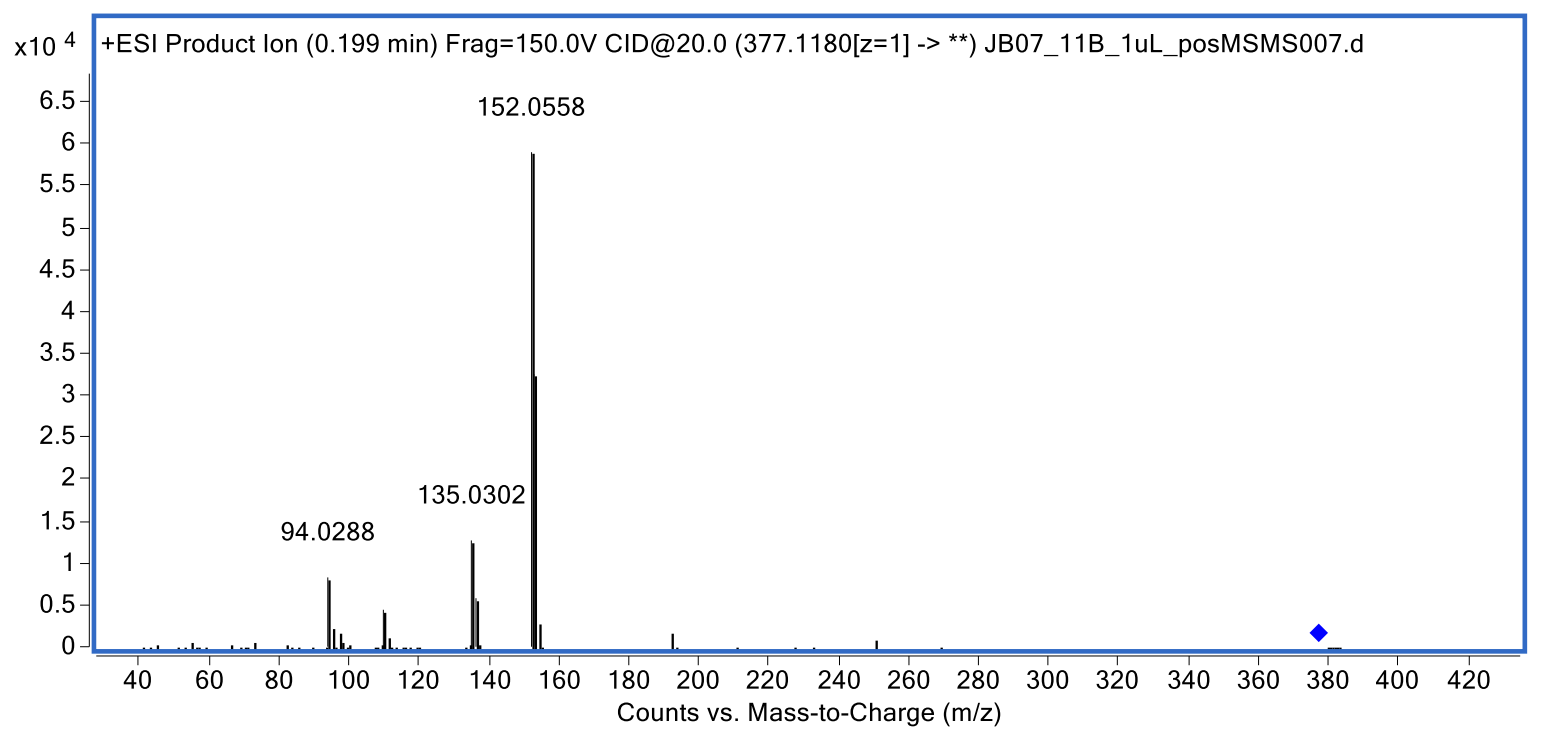

Figure S28: IR spectrum of $\mathbf{3}$

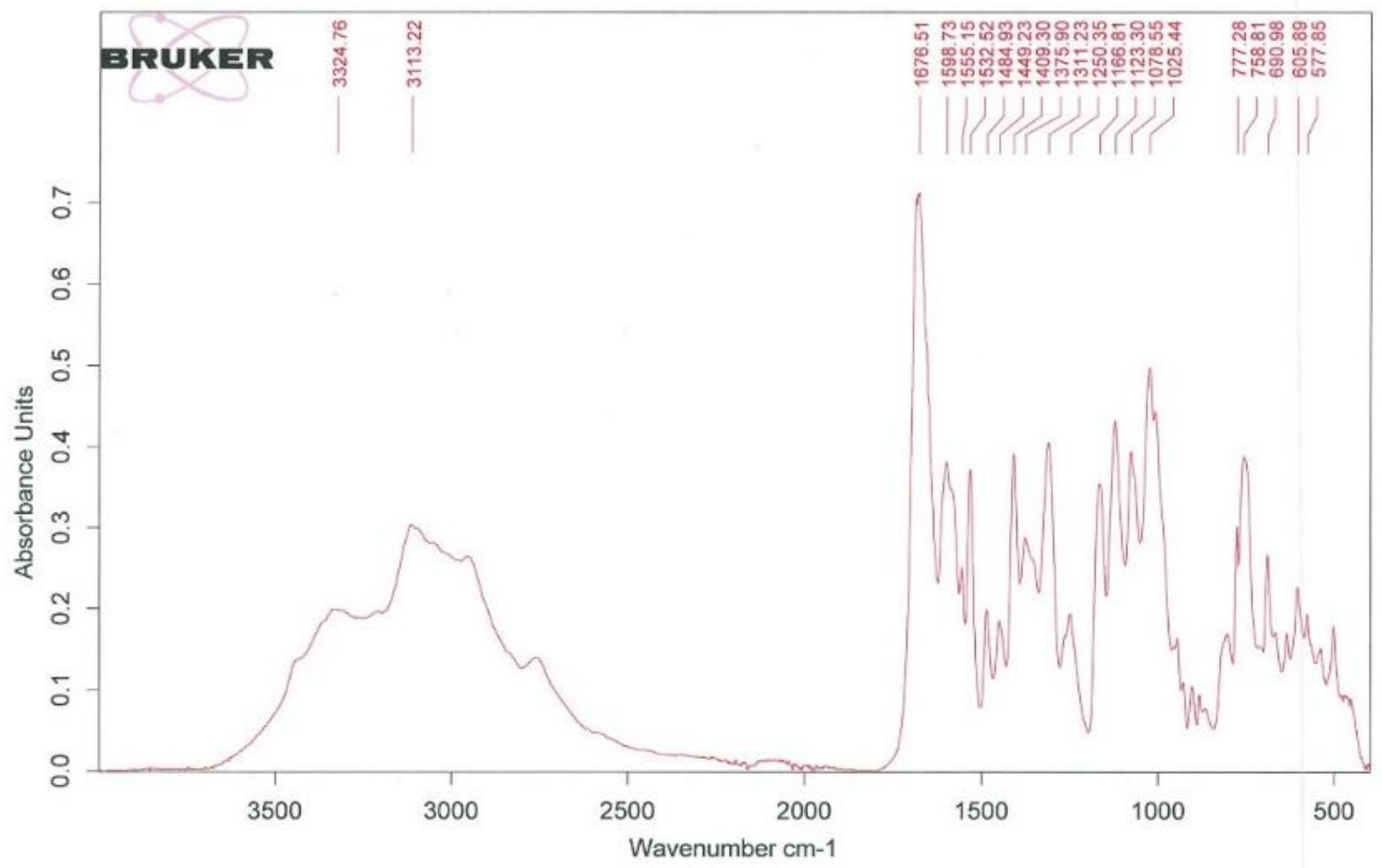


Figure S29: UV/Vis spectrum of 3

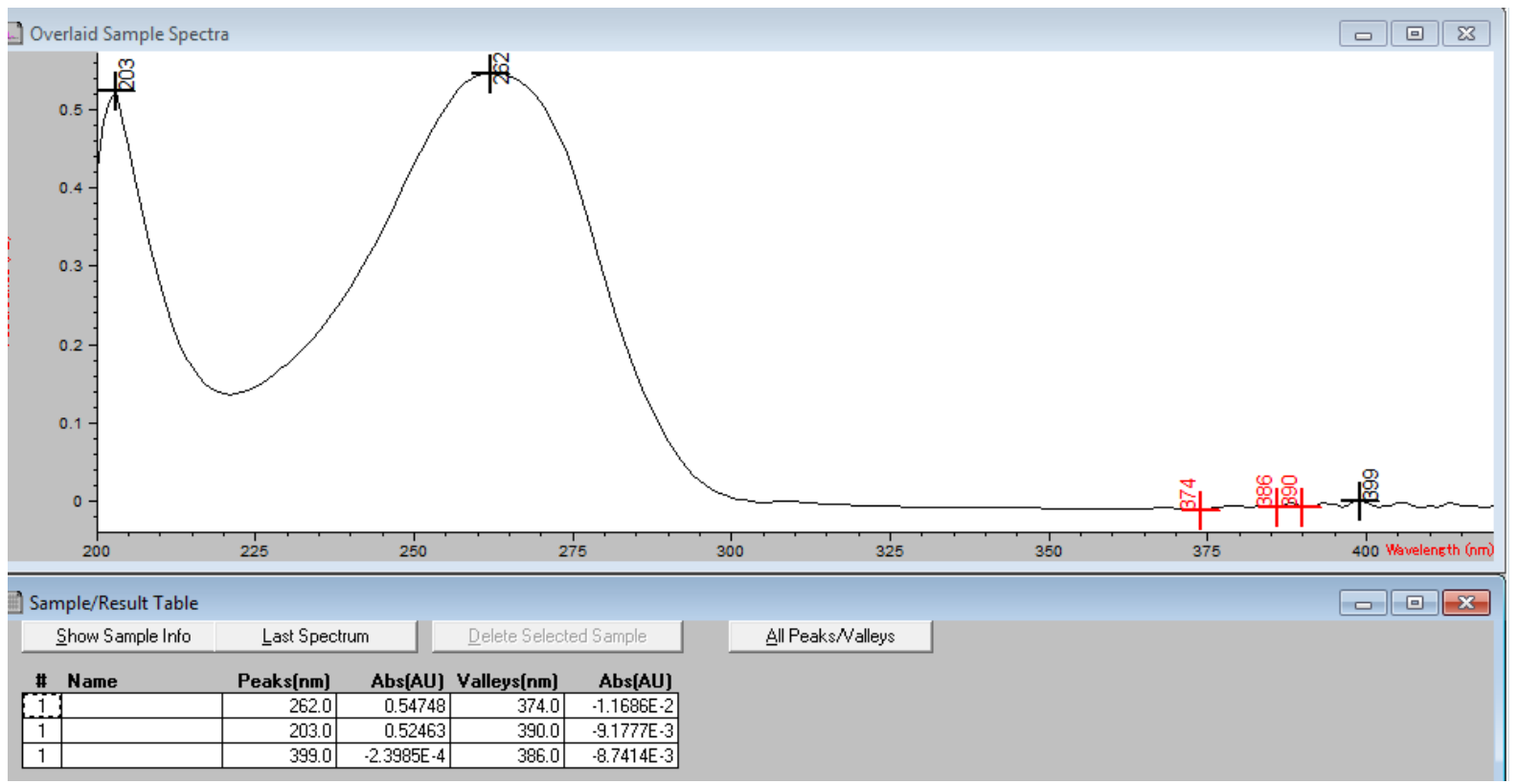


Table S6: ${ }^{13} \mathrm{C}(150 \mathrm{MHz})$ and ${ }^{1} \mathrm{H}(600 \mathrm{MHz}) \mathrm{NMR}$ data for adenosyl-6'-pyrrole-3-carboxylate (4) in DMSO-d 6

\begin{tabular}{|c|c|c|c|c|}
\hline position & ${ }^{13} \mathrm{C}(\delta)$ & $\begin{array}{c}{ }^{1} \mathrm{H} \\
(\delta, \text { mult., } J \text { in } H z)\end{array}$ & COSY & HMBC \\
\hline 2 & 152.9 & $8.16(\mathrm{~s})$ & & 4,6 \\
\hline 4 & 149.6 & & & \\
\hline 5 & 119.3 & & & \\
\hline 6 & 156.2 & & & \\
\hline $\mathrm{NH}_{2}-6$ & & $7.27(\mathrm{~s})$ & & 5 \\
\hline 8 & 139.6 & $8.24(\mathrm{~s})$ & & 4,5 \\
\hline $1^{\prime}$ & 87.7 & $5.92(\mathrm{~d}, 5.1)$ & $2^{\prime}$ & $4,8,2^{\prime}, 3^{\prime}$ \\
\hline $2^{\prime}$ & 73.3 & $4.67(\mathrm{t}, 5.2)$ & $1^{\prime}, 3^{\prime}$ & $1^{\prime}, 4^{\prime}$ \\
\hline $3^{\prime}$ & 70.5 & $4.31(\mathrm{~m})$ & $2^{\prime}, 4^{\prime}$ & $1^{\prime}, 4^{\prime}$ \\
\hline $4^{\prime}$ & 82.1 & $4.17(\mathrm{~m})$ & $3^{\prime}, 5^{\prime}$ & $3^{\prime}, 5^{\prime}$ \\
\hline \multirow[t]{2}{*}{$5^{\prime}$} & 63.1 & $4.43(\mathrm{dd}, 12.1,3.6)$ & $4^{\prime}$ & $3^{\prime}, 4^{\prime}, 6^{\prime \prime}$ \\
\hline & & $4.32(\mathrm{~m})$ & $4^{\prime}$ & $3^{\prime}, 4^{\prime}, 6^{\prime \prime}$ \\
\hline NH-1" & & $11.45(\mathrm{~s})$ & 2", 4", 5" & \\
\hline $2^{\prime \prime}$ & 124.2 & $7.45(\mathrm{~m})$ & NH-1", 4", 5" & 3", 4", 5" \\
\hline $3 "$ & 114.5 & & & \\
\hline $4 "$ & 109 & $6.44(\mathrm{~m})$ & NH-1", 2", 5" & 2", 3", 5" \\
\hline $5^{\prime \prime}$ & 119.7 & $6.82(\mathrm{~m})$ & NH-1", 2", 4" & 2", 3", 4" \\
\hline 6" & 164.2 & & & \\
\hline & & $\underbrace{\mathrm{O}^{\prime}}_{\mathrm{OH} \mathrm{OH}}$ & & \\
\hline & & 4 & & \\
\hline
\end{tabular}


Figure S30: ${ }^{1} \mathrm{H}$ NMR Spectrum $\left(600 \mathrm{MHz}, \mathrm{DMSO}-\mathrm{d}_{6}\right)$ of 4

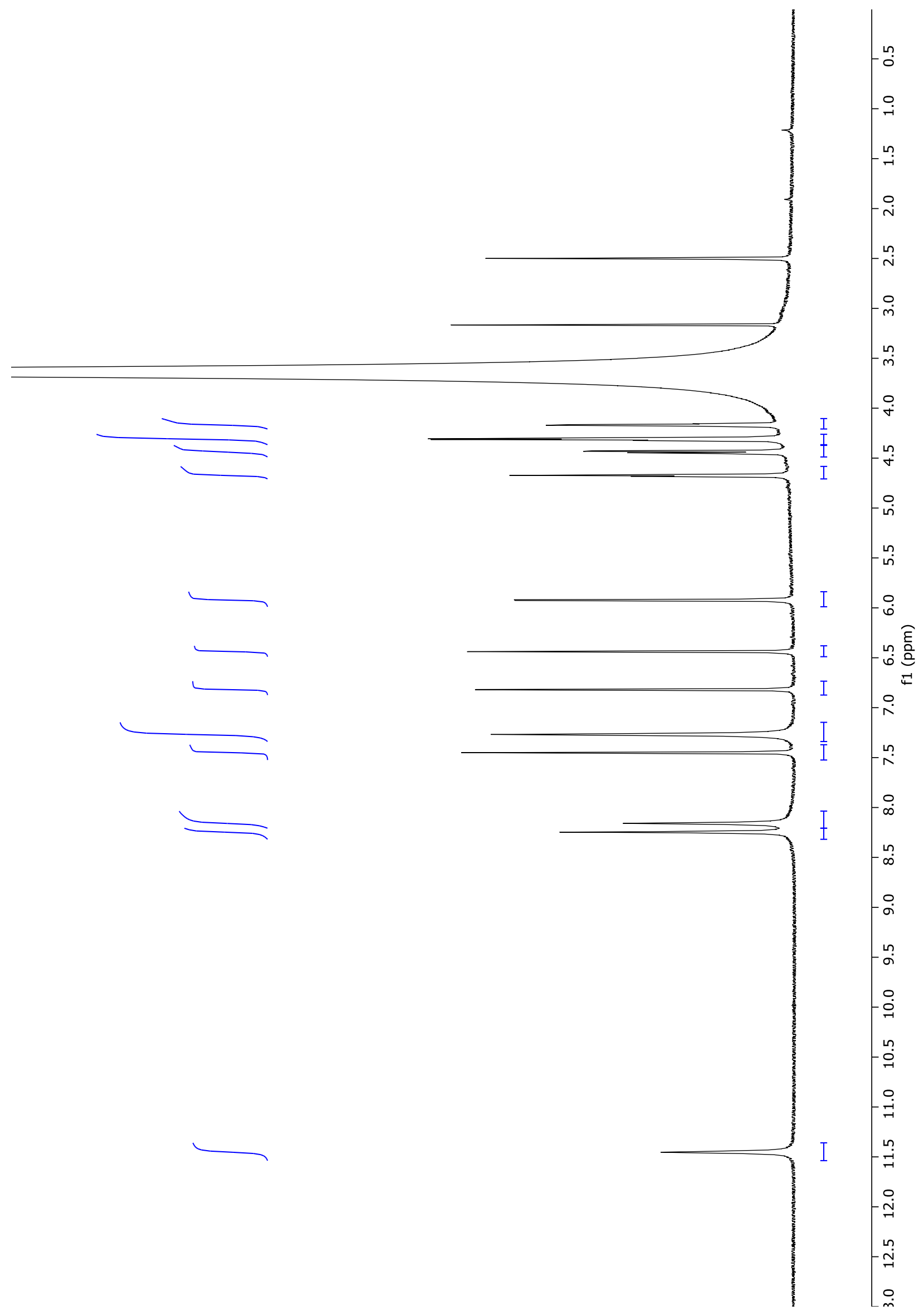


Figure S31: ${ }^{13} \mathrm{C}$ NMR Spectrum (150 MHz, DMSO-d $\left.\mathrm{d}_{6}\right)$ of 4

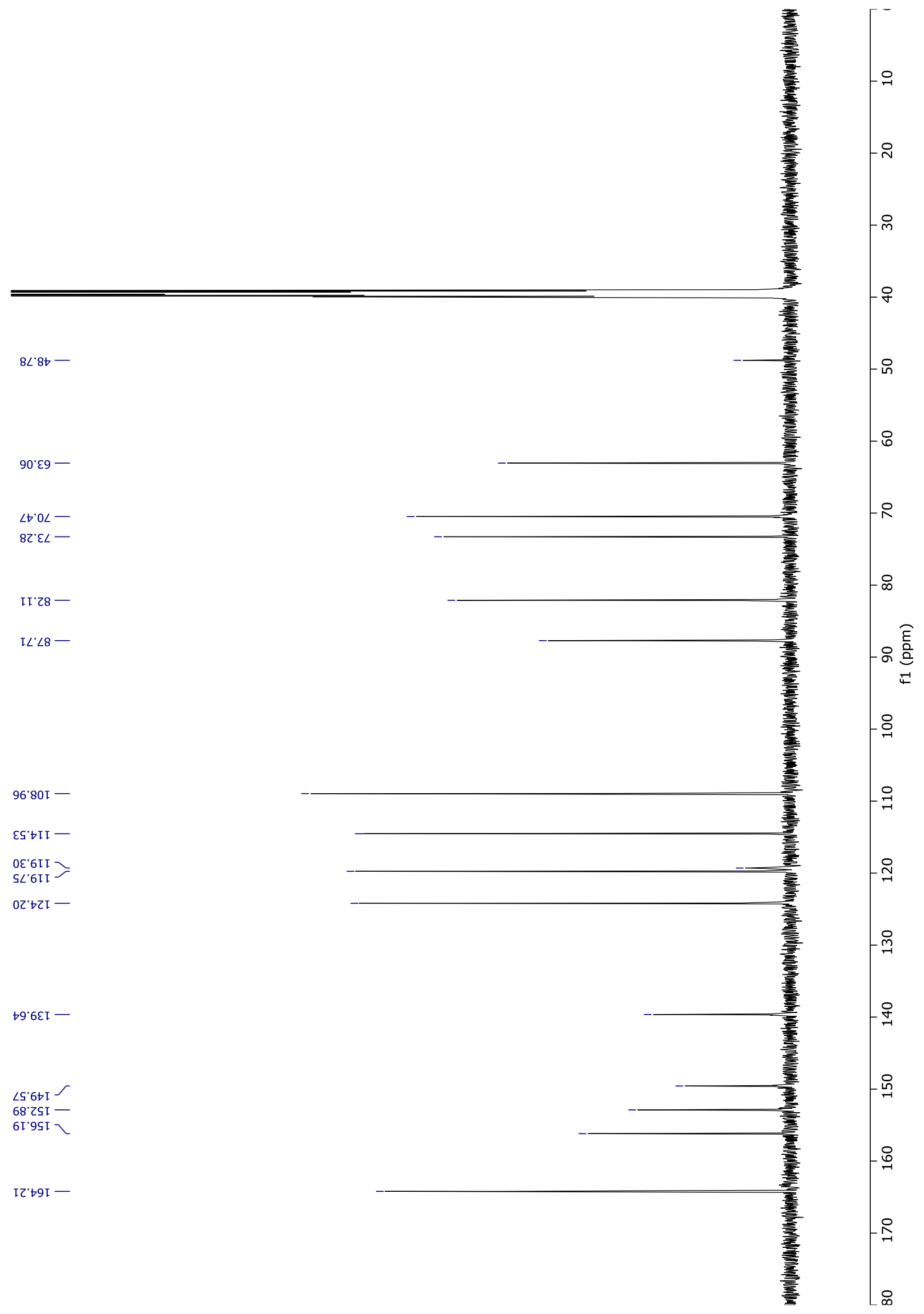


Figure S32: COSY Spectrum (600 MHz, DMSO-d $\left.{ }_{6}\right)$ of 4

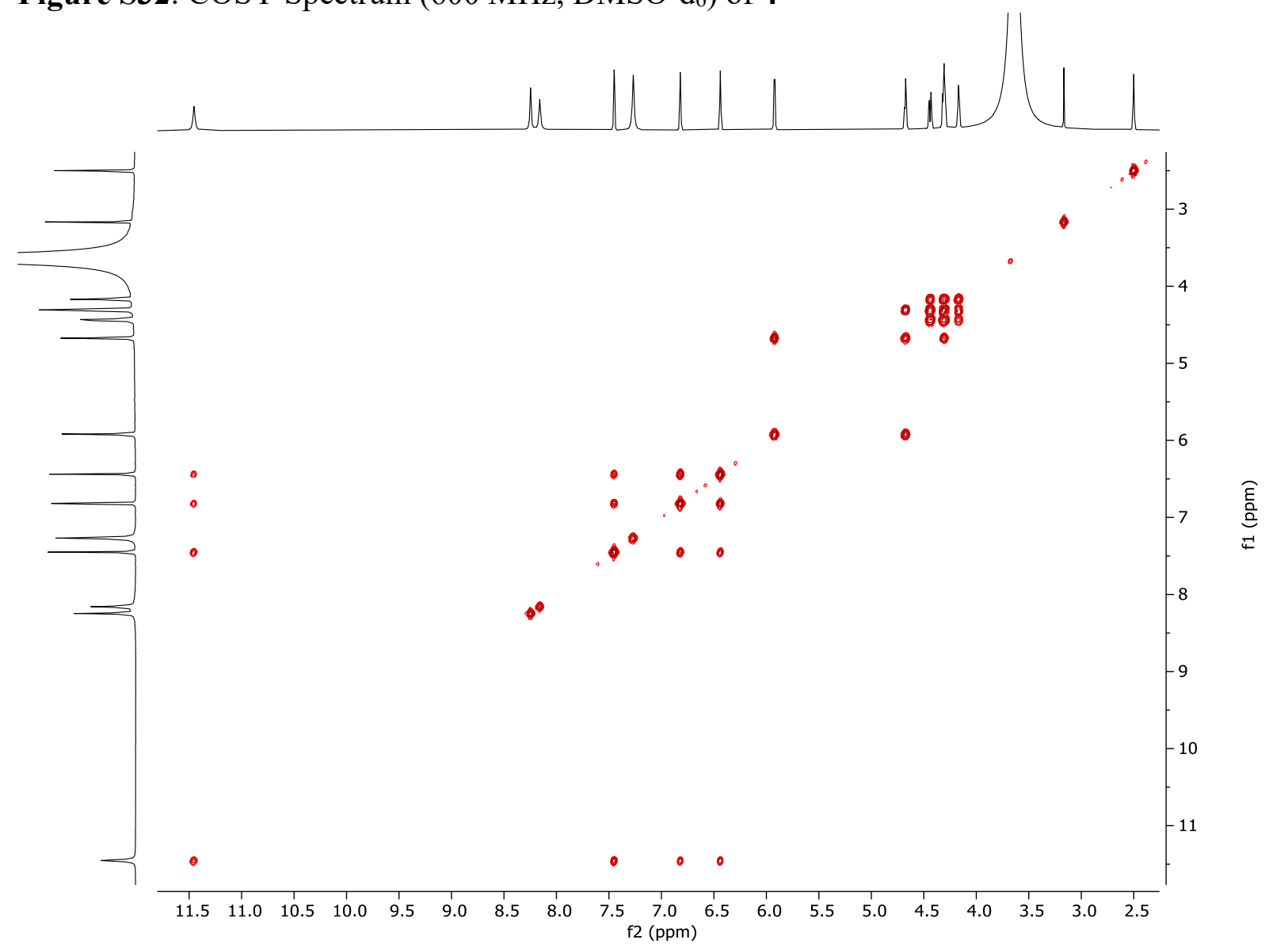

Figure S33: HSQC Spectrum (600 MHz, DMSO-d 6 ) of 4

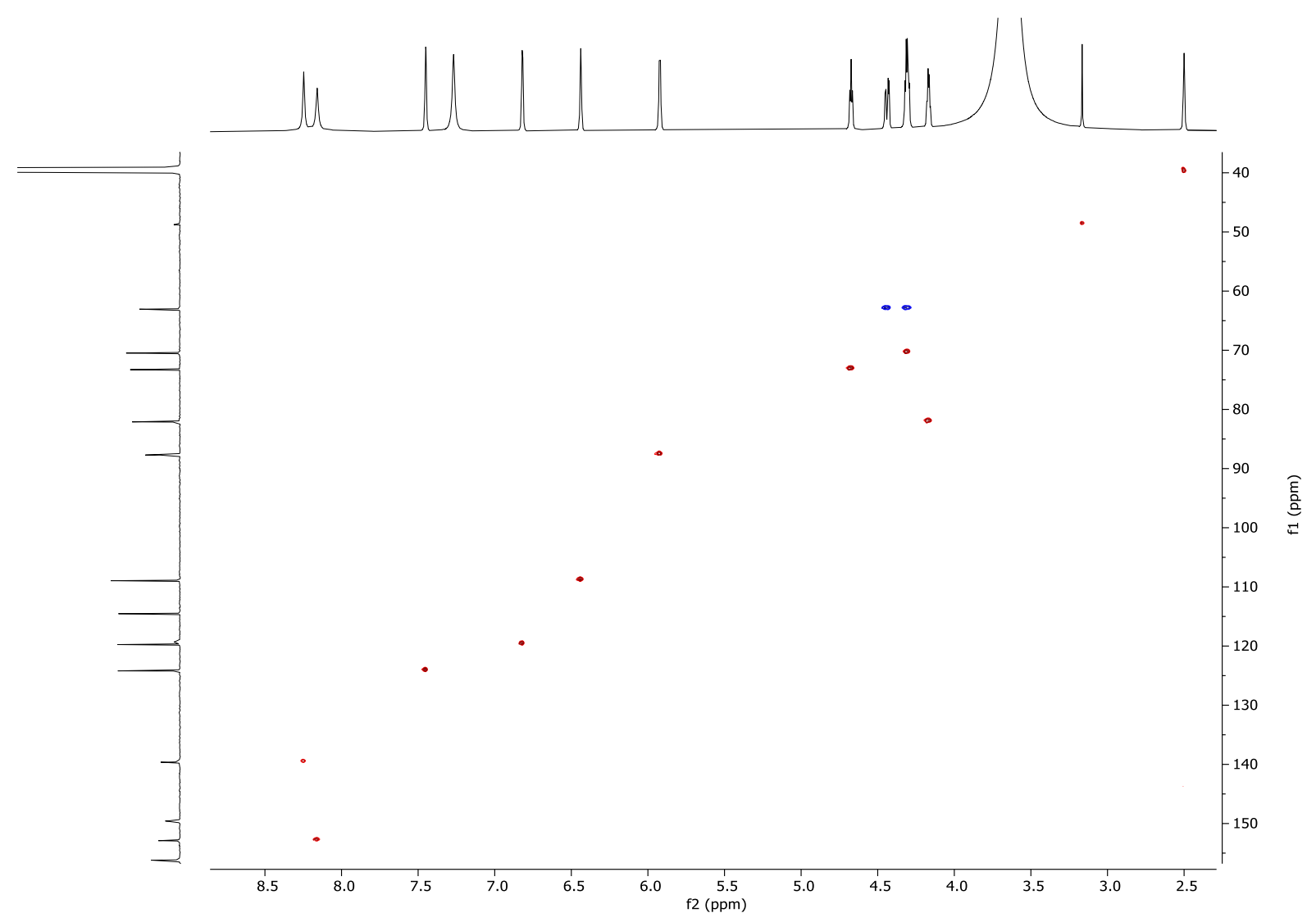


Figure S34: HMBC Spectrum (600 MHz, DMSO-d $\left.\mathrm{d}_{6}\right)$ of 4

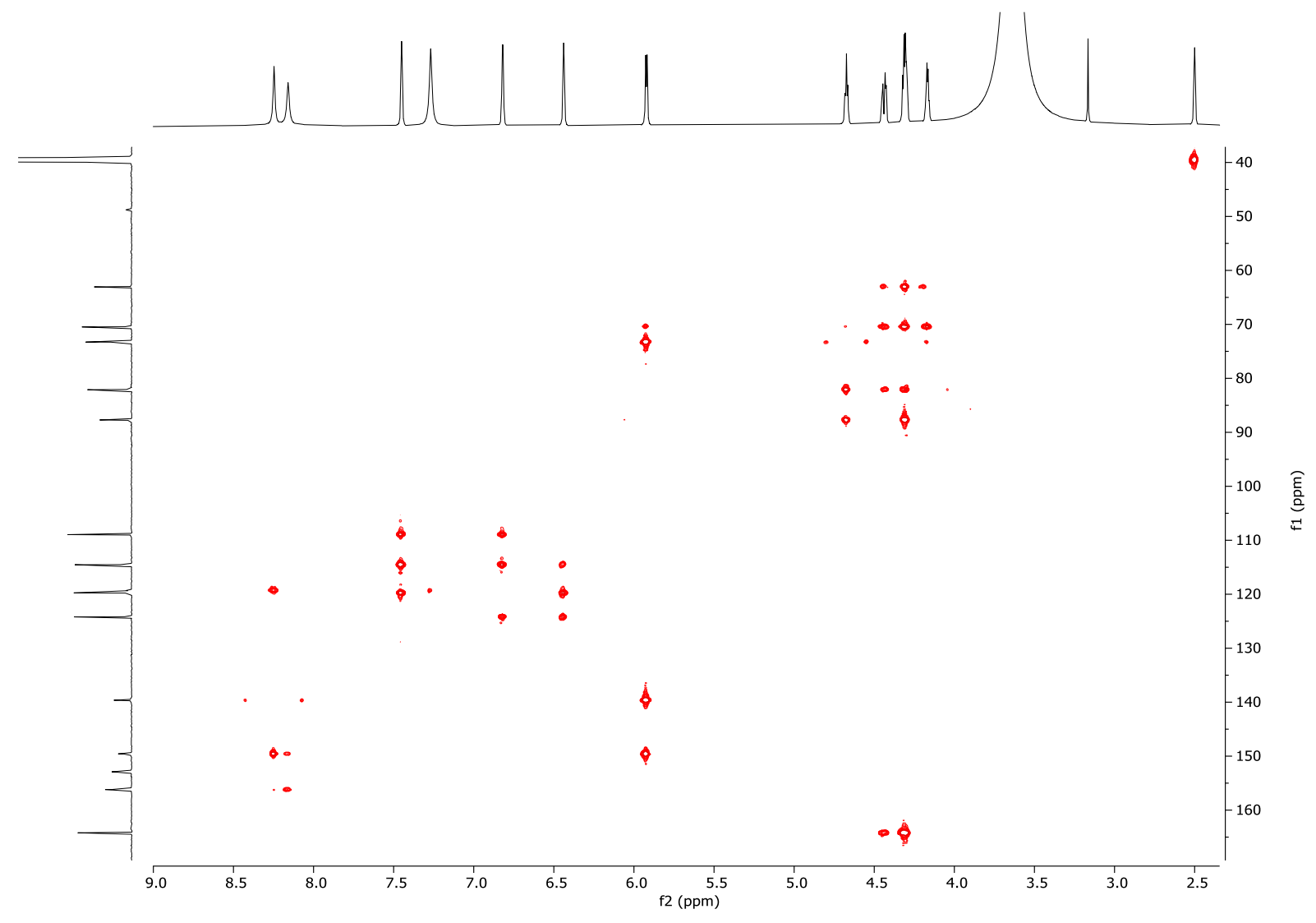

Figure S35: (+)-HRESIMS spectrum of 4

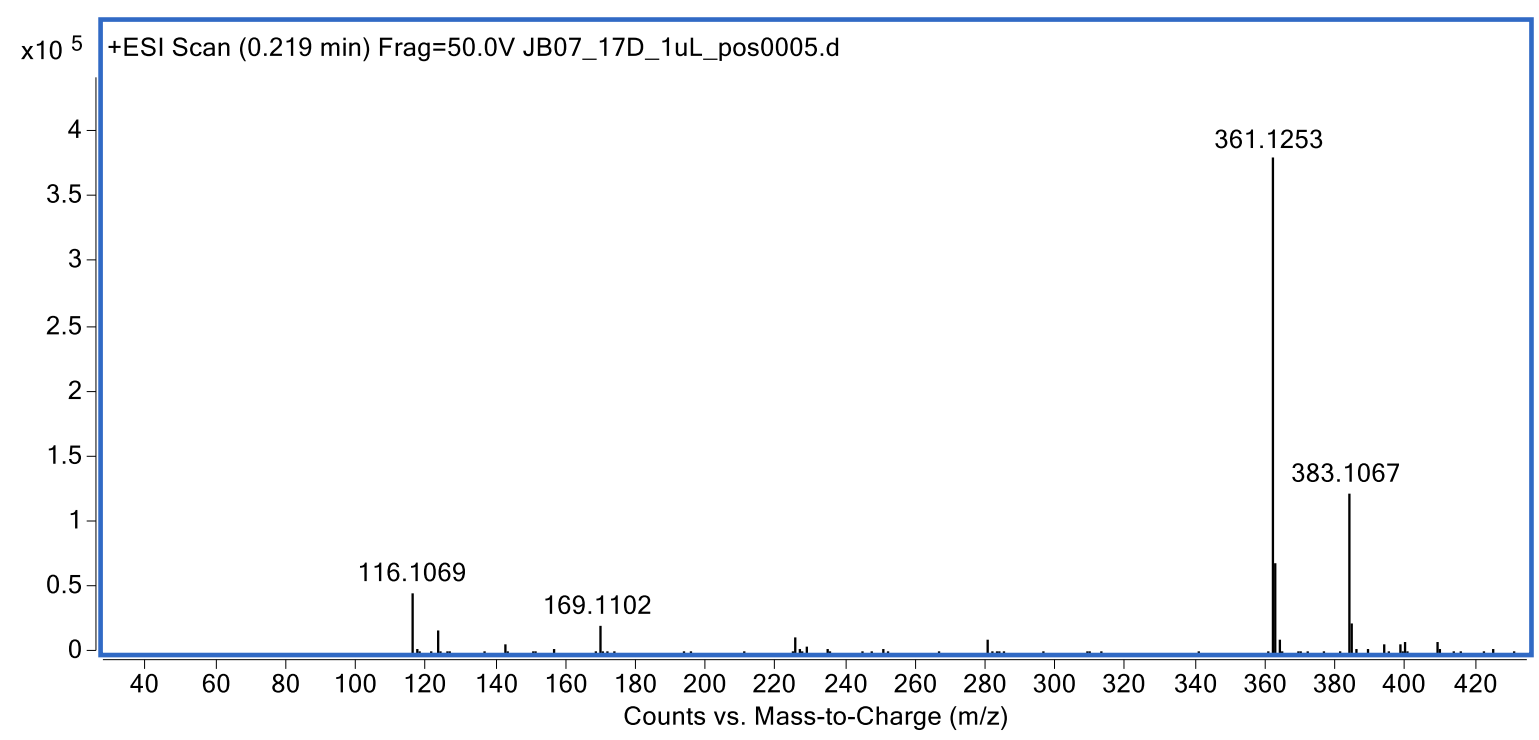


Figure S36: (+)-HRESI-MS/MS spectrum of 4

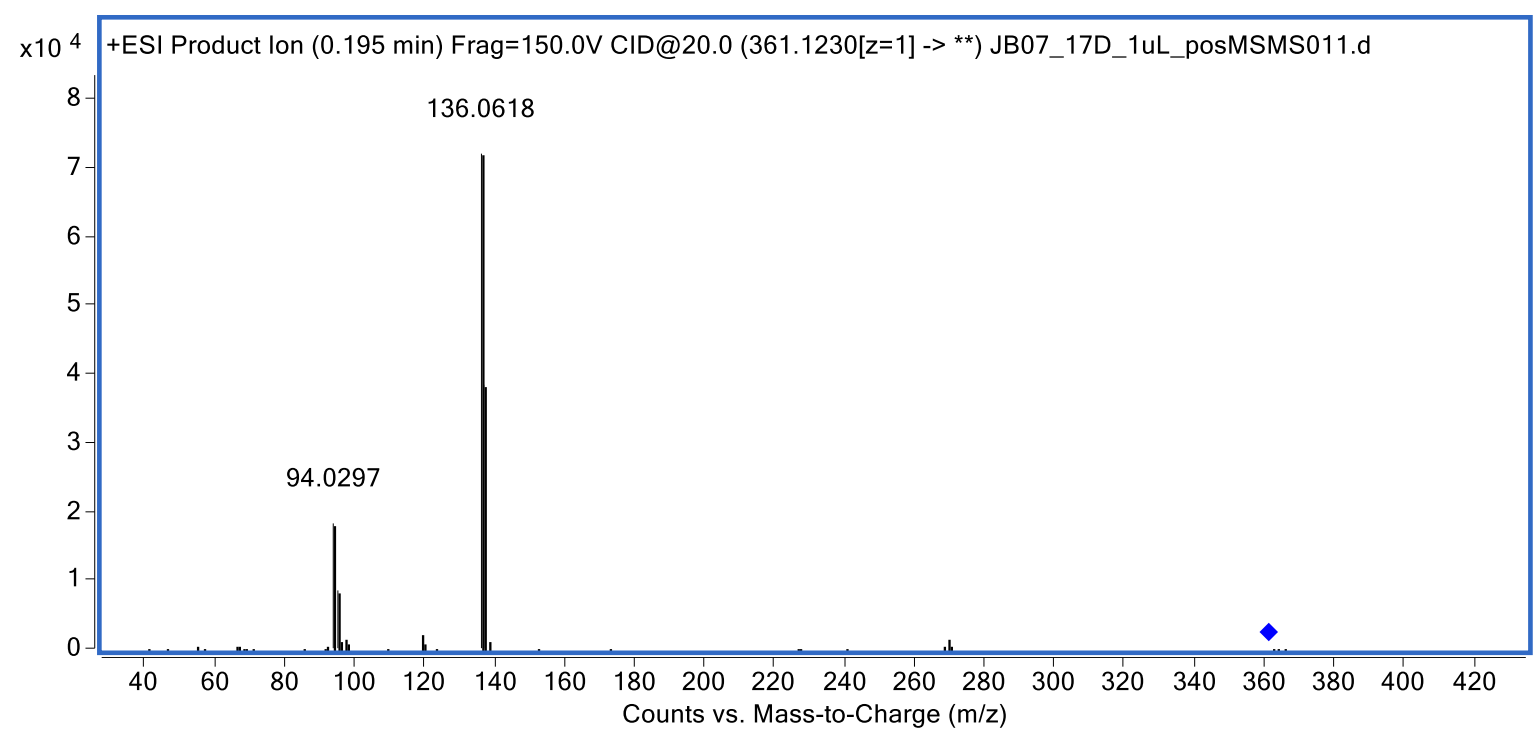

Figure S37: IR spectrum of 4

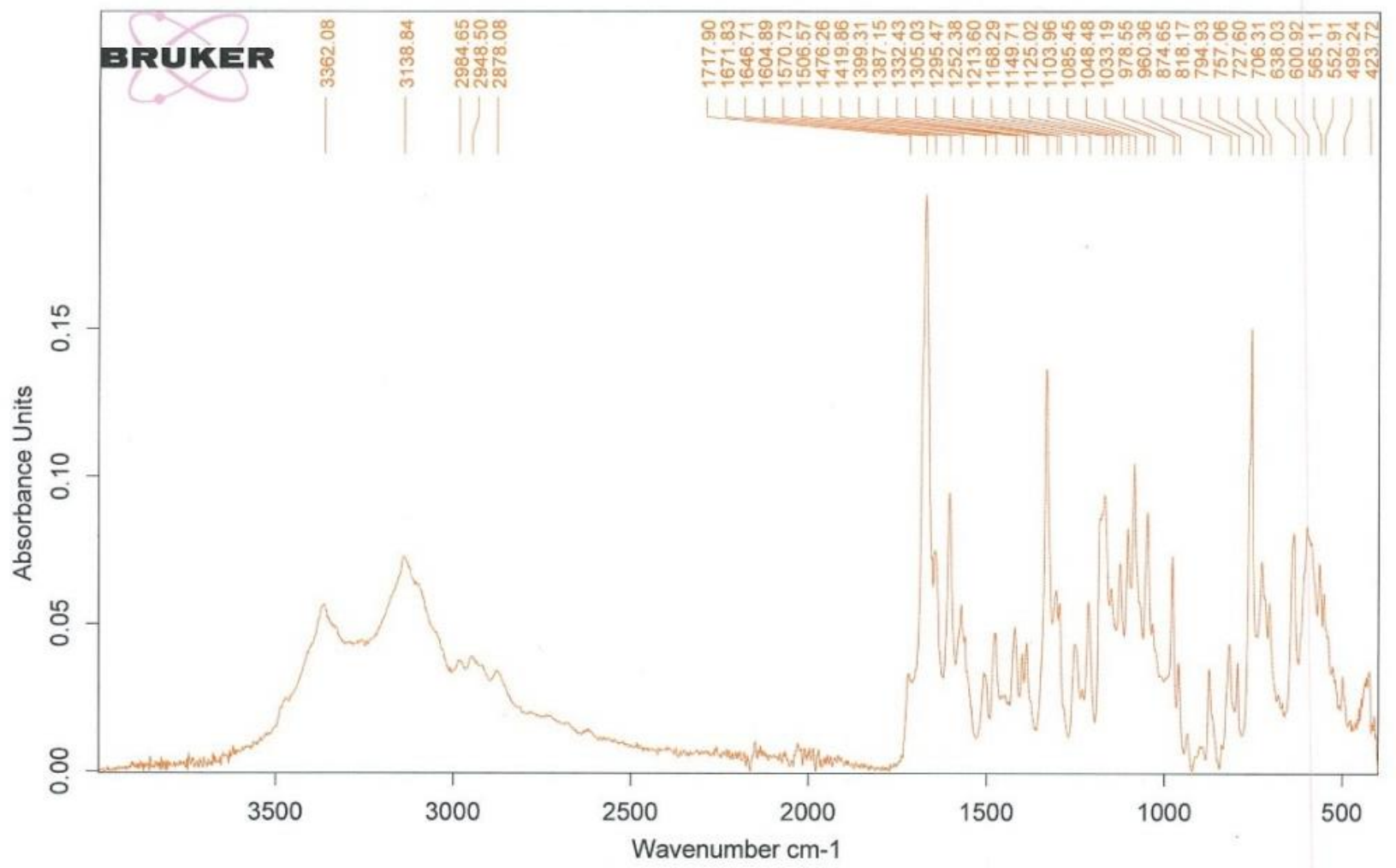


Figure S38: UV/Vis spectrum of 4

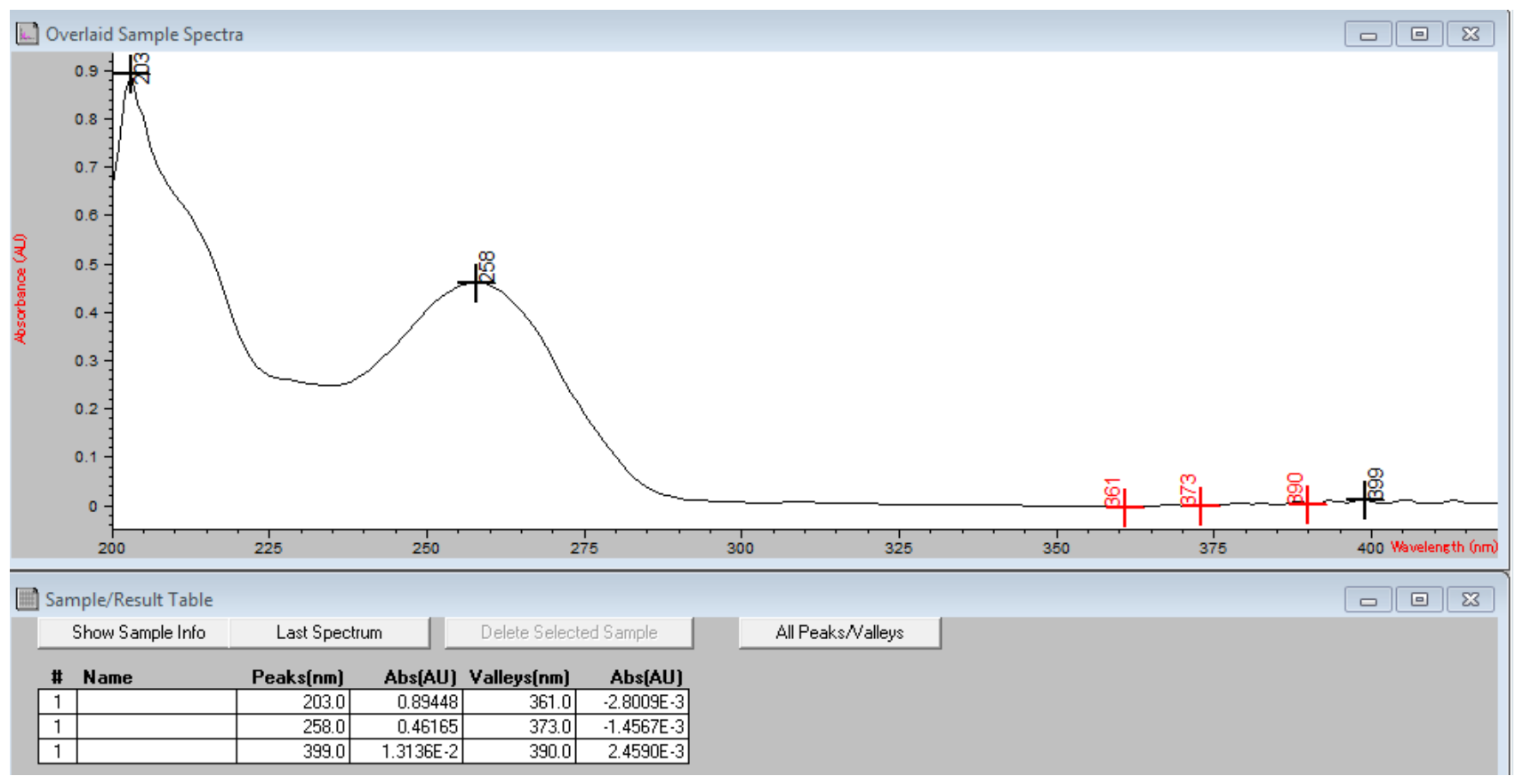


Table S7: ${ }^{13} \mathrm{C}(150 \mathrm{MHz})$ and ${ }^{1} \mathrm{H}(600 \mathrm{MHz})$ NMR data for inosyl-6'-pyrrole-3-carboxylate (5) in DMSO-d 6

\begin{tabular}{|c|c|c|c|c|}
\hline position & ${ }^{13} \mathrm{C}(\delta)$ & $\begin{array}{c}{ }^{1} \mathrm{H} \\
(\delta, \text { mult., } J \text { in } H z)\end{array}$ & COSY & HMBC \\
\hline 2 & 146 & $8.04(\mathrm{~s})$ & & 4,6 \\
\hline 4 & 148.3 & & & \\
\hline 5 & 124.5 & & & \\
\hline 6 & 156.6 & & & \\
\hline 8 & 138.6 & $8.22(\mathrm{~s})$ & & 4,5 \\
\hline $1^{\prime}$ & 87.6 & $5.90(\mathrm{~d}, 5.1)$ & $2^{\prime}$ & $4,8,2^{\prime}$ \\
\hline $2^{\prime}$ & 73.6 & $4.56(\mathrm{t}, 5.1)$ & $1^{\prime}, 3^{\prime}$ & $1^{\prime}, 4^{\prime}$ \\
\hline $3^{\prime}$ & 70.3 & $4.25(\mathrm{t}, 5.1)$ & $2^{\prime}, 4^{\prime}$ & $1^{\prime}, 5^{\prime}$ \\
\hline $4^{\prime}$ & 82.1 & $4.17(\mathrm{~m})$ & $3^{\prime}, 5^{\prime}$ & $3^{\prime}$ \\
\hline \multirow[t]{2}{*}{$5^{\prime}$} & 62.8 & $4.41(\mathrm{dd}, 12.1,3.7)$ & $4^{\prime}$ & 3', 6" \\
\hline & & $4.32(\mathrm{dd}, 12.1,5.4)$ & $4^{\prime}$ & $3^{\prime}, 4^{\prime}, 6^{\prime \prime}$ \\
\hline NH-1" & & $11.49(\mathrm{~s})$ & 2", 4", 5" & \\
\hline $2 "$ & 124.0 & $7.43(\mathrm{~m})$ & NH-1", 4", 5" & 3", 4", 5" \\
\hline $3^{\prime \prime}$ & 114.4 & & & \\
\hline 4" & 108.8 & $6.43(\mathrm{~m})$ & NH-1", 2", 5" & 2", 3", 5" \\
\hline $5^{\prime \prime}$ & 119.6 & $6.83(\mathrm{~m})$ & NH-1", 2", 4" & \\
\hline 6" & 164.0 & & & \\
\hline
\end{tabular}

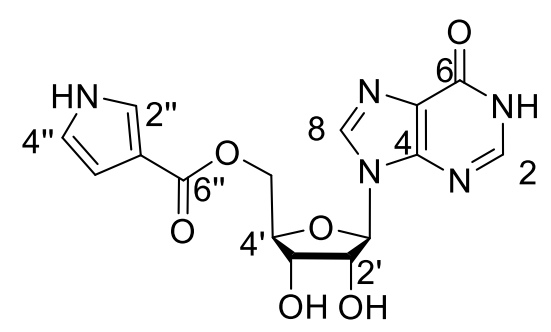

5 
Figure S39: ${ }^{1} \mathrm{H}$ NMR Spectrum (600 MHz, DMSO-d 6 ) of 5

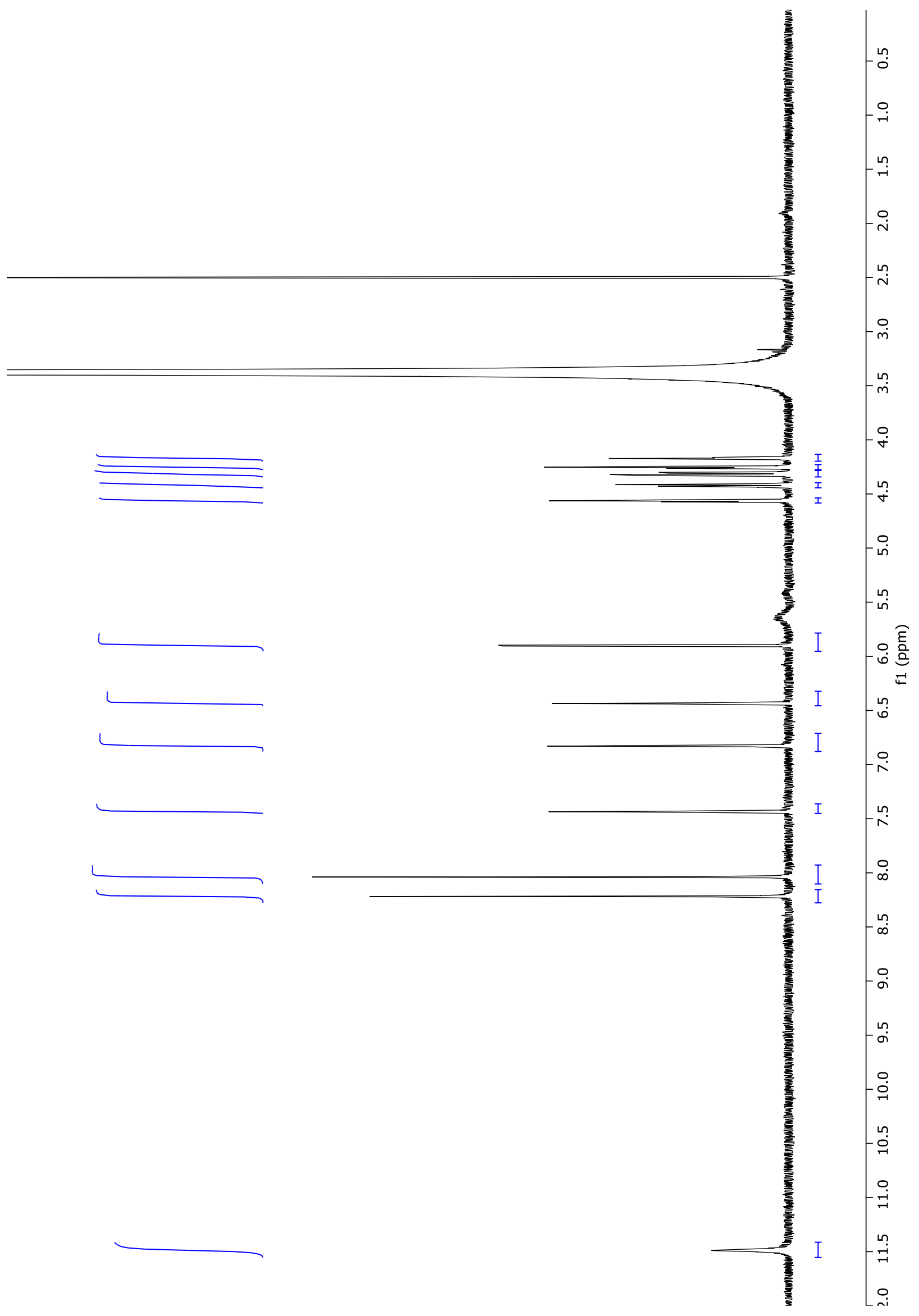


Figure S40: ${ }^{13} \mathrm{C}$ NMR Spectrum (150 MHz, DMSO-d 6 ) of 5

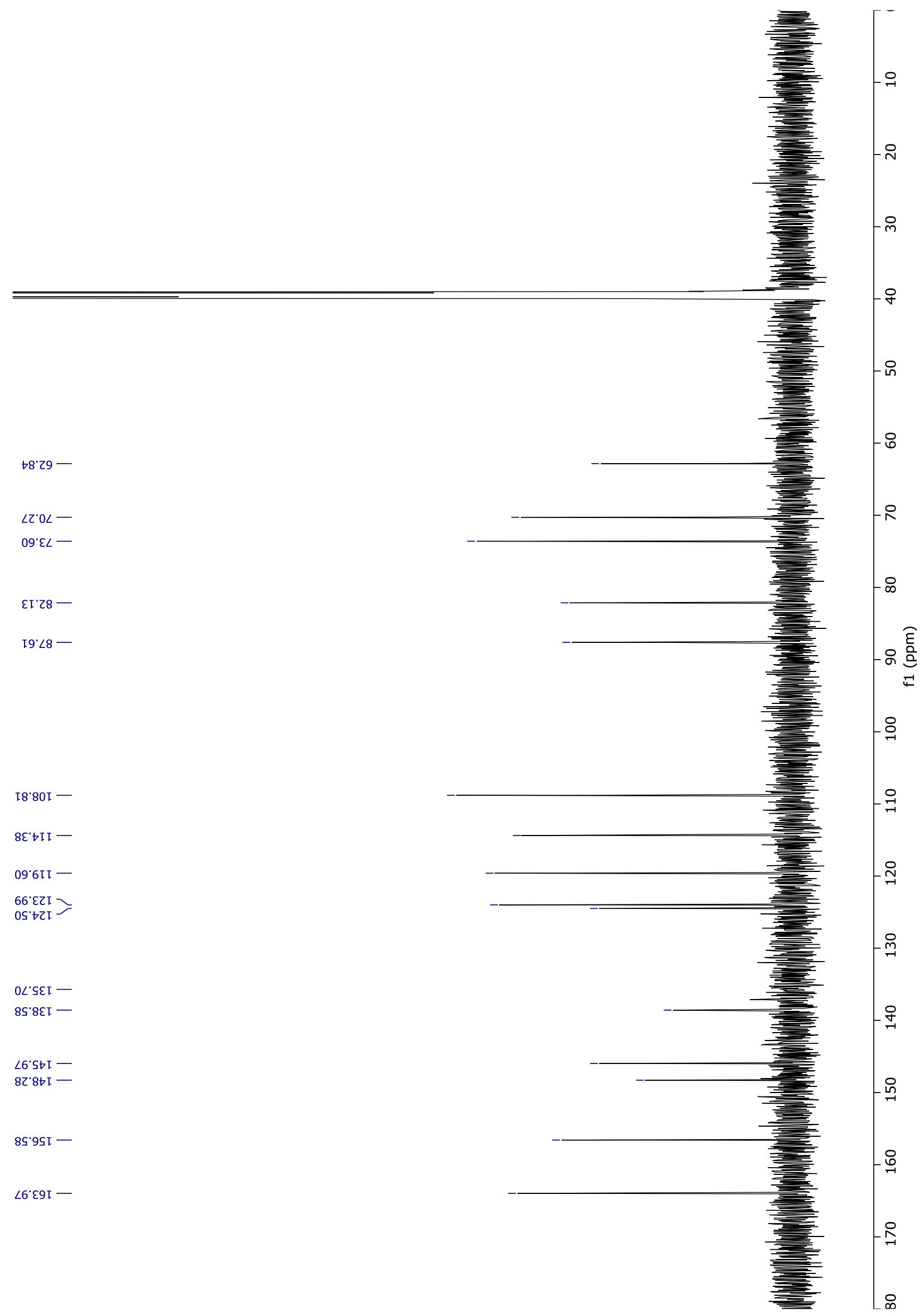


Figure S41: COSY Spectrum $\left(600 \mathrm{MHz}, \mathrm{DMSO}_{-} \mathrm{d}_{6}\right)$ of 5

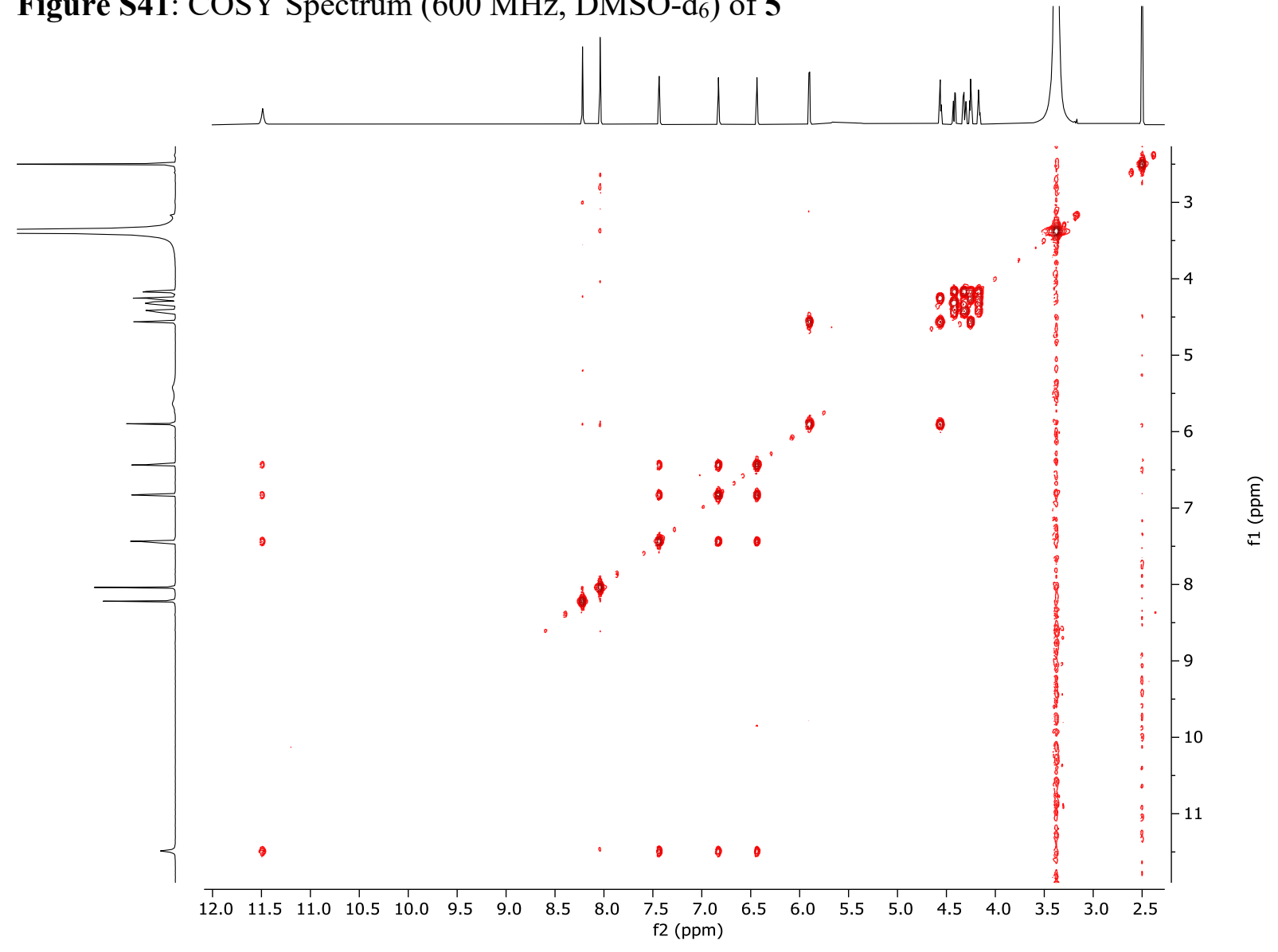

Figure S42: HSQC Spectrum (600 MHz, DMSO-d $)$ of 5

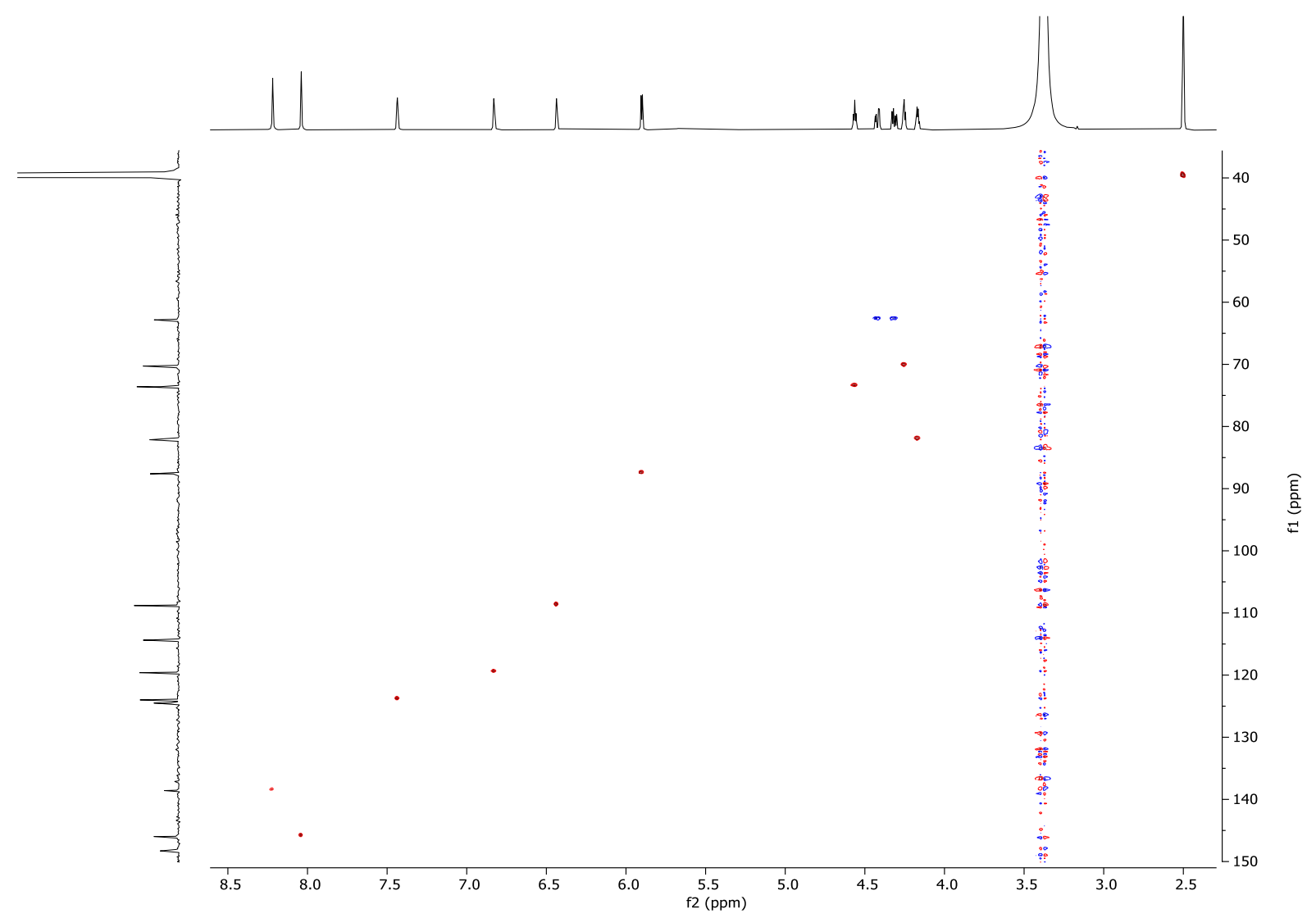


Figure S43: HMBC Spectrum (600 MHz, DMSO-d $\left.\mathrm{d}_{6}\right)$ of 5

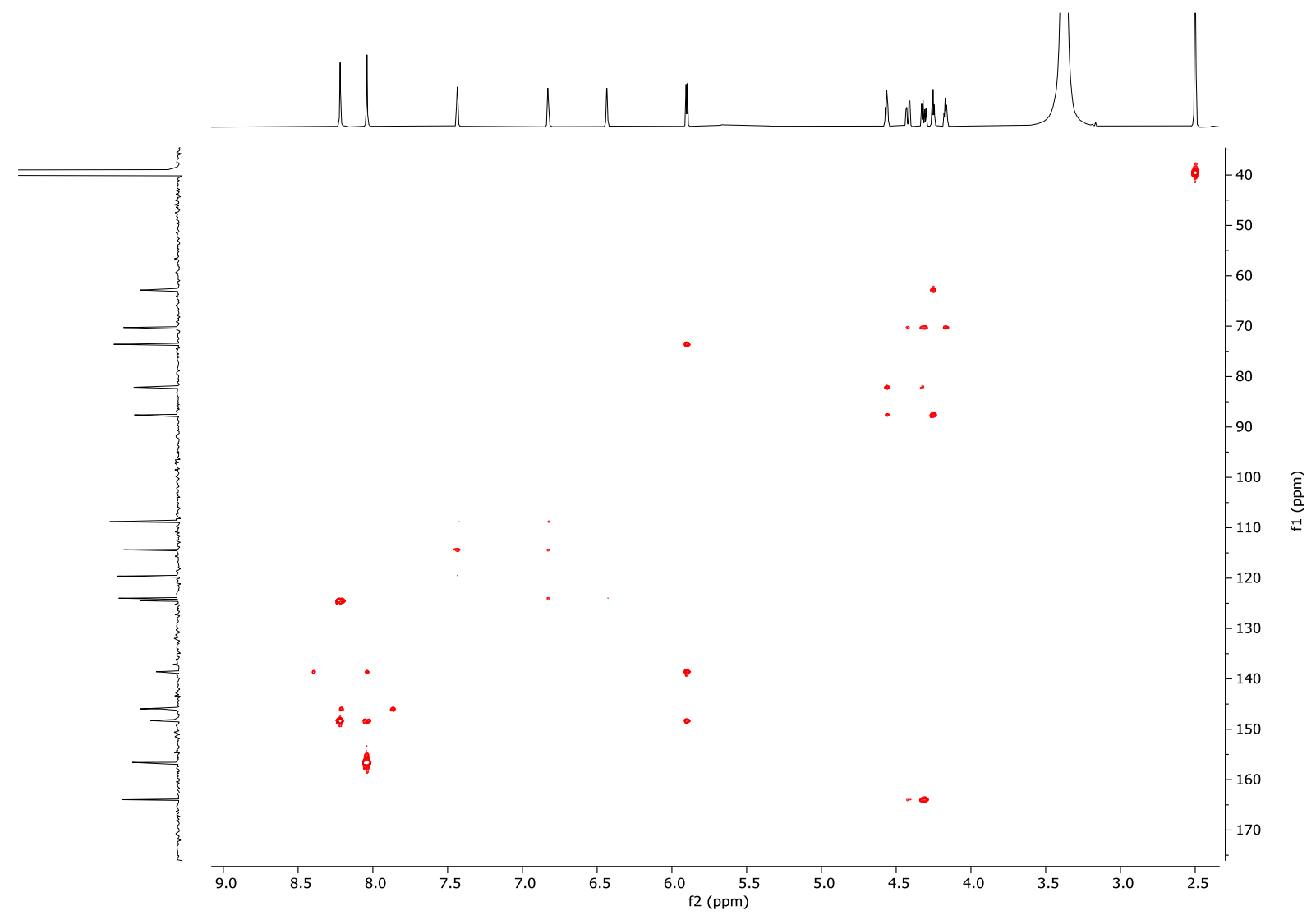

Figure S44: (+)-HRESIMS spectrum of 5

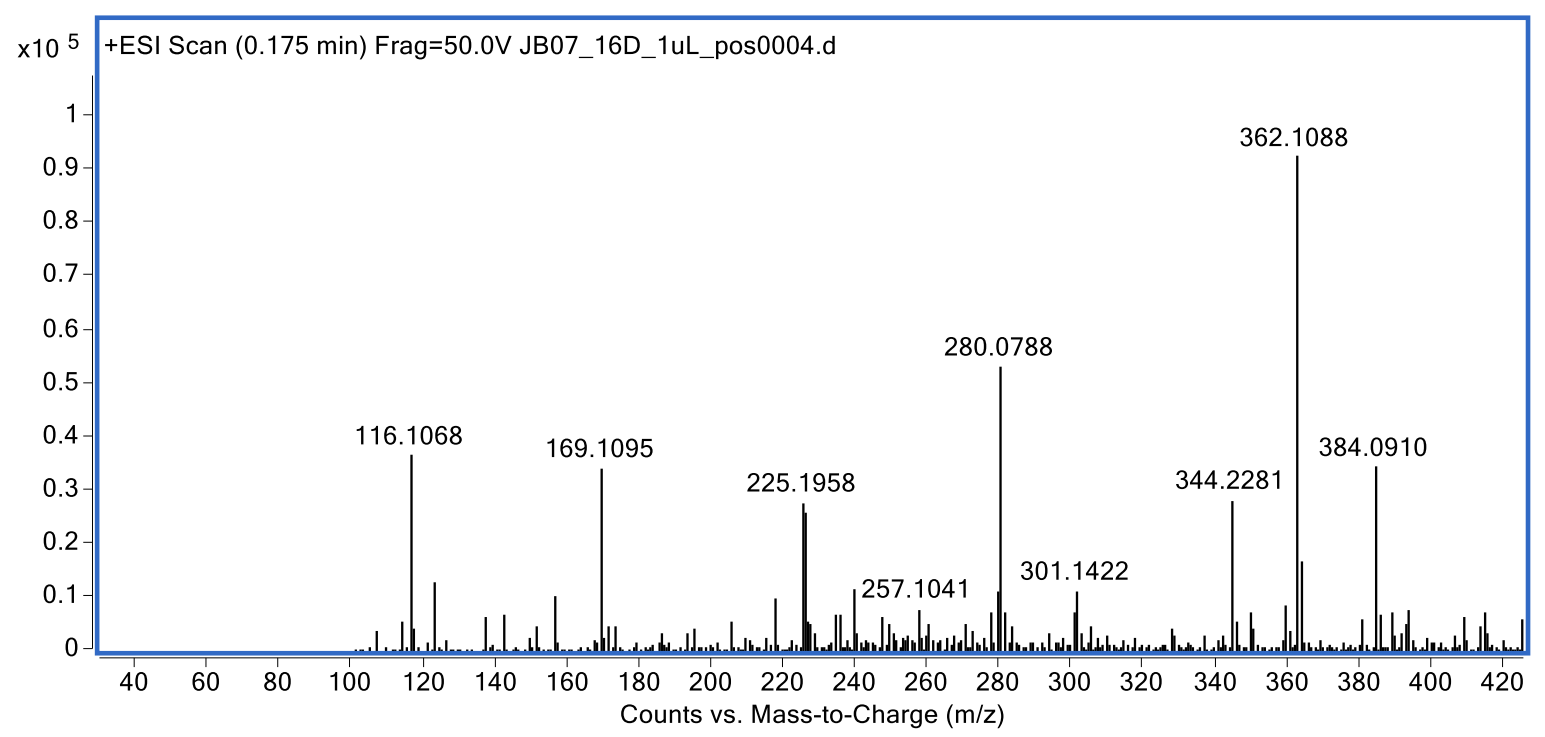


Figure S45: (+)-HRESI-MS/MS spectrum 5

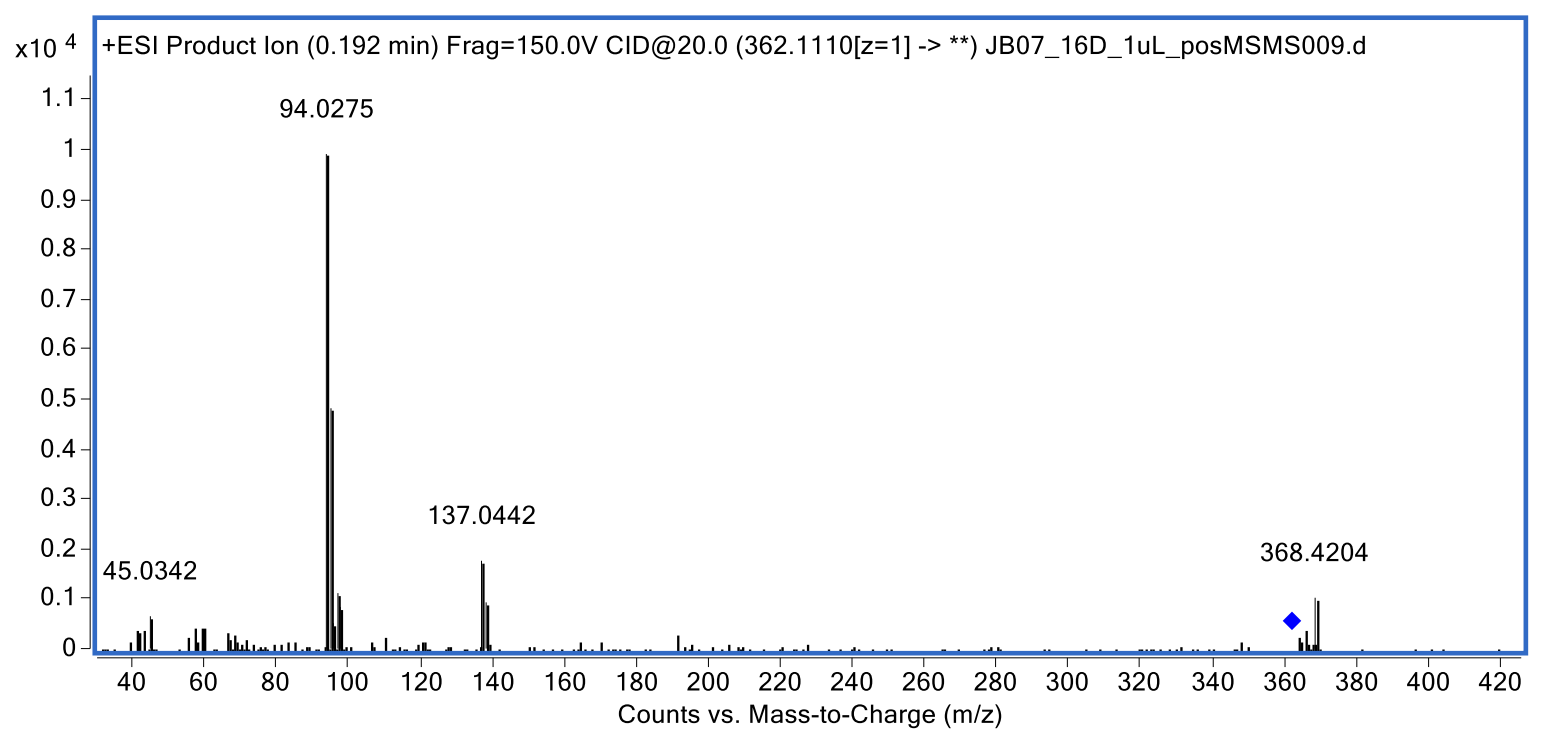

Figure S46: IR spectrum of 5

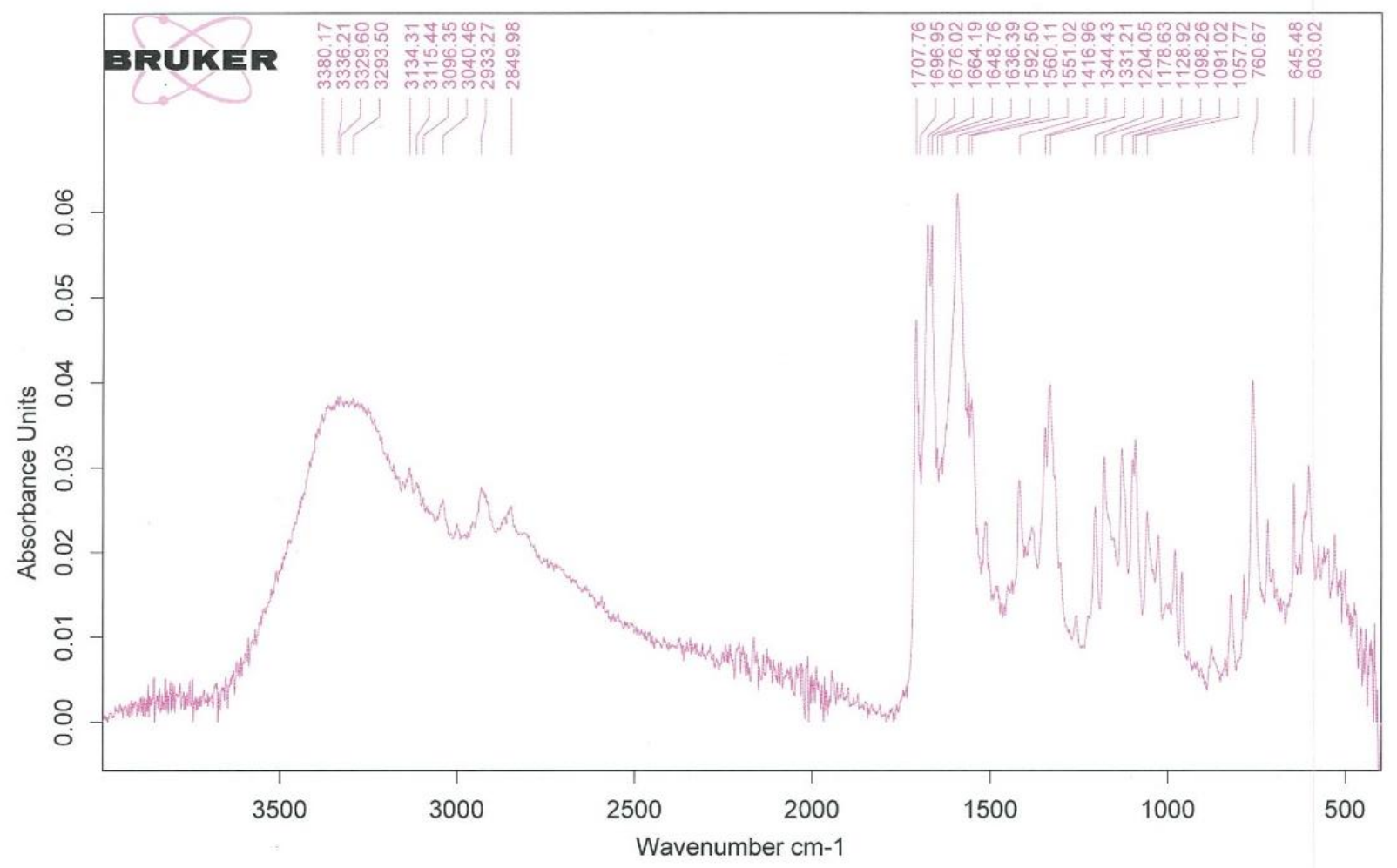


Figure S47: UV/Vis spectrum of 5

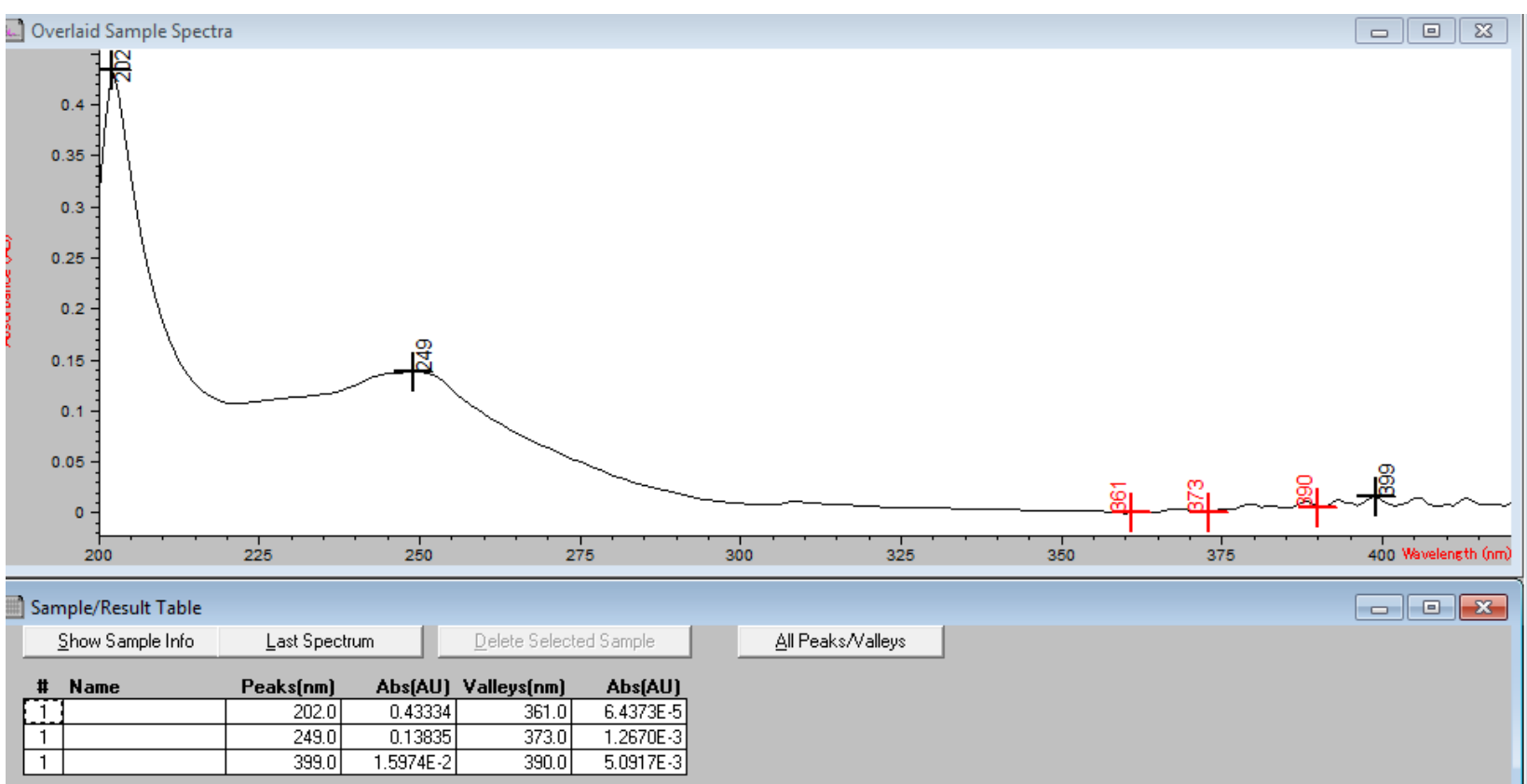


Table S8: ${ }^{13} \mathrm{C}(150 \mathrm{MHz})$ and ${ }^{1} \mathrm{H}(600 \mathrm{MHz})$ NMR data for guanosyl-6'-pyrrole-3-carboxylate (6) in DMSO-d 6

\begin{tabular}{|c|c|c|c|c|}
\hline position & ${ }^{13} \mathrm{C}(\delta)$ & $\begin{array}{c}{ }^{1} \mathrm{H} \\
(\delta, \text { mult., } J \text { in } H z)\end{array}$ & COSY & HMBC \\
\hline 2 & 153.9 & & & \\
\hline $\mathrm{NH}_{2}-2$ & & $6.52(\mathrm{~s})$ & & \\
\hline 4 & 151.5 & & & \\
\hline 5 & 116.8 & & & \\
\hline 6 & 156.9 & & & \\
\hline 8 & 135.6 & $7.77(\mathrm{~s})$ & & 4,5 \\
\hline $1^{\prime}$ & 86.6 & $5.7(\mathrm{~d}, 5.4)$ & $2^{\prime}$ & $4,8,2^{\prime}$ \\
\hline $2^{\prime}$ & 73.5 & $4.42(\mathrm{t}, 5.3)$ & $1^{\prime}, 3^{\prime}$ & \\
\hline $3^{\prime}$ & 70.5 & $4.18(\mathrm{t}, 4.8)$ & $2^{\prime}, 4^{\prime}$ & $1^{\prime}$ \\
\hline $4^{\prime}$ & 81.9 & $4.08(\mathrm{~m})$ & $3^{\prime}, 5^{\prime}$ & \\
\hline \multirow[t]{2}{*}{$5^{\prime}$} & 63.1 & $4.37(\mathrm{dd}, 12.0,3.7)$ & $4^{\prime}$ & $3^{\prime}, 6^{\prime \prime}$ \\
\hline & & $4.26(\mathrm{dd}, 12.0,5.3)$ & $4^{\prime}$ & $4^{\prime}, 6^{\prime \prime}$ \\
\hline NH-1" & & $11.46(\mathrm{~s})$ & $2^{\prime \prime}, 4^{\prime \prime}, 5^{\prime \prime}$ & \\
\hline $2^{\prime \prime}$ & 124.1 & $7.4(\mathrm{~m})$ & NH-1", 4", 5" & \\
\hline $3 "$ & 114.5 & - & & \\
\hline $4 "$ & 108.9 & $6.42(\mathrm{~m})$ & NH-1", 2", 5" & \\
\hline $5^{\prime \prime}$ & 119.8 & $6.81(\mathrm{~m})$ & NH-1", 2", 4" & \\
\hline $6^{\prime \prime}$ & 164.1 & & & \\
\hline
\end{tabular}

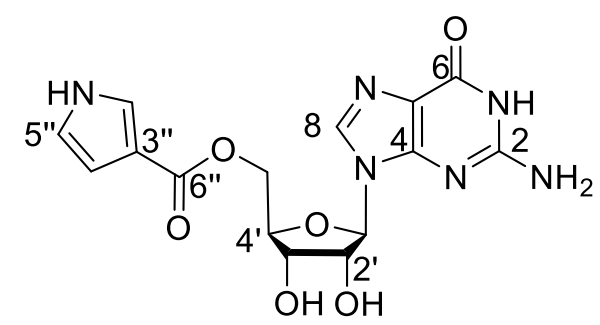

6 
Figure S48: ${ }^{1} \mathrm{H}$ NMR Spectrum (600 MHz, DMSO-d 6 ) of 6

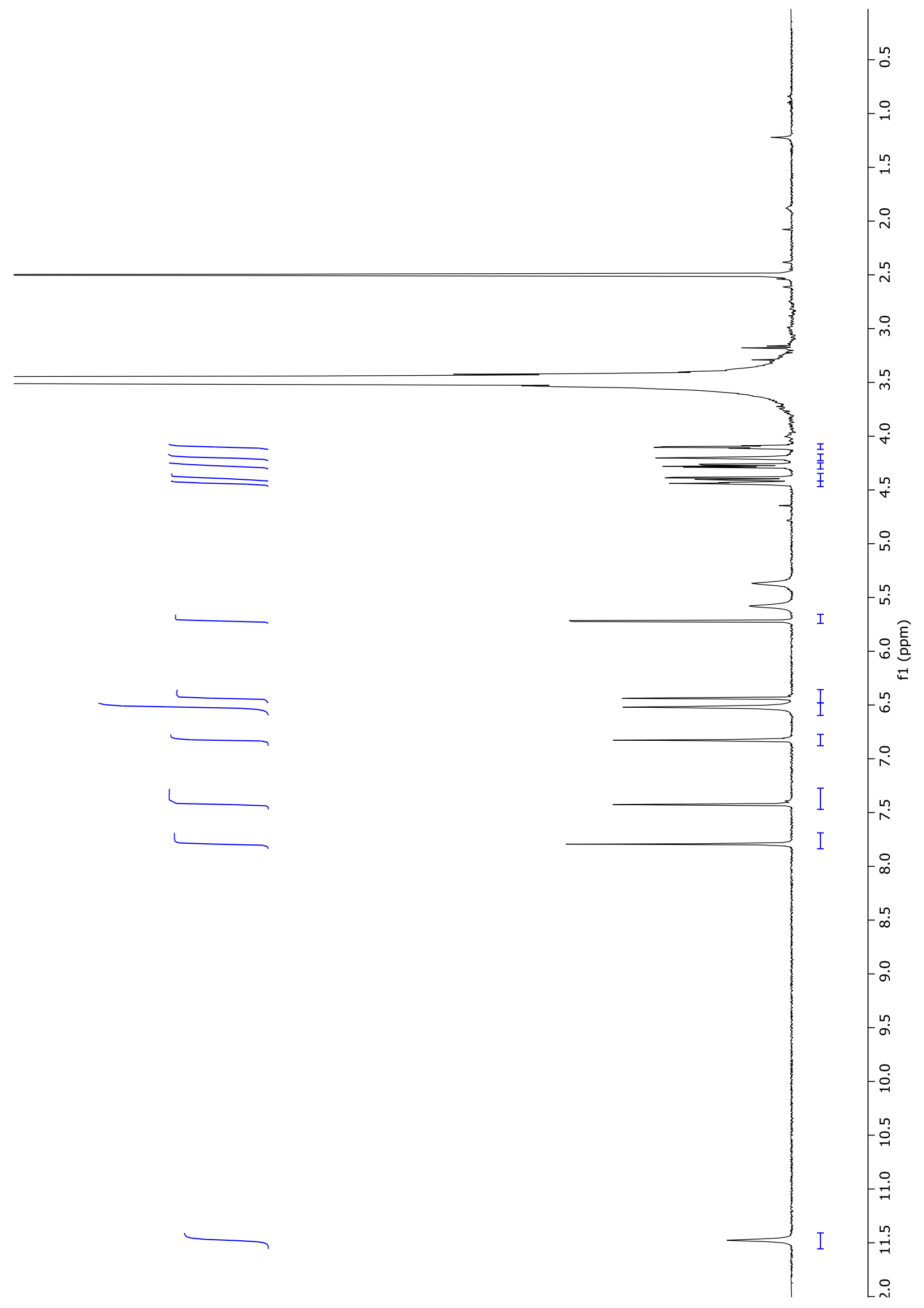


Figure S49: ${ }^{13} \mathrm{C}$ NMR Spectrum (150 MHz, DMSO-d 6 ) of 6

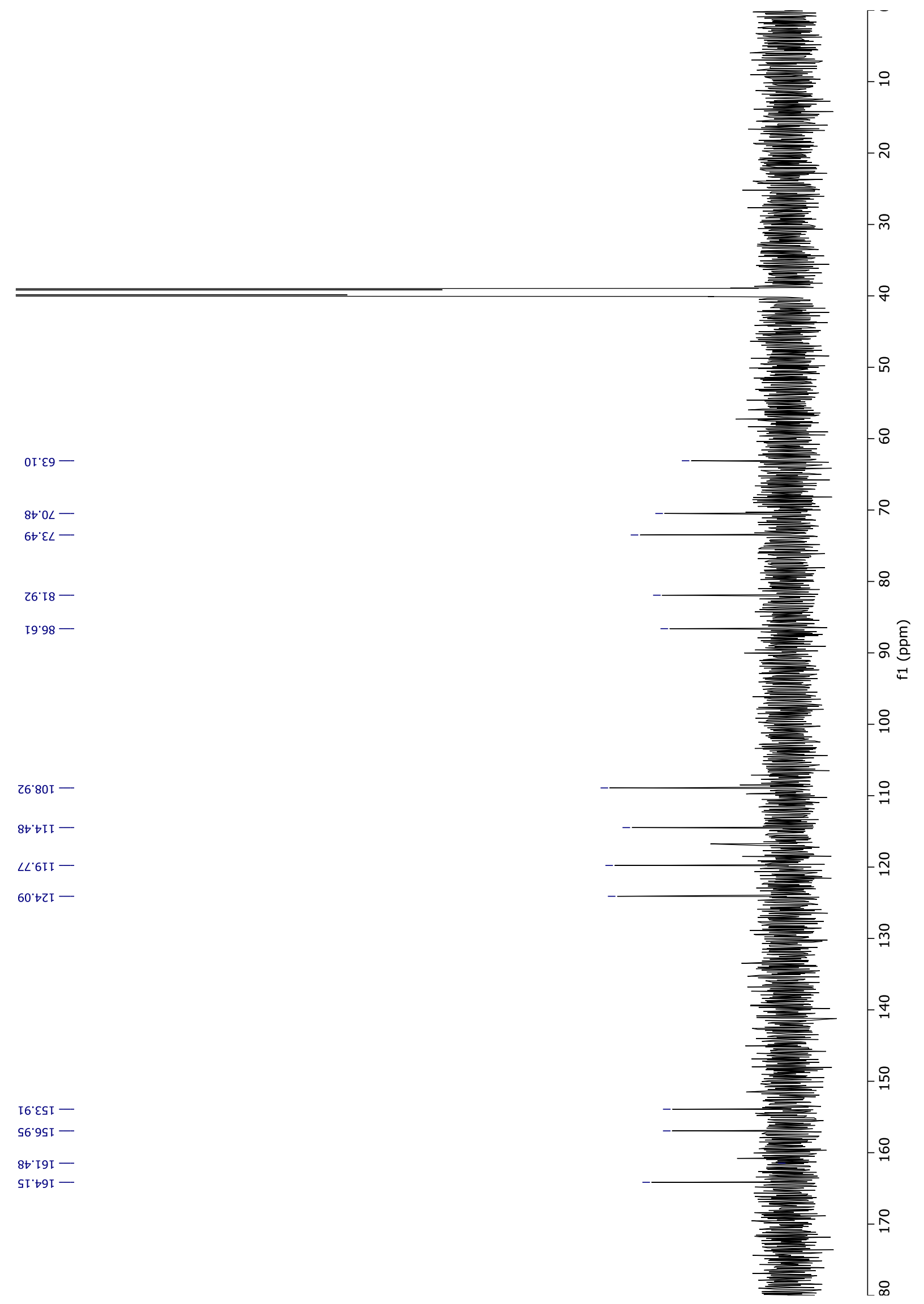


Figure S50: COSY Spectrum (600 MHz, DMSO-d $\left.{ }_{6}\right)$ of 6

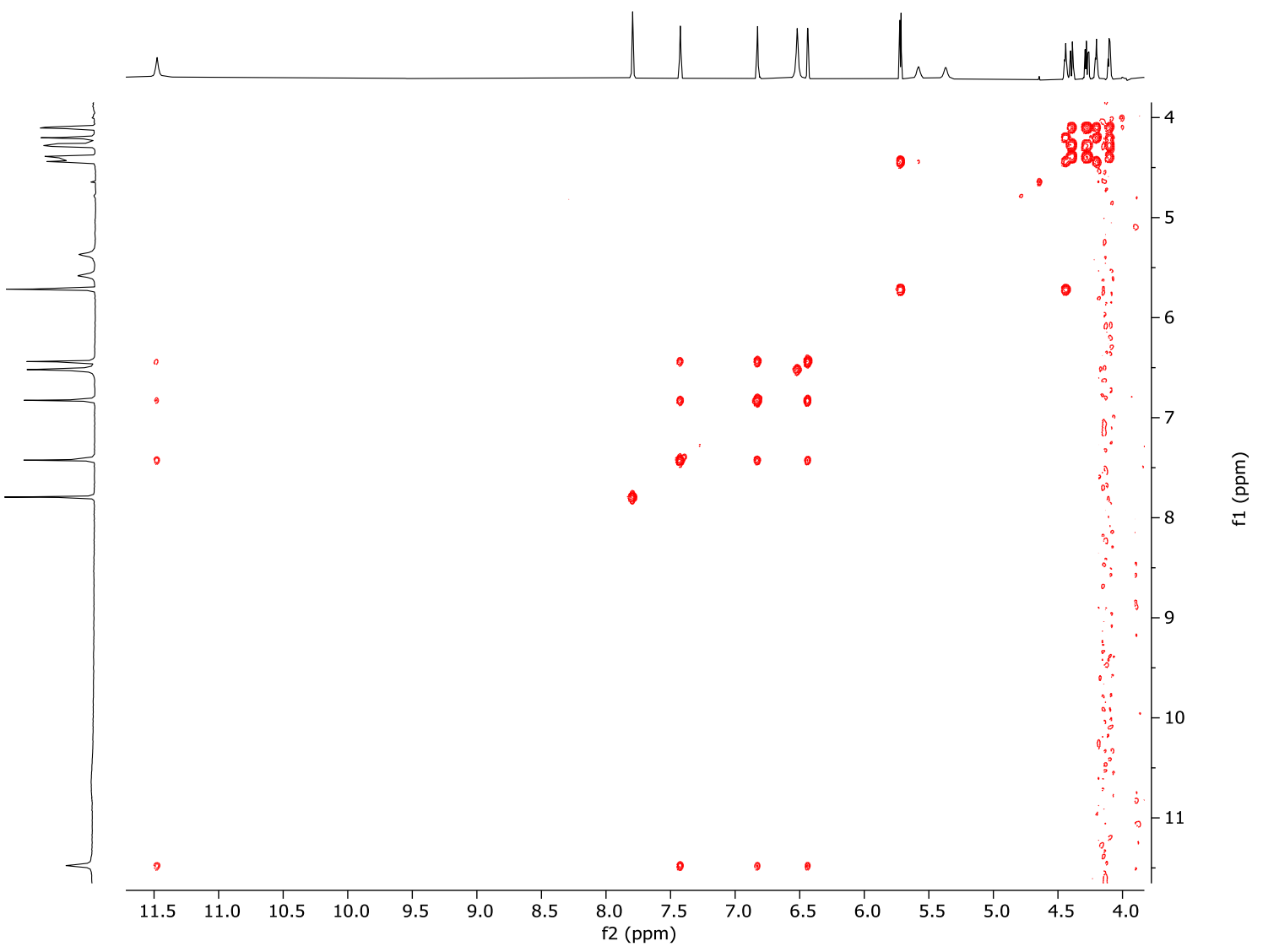

Figure S51: HSQC Spectrum (600 MHz, DMSO-d 6 ) of 6

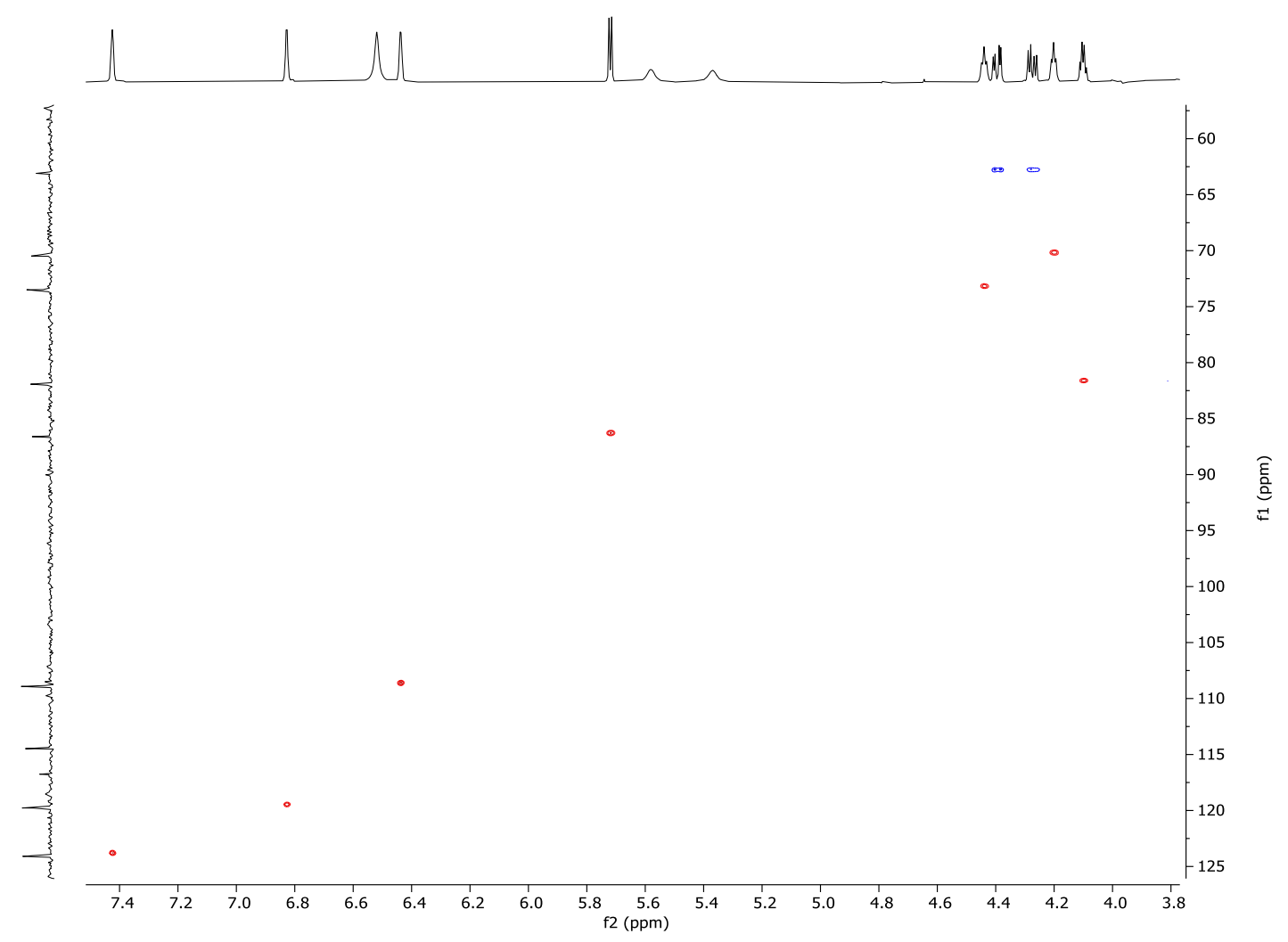


Figure S52: HMBC Spectrum (600 MHz, DMSO-d $\left.\mathrm{d}_{6}\right)$ of 6

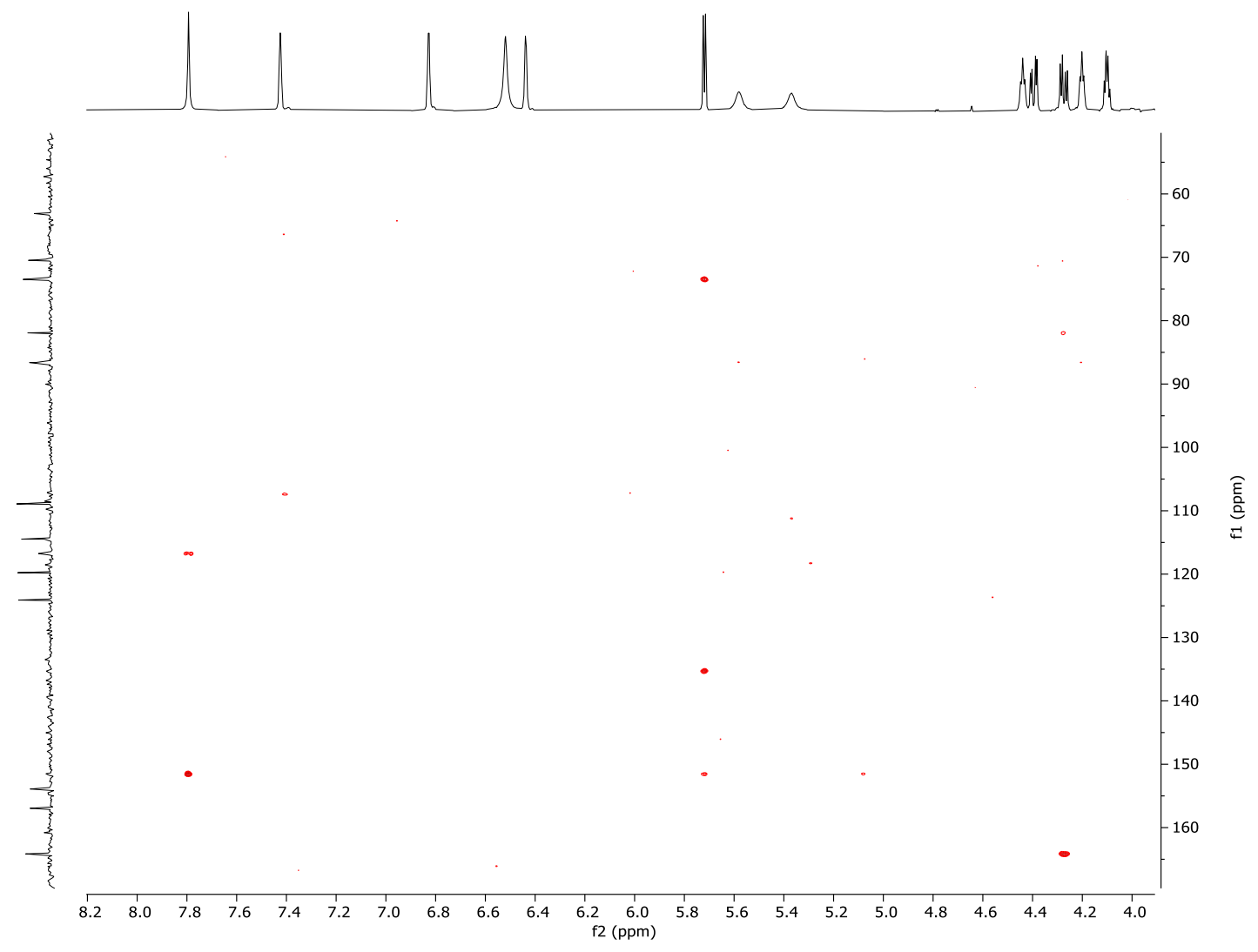

Figure S53: (+)-HRESIMS spectrum of 6

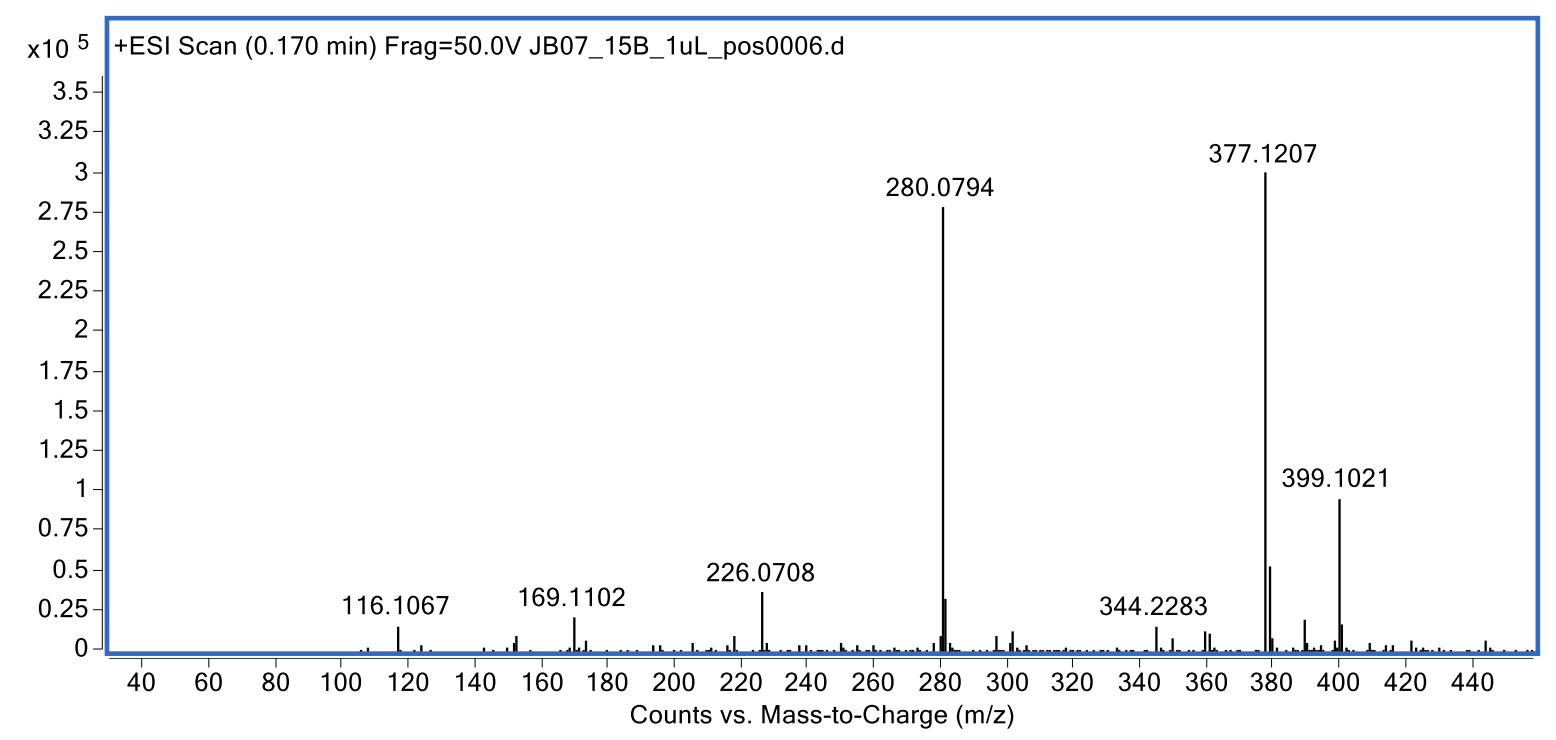


Figure S54: (+)-HRESI-MS/MS spectrum of 6

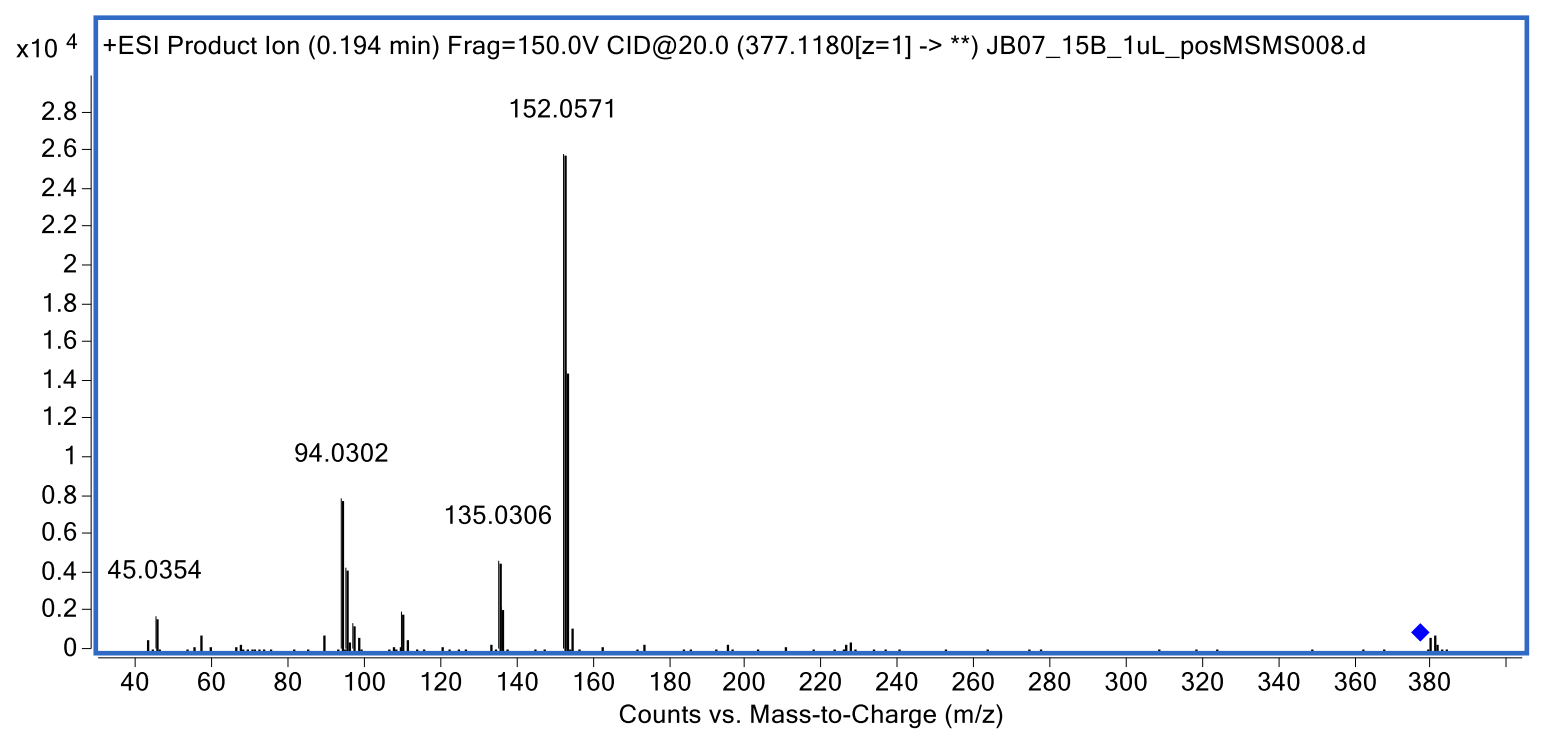

Figure S55: IR spectrum of 6

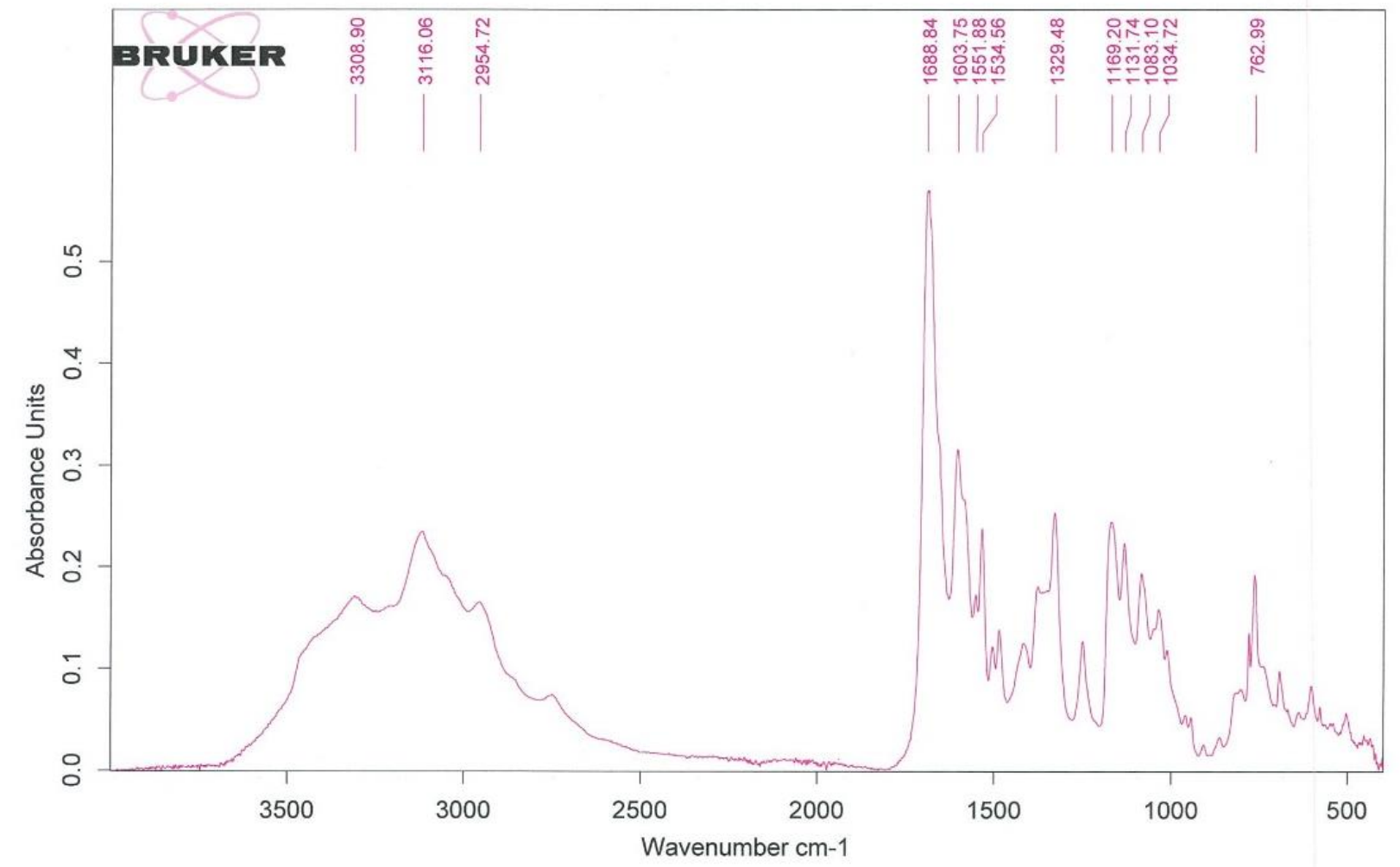


Figure S56: UV/Vis spectrum of 6

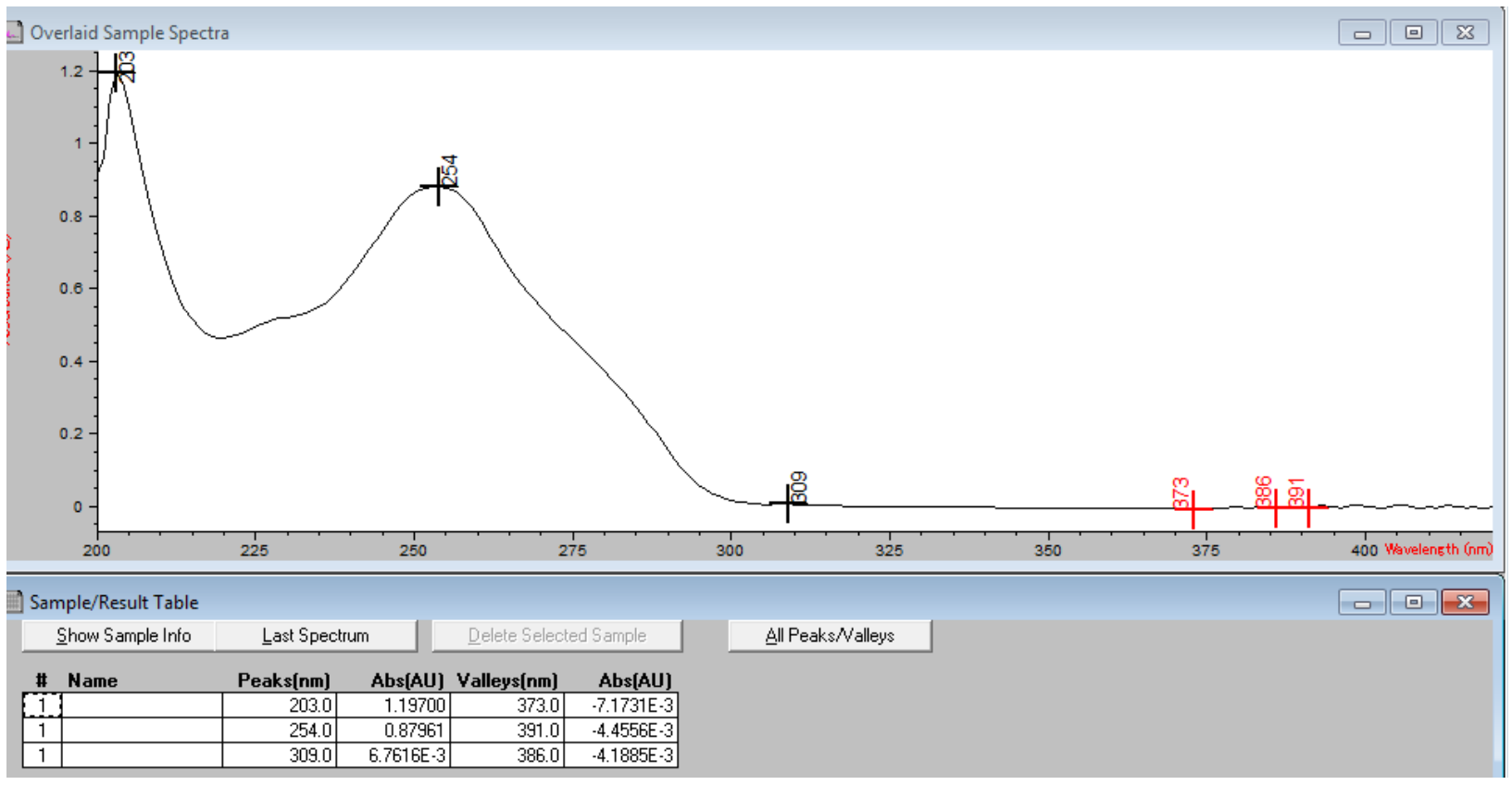


Figure S57: SEM illustration of an internode of the turfing bryozoan Nelliella nelliiformis, from Cathedral Cave Eua', Tonga. The stem is $425 \mu \mathrm{m}$ in diameter mid-length.

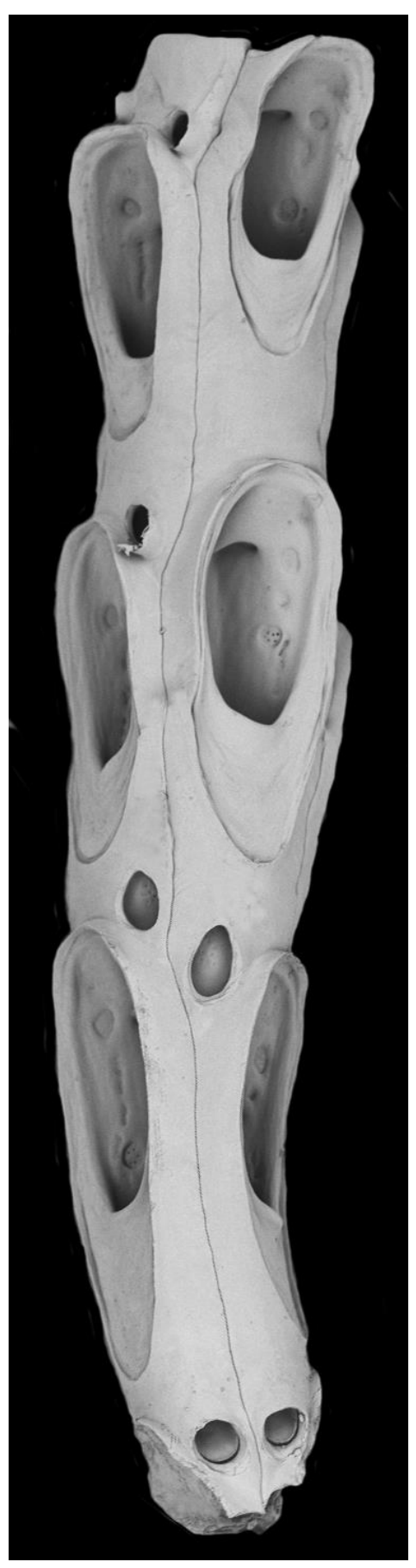


Table S9: Inhibition of 485 human-disease relevant kinases by nellielloside A (1) at $10 \mu \mathrm{M}$ (ThermoFisher Life Technologies SelectScreen ${ }^{\circledR}$ Whole Panel ACCESS Program, Wisconsin, USA. https://www.thermofisher.com/nz/en/home/products-and-services/services/customservices/screening-and-profiling-services/selectscreen-profiling-service/selectscreen-kinaseprofiling-service.html). All measurements were made using solutions of the compounds in $100 \%$ DMSO; results are quoted as percent inhibition at $10 \mu \mathrm{M}$ concentration. The Z'-LYTE®, LanthaScreen and Adapta Screening Protocols were used to obtain the results for the respective kinases as reported below. Red $=>80 \%$ inhibition, blue $<40 \%$ inhibition.

\begin{tabular}{|c|c|c|c|c|}
\hline Kinase & $\begin{array}{l}\text { Result } \\
1\end{array}$ & $\begin{array}{l}\text { Result } \\
2\end{array}$ & Mean & Assay \\
\hline GSK3B (GSK3 beta) & 100 & 100 & 100 & z-lyte \\
\hline GSK3A (GSK3 alpha) & 99 & 99 & 99 & z-lyte \\
\hline MAPKAPK3 & 98 & 99 & 98 & z-lyte \\
\hline RPS6KA3 (RSK2) & 97 & 96 & 96 & z-lyte \\
\hline MELK & 96 & 95 & 95 & z-lyte \\
\hline MAPKAPK2 & 98 & 93 & 95 & z-lyte \\
\hline MAPK14 (p38 alpha) & 95 & 95 & 95 & z-lyte \\
\hline GSG2 (Haspin) & 95 & 95 & 95 & adapta \\
\hline AKT2 (PKB beta) & 94 & 96 & 95 & z-lyte \\
\hline AKT3 (PKB gamma) & 92 & 95 & 94 & z-lyte \\
\hline CAMK1 (CaMK1) & 93 & 94 & 94 & adapta \\
\hline PLK3 & 94 & 92 & 93 & z-lyte \\
\hline GRK4 & 91 & 92 & 91 & z-lyte \\
\hline RPS6KA6 (RSK4) & 87 & 89 & 88 & z-lyte \\
\hline AKT1 (PKB alpha) & 83 & 84 & 84 & z-lyte \\
\hline ROCK2 & 81 & 80 & 80 & z-lyte \\
\hline BMPR1B (ALK6) & 79 & 77 & 78 & lantha screen \\
\hline MAP4K5 (KHS1) & 77 & 78 & 77 & z-lyte \\
\hline BMPR2 & 76 & 76 & 76 & lantha screen \\
\hline AMPK A2/B1/G1 & 74 & 76 & 75 & z-lyte \\
\hline LRRK2 G2019S & 74 & 74 & 74 & adapta \\
\hline MAPK8 (JNK1) & 74 & 71 & 72 & lantha screen \\
\hline GRK6 & 73 & 71 & 72 & z-lyte \\
\hline MAP2K4 (MEK4) & 70 & 71 & 71 & lantha screen \\
\hline SGK (SGK1) & 68 & 70 & 69 & z-lyte \\
\hline AURKA (Aurora A) & 68 & 67 & 68 & z-lyte \\
\hline CLK4 & 65 & 71 & 68 & lantha screen \\
\hline LRRK2 G2019S FL & 68 & 67 & 67 & adapta \\
\hline MAP4K4 (HGK) & 65 & 68 & 67 & z-lyte \\
\hline RPS6KA5 (MSK1) & 66 & 66 & 66 & z-lyte \\
\hline
\end{tabular}




\begin{tabular}{|c|c|c|c|c|}
\hline BLK & 65 & 66 & 66 & z-lyte \\
\hline PRKACA (PKA) & 64 & 64 & 64 & z-lyte \\
\hline $\begin{array}{l}\text { MAP2K1 (MEK1) S218D } \\
\text { S222D }\end{array}$ & 64 & 64 & 64 & lantha screen \\
\hline LRRK2 FL & 66 & 60 & 63 & adapta \\
\hline CAMK2A (CaMKII alpha) & 62 & 62 & 62 & z-lyte \\
\hline DYRK2 & 63 & 59 & 61 & lantha screen \\
\hline NLK & 59 & 60 & 60 & lantha screen \\
\hline SGK2 & 58 & 62 & 60 & z-lyte \\
\hline LRRK2 R1441C & 60 & 59 & 60 & adapta \\
\hline PRKD2 (PKD2) & 57 & 61 & 59 & z-lyte \\
\hline TGFBR2 & 61 & 58 & 59 & lantha screen \\
\hline MARK4 & 57 & 59 & 58 & z-lyte \\
\hline $\mathrm{BMX}$ & 57 & 59 & 58 & z-lyte \\
\hline LRRK2 I2020T & 58 & 57 & 58 & adapta \\
\hline AMPK (A2/B1/G2) & 57 & 58 & 58 & z-lyte \\
\hline FGR & 58 & 57 & 58 & z-lyte \\
\hline AMPK A1/B1/G1 & 57 & 58 & 57 & z-lyte \\
\hline LRRK2 & 58 & 57 & 57 & adapta \\
\hline ACVR1 (ALK2) R206H & 59 & 55 & 57 & lantha screen \\
\hline SRMS (Srm) & 57 & 57 & 57 & z-lyte \\
\hline FLT3 D835Y & 56 & 58 & 57 & z-lyte \\
\hline CAMK2B (CaMKII beta) & 57 & 56 & 57 & z-lyte \\
\hline FLT3 & 55 & 56 & 56 & z-lyte \\
\hline STK22D (TSSK1) & 57 & 53 & 55 & z-lyte \\
\hline ACVR2B & 52 & 57 & 54 & lantha screen \\
\hline MAPKAPK5 (PRAK) & 53 & 54 & 53 & z-lyte \\
\hline EGFR (ErbB1) T790M L858R & 52 & 54 & 53 & z-lyte \\
\hline RET Y791F & 53 & 52 & 53 & z-lyte \\
\hline NUAK2 & 53 & 52 & 53 & lantha screen \\
\hline ADRBK2 (GRK3) & 53 & 52 & 53 & z-lyte \\
\hline ROS1 & 52 & 52 & 52 & z-lyte \\
\hline STK17A (DRAK1) & 51 & 51 & 51 & lantha screen \\
\hline RPS6KA4 (MSK2) & 47 & 53 & 50 & z-lyte \\
\hline VRK2 & 49 & 50 & 49 & lantha screen \\
\hline ABL1 E255K & 48 & 51 & 49 & z-lyte \\
\hline ACVRL1 (ALK1) & 49 & 48 & 48 & lantha screen \\
\hline BRSK1 (SAD1) & 47 & 49 & 48 & z-lyte \\
\hline ACVR1 (ALK2) & 48 & 47 & 48 & lantha screen \\
\hline RET V804L & 48 & 47 & 48 & z-lyte \\
\hline TXK & 48 & 46 & 47 & z-lyte \\
\hline
\end{tabular}




\begin{tabular}{|c|c|c|c|c|}
\hline NEK2 & 50 & 44 & 47 & z-lyte \\
\hline CAMK2D (CaMKII delta) & 47 & 47 & 47 & z-lyte \\
\hline DDR2 T654M & 46 & 47 & 46 & lantha screen \\
\hline MLCK (MLCK2) & 45 & 47 & 46 & lantha screen \\
\hline RET & 47 & 46 & 46 & z-lyte \\
\hline MARK3 & 45 & 46 & 46 & z-lyte \\
\hline CAMK4 (CaMKIV) & 43 & 46 & 45 & z-lyte \\
\hline DYRK3 & 43 & 45 & 44 & z-lyte \\
\hline CSNK1G3 (CK1 gamma 3) & 43 & 44 & 43 & z-lyte \\
\hline ABL1 Q252H & 44 & 42 & 43 & lantha screen \\
\hline BMPR1A (ALK3) & 42 & 43 & 43 & lantha screen \\
\hline LATS2 & 43 & 41 & 42 & lantha screen \\
\hline TNK2 (ACK) & 42 & 42 & 42 & lantha screen \\
\hline CLK2 & 44 & 40 & 42 & z-lyte \\
\hline LYN B & 43 & 41 & 42 & z-lyte \\
\hline AMPK (A2/B1/G3) & 43 & 40 & 41 & z-lyte \\
\hline $\begin{array}{l}\text { MAP2K6 (MKK6) S207E } \\
\text { T211E }\end{array}$ & 40 & 42 & 41 & lantha screen \\
\hline ABL1 Y253F & 40 & 42 & 41 & z-lyte \\
\hline STK38L (NDR2) & 38 & 43 & 40 & lantha screen \\
\hline CHUK (IKK alpha) & 40 & 40 & 40 & adapta \\
\hline LCK & 39 & 41 & 40 & z-lyte \\
\hline MAPK9 (JNK2) & 42 & 37 & 40 & lantha screen \\
\hline MAP2K6 (MKK6) & 40 & 39 & 39 & z-lyte \\
\hline ABL1 M351T & 40 & 39 & 39 & lantha screen \\
\hline JAK3 & 40 & 39 & 39 & z-lyte \\
\hline EPHA4 & 40 & 39 & 39 & z-lyte \\
\hline STK23 (MSSK1) & 38 & 40 & 39 & z-lyte \\
\hline FER & 37 & 40 & 39 & z-lyte \\
\hline KDR (VEGFR2) & 37 & 40 & 39 & z-lyte \\
\hline ULK3 & 37 & 40 & 38 & lantha screen \\
\hline TGFBR1 (ALK5) & 38 & 37 & 38 & lantha screen \\
\hline CDK5/p35 & 36 & 39 & 38 & z-lyte \\
\hline CDK8/cyclin C & 35 & 40 & 37 & lantha screen \\
\hline MAPK10 (JNK3) & 36 & 37 & 37 & lantha screen \\
\hline BTK & 37 & 36 & 37 & z-lyte \\
\hline PRKD1 (PKC mu) & 38 & 34 & 36 & z-lyte \\
\hline MYLK2 (skMLCK) & 36 & 36 & 36 & z-lyte \\
\hline PRKG2 (PKG2) & 37 & 35 & 36 & z-lyte \\
\hline AMPK (A1/B2/G3) & 38 & 33 & 36 & z-lyte \\
\hline CDK11 (Inactive) & 35 & 37 & 36 & lantha screen \\
\hline
\end{tabular}




\begin{tabular}{|c|c|c|c|c|}
\hline TTK & 36 & 36 & 36 & lantha screen \\
\hline CDK11/cyclin C & 34 & 37 & 36 & lantha screen \\
\hline MAP3K19 (YSK4) & 34 & 37 & 36 & z-lyte \\
\hline KIT D816V & 31 & 40 & 36 & lantha screen \\
\hline FGFR1 V561M & 36 & 35 & 36 & lantha screen \\
\hline RET V804E & 35 & 36 & 35 & z-lyte \\
\hline NTRK3 (TRKC) & 35 & 35 & 35 & z-lyte \\
\hline ALK R1275Q & 35 & 35 & 35 & lantha screen \\
\hline STK38 (NDR) & 31 & 38 & 35 & lantha screen \\
\hline ALK C1156Y & 34 & 35 & 34 & lantha screen \\
\hline PKN2 (PRK2) & 34 & 35 & 34 & lantha screen \\
\hline TEK (TIE2) Y897S & 33 & 34 & 34 & z-lyte \\
\hline ERN2 & 36 & 31 & 33 & lantha screen \\
\hline MAP4K1 (HPK1) & 36 & 31 & 33 & lantha screen \\
\hline NEK1 & 32 & 33 & 33 & z-lyte \\
\hline ADCK3 & 33 & 32 & 33 & lantha screen \\
\hline MAP3K8 (COT) & 33 & 31 & 32 & z-lyte \\
\hline PAK4 & 30 & 34 & 32 & z-lyte \\
\hline MINK1 & 31 & 32 & 32 & z-lyte \\
\hline ALK T1151_L1152insT & 31 & 31 & 31 & lantha screen \\
\hline EPHB3 & 31 & 32 & 31 & z-lyte \\
\hline MKNK2 (MNK2) & 30 & 32 & 31 & lantha screen \\
\hline CAMK1G (CAMKI gamma) & 32 & 30 & 31 & z-lyte \\
\hline FGFR3 K650M & 32 & 30 & 31 & lantha screen \\
\hline ABL1 H396P & 30 & 32 & 31 & lantha screen \\
\hline TYRO3 (RSE) & 31 & 31 & 31 & z-lyte \\
\hline NTRK1 (TRKA) & 31 & 31 & 31 & z-lyte \\
\hline RPS6KB1 (p70S6K) & 31 & 30 & 31 & z-lyte \\
\hline PRKACB (PRKAC beta) & 29 & 32 & 31 & lantha screen \\
\hline IRAK1 & 29 & 32 & 30 & adapta \\
\hline ALK F1174L & 32 & 29 & 30 & lantha screen \\
\hline FYN & 31 & 30 & 30 & z-lyte \\
\hline MAP2K6 (MKK6) & 31 & 29 & 30 & lantha screen \\
\hline RET S891A & 29 & 31 & 30 & z-lyte \\
\hline DDR2 N456S & 28 & 32 & 30 & lantha screen \\
\hline ABL1 & 30 & 30 & 30 & z-lyte \\
\hline STK24 (MST3) & 28 & 31 & 29 & z-lyte \\
\hline NTRK2 (TRKB) & 27 & 31 & 29 & z-lyte \\
\hline FGFR1 & 28 & 30 & 29 & z-lyte \\
\hline ABL1 G250E & 27 & 31 & 29 & z-lyte \\
\hline PRKCQ (PKC theta) & 27 & 30 & 29 & z-lyte \\
\hline
\end{tabular}




\begin{tabular}{|c|c|c|c|c|}
\hline ABL1 T315I & 29 & 29 & 29 & z-lyte \\
\hline MAPK1 (ERK2) & 30 & 27 & 29 & z-lyte \\
\hline GAK & 32 & 25 & 28 & lantha screen \\
\hline MAP3K14 (NIK) & 30 & 26 & 28 & lantha screen \\
\hline PRKCG (PKC gamma) & 28 & 27 & 28 & z-lyte \\
\hline CAMK2G (CaMKII gamma) & 28 & 27 & 27 & lantha screen \\
\hline PLK1 & 28 & 27 & 27 & z-lyte \\
\hline CHEK1 (CHK1) & 30 & 24 & 27 & z-lyte \\
\hline SNF1LK2 & 26 & 28 & 27 & z-lyte \\
\hline KSR2 & 27 & 27 & 27 & z-lyte \\
\hline MAP2K1 (MEK1) & 27 & 27 & 27 & z-lyte \\
\hline BRAF & 26 & 28 & 27 & z-lyte \\
\hline CAMKK2 (CaMKK beta) & 26 & 27 & 27 & lantha screen \\
\hline YES1 & 26 & 27 & 27 & z-lyte \\
\hline EPHA1 & 27 & 26 & 26 & z-lyte \\
\hline ALK & 26 & 26 & 26 & z-lyte \\
\hline PRKG1 & 26 & 26 & 26 & z-lyte \\
\hline NUAK1 (ARK5) & 28 & 23 & 26 & adapta \\
\hline MAP3K10 (MLK2) & 24 & 27 & 25 & lantha screen \\
\hline EPHB4 & 26 & 25 & 25 & z-lyte \\
\hline ADRBK1 (GRK2) & 24 & 26 & 25 & z-lyte \\
\hline LYN A & 24 & 26 & 25 & z-lyte \\
\hline CSNK1G2 (CK1 gamma 2) & 25 & 25 & 25 & z-lyte \\
\hline CHEK2 (CHK2) & 25 & 24 & 25 & z-lyte \\
\hline ALK L1196M & 26 & 24 & 25 & lantha screen \\
\hline ROCK1 & 25 & 25 & 25 & z-lyte \\
\hline $\begin{array}{l}\text { PIK3CD/PIK3R1 (p110 } \\
\text { delta/p85 alpha) }\end{array}$ & 33 & 17 & 25 & adapta \\
\hline CSNK1D (CK1 delta) & 26 & 23 & 24 & z-lyte \\
\hline ACVR2A & 24 & 25 & 24 & lantha screen \\
\hline MAP4K2 (GCK) & 25 & 24 & 24 & z-lyte \\
\hline BRAF V599E & 25 & 23 & 24 & z-lyte \\
\hline CDK2/cyclin E1 & 25 & 22 & 24 & lantha screen \\
\hline WEE1 & 27 & 21 & 24 & lantha screen \\
\hline AMPK (A1/B2/G2) & 23 & 24 & 24 & z-lyte \\
\hline STK22B (TSSK2) & 25 & 22 & 23 & z-lyte \\
\hline EGFR (ErbB1) d747-749 A750P & 23 & 24 & 23 & lantha screen \\
\hline FES (FPS) & 22 & 24 & 23 & z-lyte \\
\hline MAP2K1 (MEK1) & 22 & 24 & 23 & lantha screen \\
\hline EPHA7 & 16 & 30 & 23 & lantha screen \\
\hline MAP3K9 (MLK1) & 19 & 27 & 23 & z-lyte \\
\hline
\end{tabular}




\begin{tabular}{|c|c|c|c|c|}
\hline RPS6KA1 (RSK1) & 23 & 22 & 23 & z-lyte \\
\hline JAK2 & 23 & 23 & 23 & z-lyte \\
\hline RAF1 (cRAF) Y340D Y341D & 22 & 23 & 23 & z-lyte \\
\hline AURKB (Aurora B) & 25 & 20 & 23 & z-lyte \\
\hline MYLK4 & 26 & 19 & 22 & lantha screen \\
\hline AMPK (A1/B1/G2) & 22 & 23 & 22 & lantha screen \\
\hline ABL2 (Arg) & 22 & 23 & 22 & z-lyte \\
\hline CDK5/p25 & 22 & 23 & 22 & z-lyte \\
\hline MET (cMet) & 20 & 25 & 22 & z-lyte \\
\hline RET A883F & 21 & 23 & 22 & z-lyte \\
\hline SPHK1 & 19 & 25 & 22 & adapta \\
\hline MAPK8 (JNK1) & 22 & 22 & 22 & z-lyte \\
\hline CDK5 (Inactive) & 18 & 25 & 21 & lantha screen \\
\hline MUSK & 26 & 16 & 21 & z-lyte \\
\hline PLK4 & 21 & 22 & 21 & lantha screen \\
\hline STK32C (YANK3) & 22 & 21 & 21 & lantha screen \\
\hline MAP2K2 (MEK2) & 22 & 20 & 21 & z-lyte \\
\hline PAK7 (KIAA1264) & 19 & 23 & 21 & z-lyte \\
\hline RPS6KB2 (p70S6Kb) & 20 & 22 & 21 & z-lyte \\
\hline CSNK1G1 (CK1 gamma 1) & 20 & 22 & 21 & z-lyte \\
\hline PRKCH (PKC eta) & 18 & 23 & 20 & z-lyte \\
\hline EGFR (ErbB1) T790M & 21 & 20 & 20 & z-lyte \\
\hline KIT A829P & 18 & 22 & 20 & lantha screen \\
\hline PRKCA (PKC alpha) & 20 & 20 & 20 & z-lyte \\
\hline $\begin{array}{l}\text { PIK3CA E542K/PIK3R1 (p110 } \\
\text { alpha E542K/p85 alpha) }\end{array}$ & 20 & 20 & 20 & adapta \\
\hline EIF2AK2 (PKR) & 20 & 20 & 20 & lantha screen \\
\hline $\operatorname{AMPK}(\mathrm{A} 1 / \mathrm{B} 1 / \mathrm{G} 3)$ & 20 & 20 & 20 & lantha screen \\
\hline TEK (Tie2) & 31 & 9 & 20 & z-lyte \\
\hline PAK6 & 20 & 20 & 20 & z-lyte \\
\hline $\mathrm{HCK}$ & 19 & 20 & 20 & z-lyte \\
\hline KIT D816H & 18 & 21 & 20 & lantha screen \\
\hline PRKCB2 (PKC beta II) & 19 & 20 & 19 & z-lyte \\
\hline CAMK1D (CaMKI delta) & 20 & 19 & 19 & z-lyte \\
\hline CDK7/cyclin H/MNAT1 & 12 & 27 & 19 & adapta \\
\hline PAK2 (PAK65) & 22 & 17 & 19 & z-lyte \\
\hline RPS6KA2 (RSK3) & 18 & 20 & 19 & z-lyte \\
\hline SLK & 19 & 19 & 19 & lantha screen \\
\hline STK32B (YANK2) & 20 & 17 & 19 & lantha screen \\
\hline FGFR2 & 23 & 14 & 19 & z-lyte \\
\hline MET M1250T & 18 & 19 & 19 & z-lyte \\
\hline
\end{tabular}




\begin{tabular}{|c|c|c|c|c|}
\hline ANKK1 & 15 & 22 & 19 & lantha screen \\
\hline PRKX & 18 & 19 & 19 & z-lyte \\
\hline CDK2/cyclin A1 & 17 & 20 & 18 & lantha screen \\
\hline MARK2 & 18 & 18 & 18 & z-lyte \\
\hline PTK2 (FAK) & 19 & 17 & 18 & z-lyte \\
\hline CDK2/cyclin A & 17 & 19 & 18 & z-lyte \\
\hline TYK2 & 18 & 18 & 18 & z-lyte \\
\hline NEK6 & 20 & 16 & 18 & z-lyte \\
\hline TEC & 16 & 20 & 18 & lantha screen \\
\hline RET M918T & 17 & 19 & 18 & lantha screen \\
\hline JAK2 JH1 JH2 & 18 & 18 & 18 & z-lyte \\
\hline PEAK1 & 16 & 19 & 18 & z-lyte \\
\hline STK17B (DRAK2) & 22 & 13 & 18 & lantha screen \\
\hline MAP2K2 (MEK2) & 17 & 18 & 17 & lantha screen \\
\hline $\begin{array}{l}\text { PIK3CA/PIK3R1 (p110 } \\
\text { alpha/p85 alpha) }\end{array}$ & 18 & 17 & 17 & adapta \\
\hline SPHK2 & 15 & 19 & 17 & adapta \\
\hline MAP3K11 (MLK3) & 18 & 16 & 17 & lantha screen \\
\hline MARK1 (MARK) & 16 & 18 & 17 & z-lyte \\
\hline DYRK1B & 18 & 16 & 17 & z-lyte \\
\hline EPHA2 & 17 & 17 & 17 & z-lyte \\
\hline SGKL (SGK3) & 16 & 18 & 17 & z-lyte \\
\hline STK39 (STLK3) & 19 & 14 & 17 & lantha screen \\
\hline PRKCN (PKD3) & 16 & 17 & 16 & z-lyte \\
\hline FGFR3 G697C & 11 & 22 & 16 & lantha screen \\
\hline CDK3/cyclin E1 & 13 & 19 & 16 & lantha screen \\
\hline KIT V559D T670I & 19 & 14 & 16 & lantha screen \\
\hline CDC7/DBF4 & 15 & 17 & 16 & lantha screen \\
\hline AAK1 & 15 & 17 & 16 & lantha screen \\
\hline DAPK2 & 17 & 14 & 16 & lantha screen \\
\hline MST4 & 15 & 16 & 16 & z-lyte \\
\hline CSNK1E (CK1 epsilon) & 14 & 17 & 16 & z-lyte \\
\hline PRKACG (PRKAC gamma) & 14 & 16 & 15 & lantha screen \\
\hline MAPK7 (ERK5) & 14 & 16 & 15 & z-lyte \\
\hline TLK2 & 15 & 15 & 15 & lantha screen \\
\hline RET G691S & 16 & 14 & 15 & lantha screen \\
\hline IRAK3 & 14 & 15 & 15 & lantha screen \\
\hline HIPK4 & 15 & 15 & 15 & z-lyte \\
\hline MYO3B (MYO3 beta) & 18 & 11 & 15 & lantha screen \\
\hline $\begin{array}{l}\text { MAP3K7/MAP3K7IP1 (TAK1- } \\
\text { TAB1) }\end{array}$ & 15 & 15 & 15 & lantha screen \\
\hline
\end{tabular}




\begin{tabular}{|c|c|c|c|c|}
\hline MYLK (MLCK) & 14 & 15 & 15 & lantha screen \\
\hline CSNK1E (CK1 epsilon) R178C & 16 & 14 & 15 & z-lyte \\
\hline MERTK (cMER) A708S & 15 & 14 & 15 & lantha screen \\
\hline ULK1 & 13 & 15 & 14 & lantha screen \\
\hline TAOK3 (JIK) & 16 & 12 & 14 & lantha screen \\
\hline CDK13/cyclin K & 11 & 18 & 14 & lantha screen \\
\hline ZAK & 14 & 14 & 14 & lantha screen \\
\hline TNIK & 13 & 15 & 14 & lantha screen \\
\hline CLK3 & 14 & 14 & 14 & z-lyte \\
\hline AURKC (Aurora C) & 25 & 3 & 14 & z-lyte \\
\hline MAP4K3 (GLK) & 13 & 15 & 14 & lantha screen \\
\hline MAP3K5 (ASK1) & 3 & 24 & 14 & lantha screen \\
\hline PI4KB (PI4K beta) & 10 & 17 & 14 & adapta \\
\hline RIPK3 & 13 & 14 & 14 & lantha screen \\
\hline CDK16 (PCTK1)/cyclin Y & 14 & 13 & 14 & lantha screen \\
\hline AMPK (A1/B2/G1) & 13 & 14 & 14 & lantha screen \\
\hline MYO3A (MYO3 alpha) & 15 & 12 & 14 & lantha screen \\
\hline SBK1 & 13 & 13 & 13 & z-lyte \\
\hline MASTL & 13 & 13 & 13 & lantha screen \\
\hline ERBB4 (HER4) & 11 & 16 & 13 & z-lyte \\
\hline FGFR3 V555M & 14 & 13 & 13 & z-lyte \\
\hline MET D1228H & 13 & 14 & 13 & lantha screen \\
\hline MAPK10 (JNK3) & 13 & 13 & 13 & z-lyte \\
\hline PLK2 & 14 & 12 & 13 & z-lyte \\
\hline EGFR (ErbB1) d746-750 & 14 & 12 & 13 & lantha screen \\
\hline MLK4 & 9 & 16 & 13 & lantha screen \\
\hline MAPK3 (ERK1) & 15 & 11 & 13 & z-lyte \\
\hline STK3 (MST2) & 13 & 12 & 13 & z-lyte \\
\hline KIT Y823D & 11 & 14 & 13 & lantha screen \\
\hline SIK1 & 10 & 15 & 13 & lantha screen \\
\hline FGFR3 K650E & 12 & 13 & 13 & z-lyte \\
\hline IRAK4 & 11 & 14 & 12 & z-lyte \\
\hline AXL R499C & 14 & 11 & 12 & lantha screen \\
\hline CASK & 12 & 13 & 12 & lantha screen \\
\hline STK4 (MST1) & 13 & 12 & 12 & z-lyte \\
\hline CDK9/cyclin T1 & 10 & 14 & 12 & adapta \\
\hline NEK8 & 12 & 12 & 12 & lantha screen \\
\hline PKMYT1 & 13 & 11 & 12 & lantha screen \\
\hline INSR & 13 & 11 & 12 & z-lyte \\
\hline BRAF V599E & 10 & 14 & 12 & lantha screen \\
\hline IGF1R & 13 & 11 & 12 & z-lyte \\
\hline
\end{tabular}




\begin{tabular}{|c|c|c|c|c|}
\hline EPHB 1 & 12 & 12 & 12 & z-lyte \\
\hline PASK & 11 & 13 & 12 & z-lyte \\
\hline $\begin{array}{l}\text { PIK3CA/PIK3R3 (p110 } \\
\text { alpha/p55 gamma) }\end{array}$ & 13 & 11 & 12 & adapta \\
\hline PRKCB1 (PKC beta I) & 11 & 12 & 12 & z-lyte \\
\hline FGFR2 N549H & 14 & 9 & 12 & z-lyte \\
\hline PIP5K1B & 12 & 11 & 12 & adapta \\
\hline TAOK1 & 9 & 14 & 12 & lantha screen \\
\hline EGFR (ErbB1) G719C & 10 & 13 & 12 & z-lyte \\
\hline ЕРНА6 & 13 & 10 & 12 & lantha screen \\
\hline PTK2B (FAK2) & 11 & 12 & 11 & z-lyte \\
\hline MAP2K5 (MEK5) & 11 & 11 & 11 & lantha screen \\
\hline CLK1 & 11 & 11 & 11 & z-lyte \\
\hline CDK2/cyclin $\mathrm{O}$ & 14 & 8 & 11 & lantha screen \\
\hline KIT N822K & 12 & 11 & 11 & lantha screen \\
\hline RET V804M & 10 & 12 & 11 & lantha screen \\
\hline FLT3 ITD & 11 & 11 & 11 & lantha screen \\
\hline RAF1 (cRAF) Y340D Y341D & 12 & 10 & 11 & lantha screen \\
\hline FRK (PTK5) & 12 & 10 & 11 & z-lyte \\
\hline JAK1 & 10 & 12 & 11 & z-lyte \\
\hline ABL1 F317I & 9 & 12 & 11 & z-lyte \\
\hline PRKCD (PKC delta) & 10 & 11 & 10 & z-lyte \\
\hline $\begin{array}{l}\text { PIK3CB/PIK3R1 (p110 } \\
\text { beta/p85 alpha) }\end{array}$ & 10 & 10 & 10 & adapta \\
\hline CSK & 11 & 10 & 10 & z-lyte \\
\hline AMPK (A2/B2/G1) & 9 & 11 & 10 & lantha screen \\
\hline CDK14 (PFTK1)/cyclin Y & 11 & 9 & 10 & lantha screen \\
\hline EPHB2 & 11 & 10 & 10 & z-lyte \\
\hline KIT D820E & 8 & 12 & 10 & lantha screen \\
\hline AXL & 11 & 9 & 10 & z-lyte \\
\hline EPHA8 & 10 & 10 & 10 & z-lyte \\
\hline ULK2 & 10 & 10 & 10 & lantha screen \\
\hline DDR2 & 10 & 10 & 10 & lantha screen \\
\hline PI4K2B (PI4K2 beta) & 10 & 10 & 10 & adapta \\
\hline PIK3C2G (PI3K-C2 gamma) & 10 & 9 & 10 & adapta \\
\hline STK16 (PKL12) & 11 & 9 & 10 & lantha screen \\
\hline ERN1 & 12 & 7 & 10 & lantha screen \\
\hline SYK & 10 & 10 & 10 & z-lyte \\
\hline PHKG1 & 10 & 9 & 10 & z-lyte \\
\hline EGFR (ErbB1) G719S & 13 & 6 & 10 & z-lyte \\
\hline MERTK (cMER) & 9 & 10 & 10 & z-lyte \\
\hline
\end{tabular}




\begin{tabular}{|c|c|c|c|c|}
\hline GRK1 & 6 & 13 & 9 & lantha screen \\
\hline FLT4 (VEGFR3) & 9 & 10 & 9 & z-lyte \\
\hline ITK & 11 & 8 & 9 & z-lyte \\
\hline LIMK2 & 9 & 10 & 9 & lantha screen \\
\hline PTK6 (Brk) & 7 & 11 & 9 & z-lyte \\
\hline CSNK1A1L & 9 & 10 & 9 & z-lyte \\
\hline PDK1 & 9 & 9 & 9 & z-lyte \\
\hline $\begin{array}{l}\text { PIK3CA E545K/PIK3R1 (p110 } \\
\text { alpha E545K/p85 alpha) }\end{array}$ & 6 & 12 & 9 & adapta \\
\hline PIK3C2B (PI3K-C2 beta) & 11 & 7 & 9 & adapta \\
\hline ABL1 F317L & 8 & 9 & 9 & z-lyte \\
\hline FGFR3 & 16 & 2 & 9 & z-lyte \\
\hline TESK1 & 8 & 8 & 8 & lantha screen \\
\hline TNK1 & 7 & 9 & 8 & z-lyte \\
\hline $\begin{array}{l}\text { EGFR (ErbB1) T790M C797S } \\
\text { L858R }\end{array}$ & 8 & 8 & 8 & z-lyte \\
\hline BRAF & 6 & 10 & 8 & lantha screen \\
\hline HIPK2 & 8 & 8 & 8 & z-lyte \\
\hline EGFR (ErbB1) & 11 & 5 & 8 & z-lyte \\
\hline MAPK15 (ERK7) & 7 & 9 & 8 & lantha screen \\
\hline TLK1 & 9 & 7 & 8 & lantha screen \\
\hline FGFR4 & 6 & 9 & 8 & z-lyte \\
\hline AMPK (A2/B2/G2) & 8 & 7 & 8 & lantha screen \\
\hline SIK3 & 8 & 7 & 7 & lantha screen \\
\hline PI4K2A (PI4K2 alpha) & 5 & 10 & 7 & adapta \\
\hline MST1R (RON) & 7 & 7 & 7 & z-lyte \\
\hline PDGFRA D842V & 7 & 7 & 7 & z-lyte \\
\hline MAPK9 (JNK2) & 4 & 10 & 7 & z-lyte \\
\hline PHKG2 & 5 & 9 & 7 & z-lyte \\
\hline MAP3K2 (MEKK2) & 7 & 6 & 7 & lantha screen \\
\hline AMPK (A2/B2/G3) & 5 & 9 & 7 & z-lyte \\
\hline CDK1/cyclin B & 5 & 8 & 6 & z-lyte \\
\hline TBK1 & 8 & 5 & 6 & z-lyte \\
\hline EEF2K & 6 & 6 & 6 & z-lyte \\
\hline STK25 (YSK1) & 5 & 8 & 6 & z-lyte \\
\hline DAPK1 & 8 & 4 & 6 & adapta \\
\hline SRPK2 & 7 & 5 & 6 & z-lyte \\
\hline TEK (TIE2) R849W & 2 & 10 & 6 & lantha screen \\
\hline CDK9/cyclin $\mathrm{K}$ & 5 & 7 & 6 & lantha screen \\
\hline EGFR (ErbB1) C797S & 4 & 8 & 6 & z-lyte \\
\hline STK33 & 6 & 6 & 6 & lantha screen \\
\hline
\end{tabular}




\begin{tabular}{|c|c|c|c|c|}
\hline CAMKK1 (CAMKKA) & 6 & 6 & 6 & lantha screen \\
\hline DCAMKL2 (DCK2) & 4 & 7 & 6 & z-lyte \\
\hline CSNK1A1 (CK1 alpha 1) & 4 & 7 & 6 & z-lyte \\
\hline CDK17/cyclin Y & 5 & 7 & 6 & z-lyte \\
\hline MAPK13 (p38 delta) & 5 & 6 & 6 & z-lyte \\
\hline CSNK2A1 (CK2 alpha 1) & 6 & 5 & 6 & z-lyte \\
\hline DYRK1A & 6 & 5 & 6 & z-lyte \\
\hline MAPK11 (p38 beta) & 6 & 5 & 5 & z-lyte \\
\hline HUNK & 6 & 5 & 5 & lantha screen \\
\hline TAOK2 (TAO1) & 5 & 5 & 5 & z-lyte \\
\hline KIT T670E & 5 & 5 & 5 & lantha screen \\
\hline ICK & 4 & 6 & 5 & lantha screen \\
\hline JAK2 JH1 JH2 V617F & 6 & 4 & 5 & z-lyte \\
\hline PDGFRB (PDGFR beta) & 5 & 5 & 5 & z-lyte \\
\hline TESK2 & 5 & 5 & 5 & lantha screen \\
\hline EPHA5 & 5 & 5 & 5 & z-lyte \\
\hline PIK3C2A (PI3K-C2 alpha) & 2 & 7 & 5 & adapta \\
\hline PDGFRA (PDGFR alpha) & 6 & 3 & 5 & z-lyte \\
\hline $\begin{array}{l}\text { PIK3CB/PIK3R2 (p110 } \\
\text { beta/p85 beta) }\end{array}$ & 4 & 5 & 4 & adapta \\
\hline KIT V654A & 3 & 5 & 4 & lantha screen \\
\hline GRK7 & 1 & 7 & 4 & z-lyte \\
\hline FRAP1 (mTOR) & 6 & 2 & 4 & z-lyte \\
\hline KIT T670I & 5 & 2 & 4 & z-lyte \\
\hline NEK4 & 1 & 6 & 3 & z-lyte \\
\hline WNK3 & 2 & 5 & 3 & lantha screen \\
\hline PRKCI (PKC iota) & -1 & 7 & 3 & z-lyte \\
\hline CDC42 BPA (MRCKA) & 1 & 5 & 3 & z-lyte \\
\hline BRSK2 & 3 & 3 & 3 & lantha screen \\
\hline PI4KA (PI4K alpha) & 9 & -3 & 3 & adapta \\
\hline SRPK1 & 3 & 3 & 3 & z-lyte \\
\hline ERBB2 (HER2) & 2 & 3 & 3 & z-lyte \\
\hline DMPK & 2 & 3 & 3 & lantha screen \\
\hline KIT V559D V654A & 2 & 3 & 2 & z-lyte \\
\hline FYN A & -1 & 6 & 2 & lantha screen \\
\hline ZAP70 & 1 & 4 & 2 & z-lyte \\
\hline PDGFRA V561D & -6 & 10 & 2 & z-lyte \\
\hline INSRR (IRR) & 1 & 3 & 2 & z-lyte \\
\hline PAK1 & 2 & 2 & 2 & z-lyte \\
\hline CDC42 BPB (MRCKB) & 3 & 2 & 2 & z-lyte \\
\hline PIK3C3 (hVPS34) & 3 & 2 & 2 & adapta \\
\hline
\end{tabular}




\begin{tabular}{|c|c|c|c|c|}
\hline PIP4K2A & 5 & -1 & 2 & adapta \\
\hline KIT V560G & 2 & 2 & 2 & z-lyte \\
\hline PDGFRA T674I & 3 & 1 & 2 & z-lyte \\
\hline TEK (TIE2) Y1108F & -3 & 7 & 2 & lantha screen \\
\hline DDR1 & 1 & 3 & 2 & lantha screen \\
\hline DYRK4 & 2 & 1 & 2 & z-lyte \\
\hline NEK9 & 3 & 0 & 2 & z-lyte \\
\hline KIT V559D & 1 & 1 & 1 & z-lyte \\
\hline KIT & 2 & 1 & 1 & z-lyte \\
\hline CSNK2A2 (CK2 alpha 2) & 3 & -1 & 1 & z-lyte \\
\hline FLT1 (VEGFR1) & 0 & 2 & 1 & z-lyte \\
\hline EGFR (ErbB1) L858R & 1 & 2 & 1 & z-lyte \\
\hline DAPK3 (ZIPK) & 0 & 3 & 1 & z-lyte \\
\hline NIM1K & 0 & 2 & 1 & z-lyte \\
\hline CDC42 BPG (MRCKG) & 2 & 0 & 1 & z-lyte \\
\hline ACVR1B (ALK4) & -2 & 4 & 1 & z-lyte \\
\hline HIPK3 (YAK1) & 0 & 1 & 1 & z-lyte \\
\hline EGFR (ErbB1) L861Q & 1 & 0 & 1 & z-lyte \\
\hline CDK4/cyclin D3 & 0 & 2 & 1 & adapta \\
\hline WNK1 & 1 & 0 & 1 & lantha screen \\
\hline HIPK1 (Myak) & 1 & 0 & 0 & z-lyte \\
\hline PDK1 Direct & 0 & 0 & 0 & z-lyte \\
\hline MET (cMet) Y1235D & -1 & 1 & 0 & z-lyte \\
\hline CDK4/cyclin D1 & -2 & 2 & 0 & adapta \\
\hline MAP3K3 (MEKK3) & 3 & -4 & 0 & lantha scree \\
\hline MAPK14 (p38 alpha) Direct & -2 & 1 & -1 & z-lyte \\
\hline CSF1R (FMS) & -1 & -1 & -1 & z-lyte \\
\hline CDKL5 & -1 & -1 & -1 & z-lyte \\
\hline MKNK1 (MNK1) & -1 & -2 & -2 & z-lyte \\
\hline DNA-PK & -7 & 3 & -2 & z-lyte \\
\hline PIM1 & -3 & -1 & -2 & z-lyte \\
\hline CDK18/cyclin Y & -2 & -2 & -2 & z-lyte \\
\hline PAK3 & 4 & -9 & -2 & z-lyte \\
\hline LTK (TYK1) & -3 & -2 & -2 & z-lyte \\
\hline PIP5K1C & -2 & -2 & -2 & adapta \\
\hline EPHA3 & -5 & -1 & -3 & lantha screen \\
\hline MAPK12 (p38 gamma) & -4 & -3 & -3 & z-lyte \\
\hline PIK3CG (p110 gamma) & -8 & 1 & -4 & adapta \\
\hline DCAMKL1 (DCLK1) & -5 & -3 & -4 & z-lyte \\
\hline CDK6/cyclin D1 & 0 & -10 & -5 & adapta \\
\hline PKN1 (PRK1) & -7 & -4 & -5 & z-lyte \\
\hline
\end{tabular}




\begin{tabular}{lllll} 
PIP5K1A & -4 & -7 & -5 & adapta \\
RIPK2 & -7 & -6 & -6 & lantha screen \\
PIM3 & -8 & -6 & -7 & z-lyte \\
PRKCE (PKC epsilon) & -7 & -7 & -7 & z-lyte \\
LIMK1 & -4 & -10 & -7 & lantha screen \\
WNK2 & -8 & -7 & -7 & lantha screen \\
CDK9 (Inactive) & -7 & -8 & -8 & lantha screen \\
IKBKE (IKK epsilon) & -11 & -9 & -10 & z-lyte \\
PRKCZ (PKC zeta) & -9 & -11 & -10 & z-lyte \\
PIM2 & -12 & -12 & -12 & z-lyte \\
GRK5 & -14 & -13 & -13 & z-lyte \\
MATK (HYL) & -23 & -15 & -19 & z-lyte \\
SRC N1 & -22 & -22 & -22 & z-lyte \\
SRC & -25 & -26 & -26 & z-lyte \\
IKBKB (IKK beta) & -99 & -95 & -97 & z-lyte \\
\hline
\end{tabular}

